\title{
Investigation of structural properties of organic thin films for solar cell and transistor applications
}

\author{
Ronak Rahimi \\ West Virginia University
}

Follow this and additional works at: https://researchrepository.wvu.edu/etd

\section{Recommended Citation}

Rahimi, Ronak, "Investigation of structural properties of organic thin films for solar cell and transistor applications" (2012). Graduate Theses, Dissertations, and Problem Reports. 4911.

https://researchrepository.wvu.edu/etd/4911

This Dissertation is protected by copyright and/or related rights. It has been brought to you by the The Research Repository @ WVU with permission from the rights-holder(s). You are free to use this Dissertation in any way that is permitted by the copyright and related rights legislation that applies to your use. For other uses you must obtain permission from the rights-holder(s) directly, unless additional rights are indicated by a Creative Commons license in the record and/ or on the work itself. This Dissertation has been accepted for inclusion in WVU Graduate Theses, Dissertations, and Problem Reports collection by an authorized administrator of The Research Repository @ WVU.

For more information, please contact researchrepository@mail.wvu.edu. 


\title{
INVESTIGATION OF STRUCTURAL PROPERTIES OF ORGANIC THIN FILMS FOR SOLAR CELL AND TRANSISTOR APPLICATIONS
}

\author{
RONAK RAHIMI
}

\begin{abstract}
Dissertation submitted to the
at West Virginia University

Doctor of Philosophy

in

Electrical Engineering

Dimitris Korakakis, Ph.D., Chair

Jeremy Dawson, Ph.D.

Parviz Famouri, Ph.D.

David W. Graham, Ph.D.

Charter D. Stinespring, Ph.D.
\end{abstract}

Benjamin M. Statler College of Engineering and Mineral Resources

in partial fulfillment of the requirements for the degree of

Lane Department of Computer Science and Electrical Engineering

Morgantown, West Virginia

2012

Keywords: Thin films, Organic solar cells, Organic transistors Copyright (c) 2012 Ronak Rahimi 


\title{
Investigation of structural properties of organic thin films for solar cell and transistor applications
}

\author{
Ronak Rahimi
}

\section{ABSTRACT}

For the past several decades, organic materials including polymers, oligomers and small molecules have been of great interest for their various applications in the electronics and the semiconductor industry. The most appealing advantages of organic materials compared to their inorganic counterparts are their compatibility with flexible substrates and amenability to lowtemperature and low-cost fabrication processes such as evaporation, spin-coating and printing. Moreover, the ability to be utilized in fabrication of lightweight and large-area devices is among other reasons for popularity of organic materials. A large number of studies have reported on various aspects of the development and optimization of organic electronics such as organic light emitting diodes (OLEDs), solar cells (OSCs) and thin film transistors (OTFTs). Although significant progress has been made during this period, some of the intrinsic electrical properties of organic materials such as low carrier mobility have continued to hinder the full development and maturation of the organic electronics industry. In order to manufacture organic electronic devices with high performance, more detailed studies of the structure and the morphology of the organic materials as well as the underlying physical charge transport mechanisms should be performed. Additionally, growth, deposition and assembly processes need to be established and optimized for the new organic semiconductor technology. 
This work aims to advance the understanding of the effect of the structural properties of organic thin films on the charge carrier transport within the organic thin films as well as the charge carrier injection between the organic layers and the organic-inorganic materials such as metal or dielectric layers. Charge carrier transport mechanisms between different layers are crucial factors in determining the efficiency of organic electronic devices. These parameters rely largely on the molecular structure, morphology and ordering of the organic thin films. In order to investigate these intrinsic properties, several organic thin films were prepared using vacuum thermal evaporation method. Their morphology and structural properties were studied by the combination of various techniques including atomic force microscopy, X-ray reflectivity, spectroscopic ellipsometry and transmittance measurements. Based on the produced organic thin films, organic semiconductor devices such as OTFTs and OSCs were fabricated and their electrical and optical properties were characterized. Moreover, the effect of morphology and structure of the organic thin films on the organic device performance was studied. Ambipolar thin film transistors based on pentacene and PTCDI-C8 as the active layer and lithium fluoride (LiF) as the gate dielectric layer were fabricated and characterized. Conduction behaviors of these devices were modeled using Fowler-Nordheim (FN) tunneling theory. The results of this study suggest that the charge transport in OTFTs correlate not only with the organic semiconductor film structure, but also with the dielectric-semiconductor interfacial effects. Moreover, bilayer heterojunction OSCs based on CuPc/PTCDI-C8 as the donor/acceptor layers were fabricated and their electrical and optical properties were characterized. The effects of the active layers' structures and morphologies as well as the buffer layers' thickness variation on the device performance were studied. The results of this study emphasized the importance of the thin film structural properties on the device performance. 


\section{ACKNOWLEDGMENTS}

I would like to express my deepest appreciation and gratitude to my supervisor, Dr. Dimitris Korakakis, for giving me the opportunity and guidance to work in the nanoelectronics field. His knowledge and encouragement have been of utmost value throughout the course of my research.

I also wish to express my sincere thanks to the members of my committee, Dr. Jeremy Dawson, Dr. Parviz Famouri, Dr. David W. Graham and Dr. Charter D. Stinespring whose understanding and constructive comments have been most valuable throughout this work.

Most importantly, I'd like to thank my family for their loving support and constant encouragement. I would not have made it this far without their support.

Lastly, I would like to thank all of my colleagues in the Materials Growth and Characterization Lab. Special recognition is warranted to Alex Roberts, Vishl Narang and Vamsi Kumbham for their co-operation and support. 
ACKNOWLEDGMENTS

2.1. POLYMERS

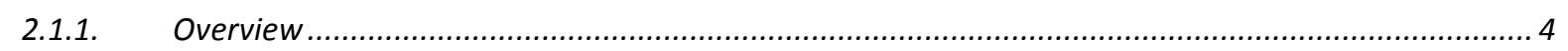

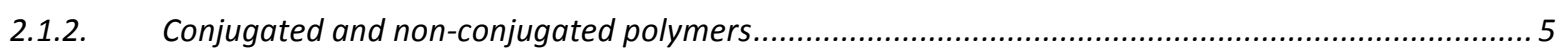

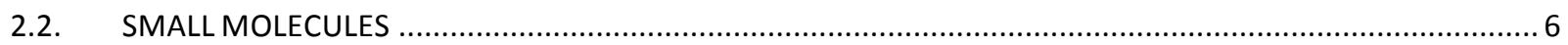

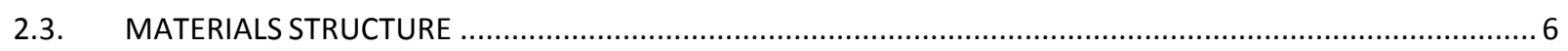

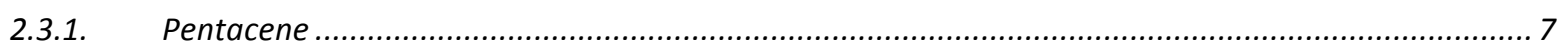

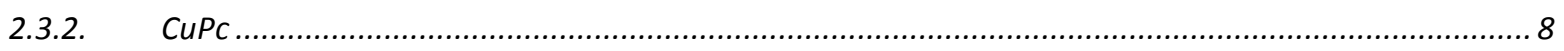

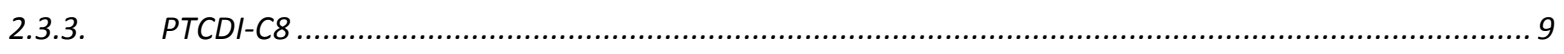

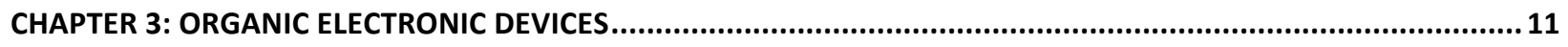

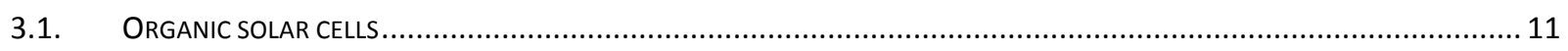

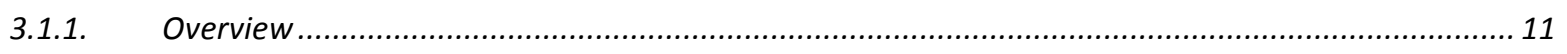

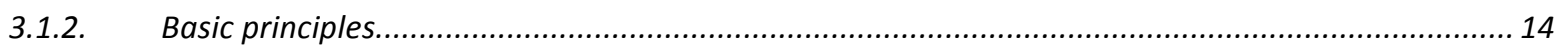

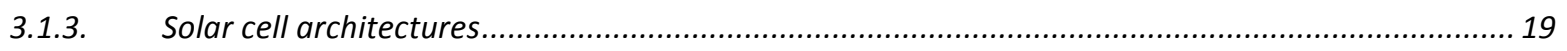

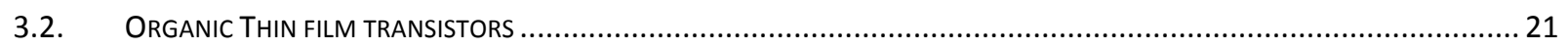

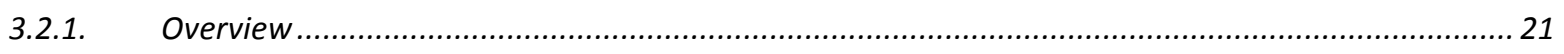

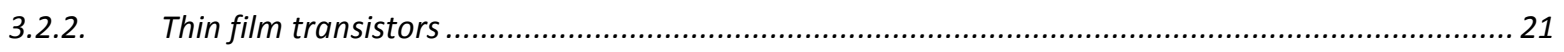

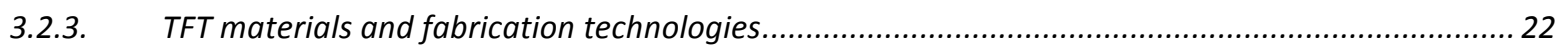

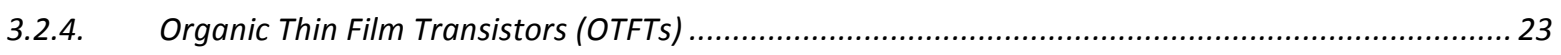

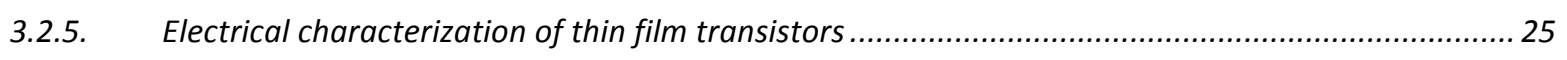

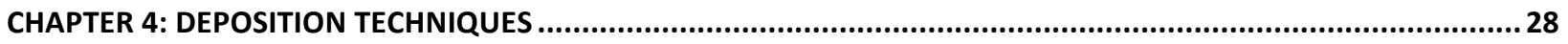

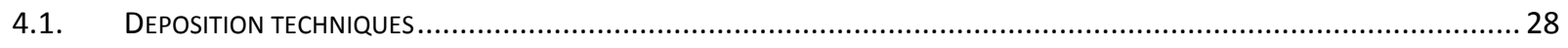

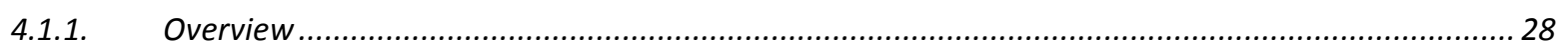

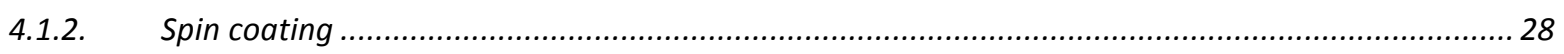

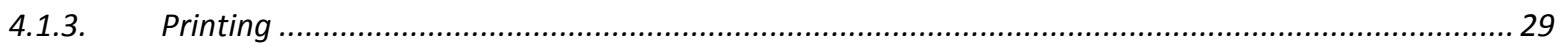

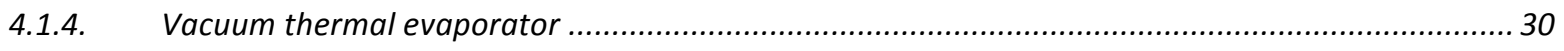

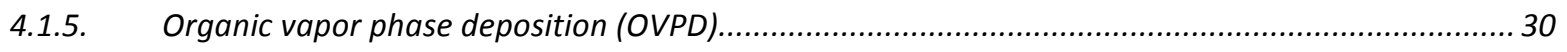

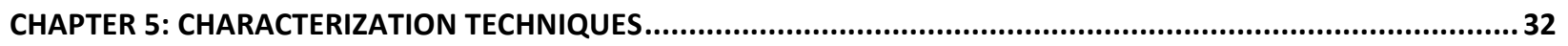




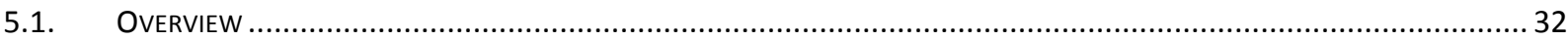

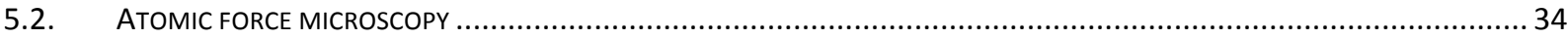

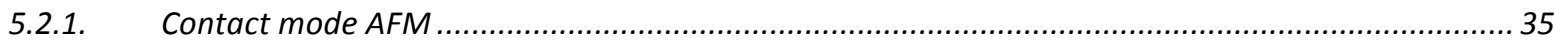

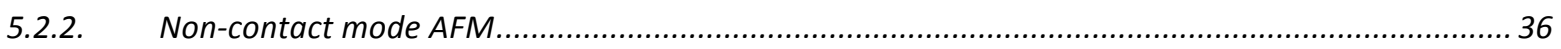

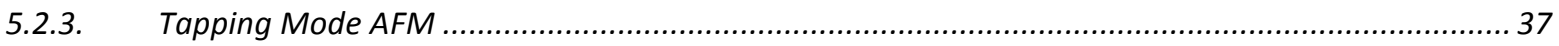

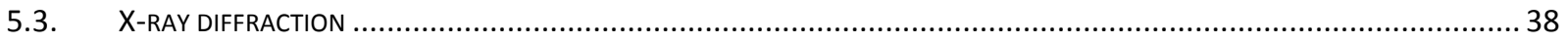

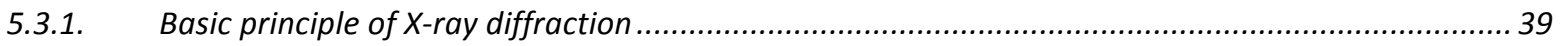

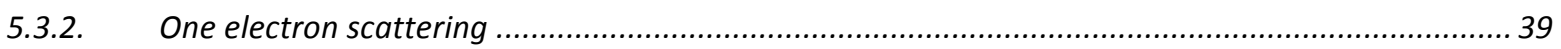

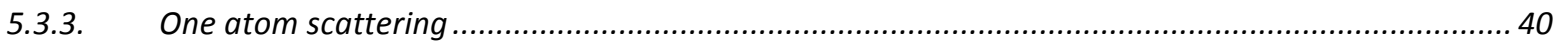

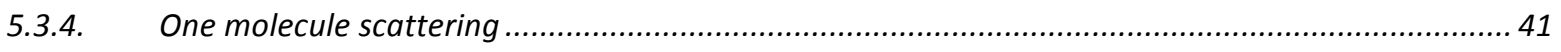

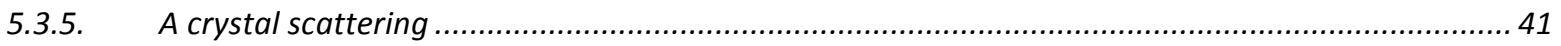

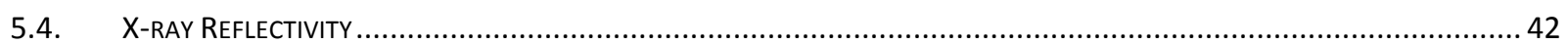

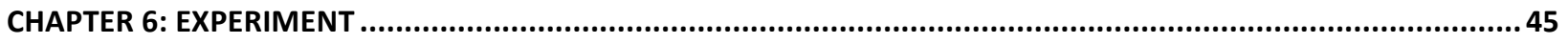

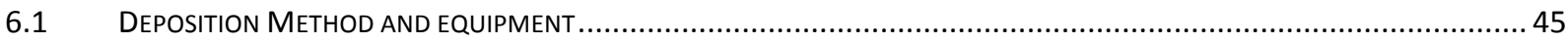

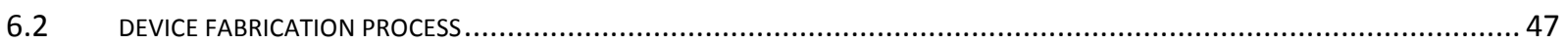

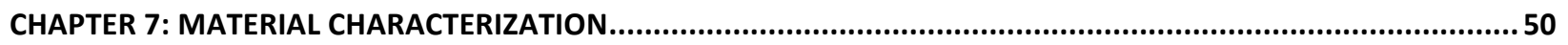

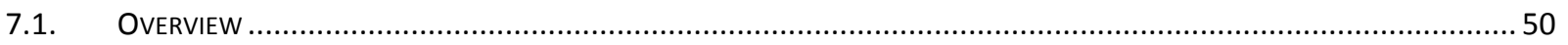

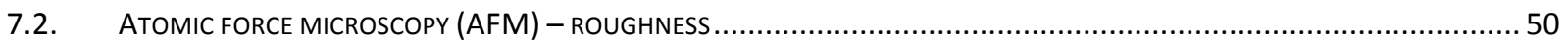

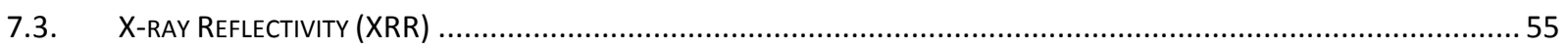

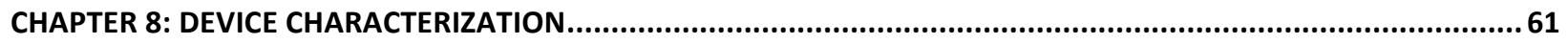

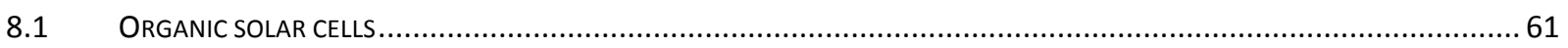

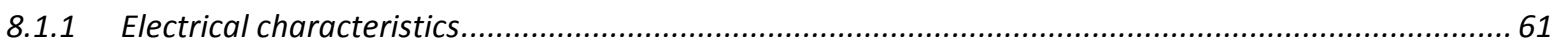

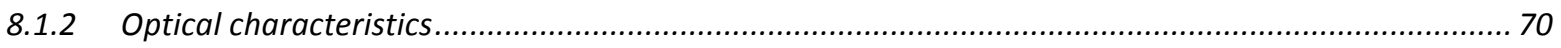

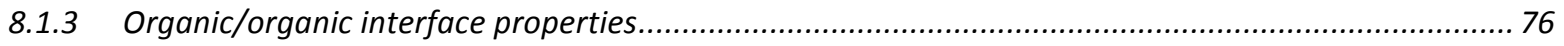

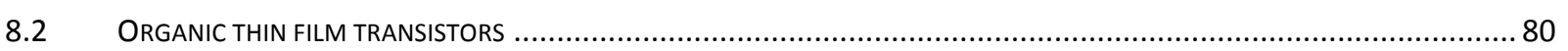

8.2.1 Electrical characteristics - ambipolar behavior ......................................................................... 80

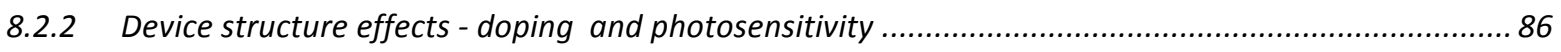

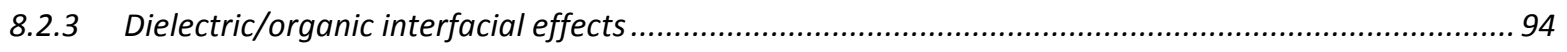

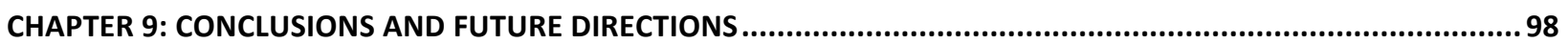

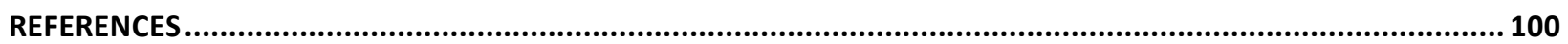

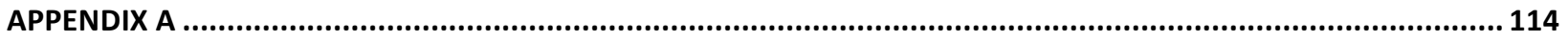

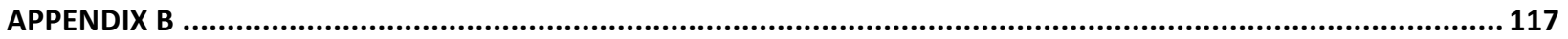




\section{LIST OF FIGURES}

FiguRE 2.1 - CheMiCAL REPRESENTATION OF PENTACENE MOLECULE (LEFT), PENTACENE THIN FILM WITH LAYERED STRUCTURE (MIDDLE)

[15], SCHEMATIC OF A UNIT CELL AND HERRINGBONE PACKING OF PENTACENE MOLECULES (RIGHT) [12].

Figure 2.2 - (A) Chemical RePresentation of the isoindole subunits, (B) Copper Phthalocyanine (CuPC) MOLECULE, MOLECULAR ARRANGEMENT IN CUPC THIN FILMS OF THE, (C) A-FORM, AND (D) B- FORM [24] ....................................... 9

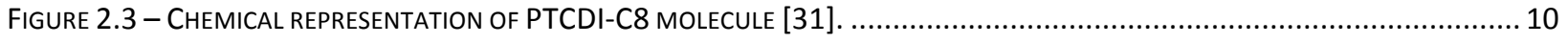

FIGURE 3.1 - A SUMMARY OF THE DIFFERENT LOSS PROCESSES IN A TYPICAL SOLAR CELL [34]............................................ 11

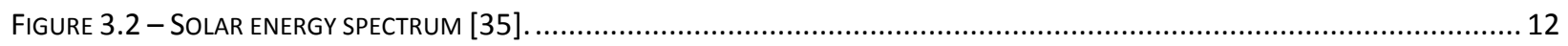

FIGURE 3.3 - AN OVERVIEW OF THE POWER CONVERSION EFFICIENCY OF ORGANIC SOLAR CELLS PROVIDED BY THE NATIONAL RENEWABLE

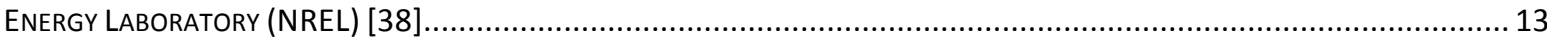

FIGURE 3.4 - PHOTOINDUCED CHARGE GENERATION AND COLLECTION PROCESSES FOR A TYPICAL DONOR/ACCEPTOR BILAYER SOLAR CELL.

FIGURE 3.5 - EQUIVALENT CIRCUIT FOR A SOLAR CELL WITH SHUNT AND SERIES RESISTORS............................................... 15

FIGURE 3.6 - TYPICAL I-V PLOT OF A SOLAR SELL UNDER DARK AND LIGHT CONDITIONS. ................................................ 17

FIGURE 3.7 - MAXIMUM POWER FOR AN I-V SWEEP OF A SOLAR CELL. ....................................................................... 18

FIGURE 3.8 - EXAMPLES OF DIFFERENT POSSIBLE SOLAR CELL ARCHITECTURES........................................................... 19

FIGURE 3.9 - BASIC THIN FILM TRANSISTORS CONFIGURATION, (A) TOP-GATED TOP CONTACTS CONFIGURATION, (B) TOP-GATED BOTTOM CONTACTS CONFIGURATION, (C) BOTTOM-GATED TOP CONTACTS CONFIGURATION, (D) BOTTOM-GATED BOTTOM CONTACTS

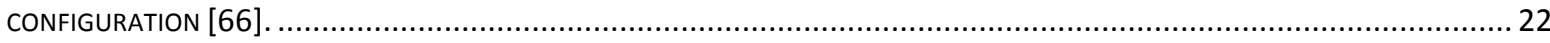

FIGURE 3.10 - TYPICAL OTFT STRUCTURE WITH SIO2 AS THE GATE INSULATING LAYER [67] ............................................24

FIGURE 3.11 - TYPICAL OUTPUT CHARACTERISTICS OF A N-TYPE THIN FILM TRANSISTOR......................................................26

FIGURE 4.1 - SCHEMATIC OF ORGANIC FILM FORMATION BY INKJET PRINTING AND THE STRUCTURE OF THE FABRICATED DEVICE

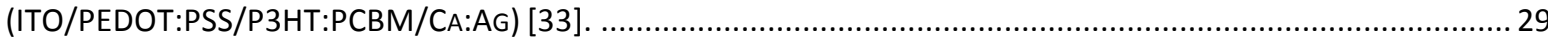

FIGURE 4.2 - SCHEMATIC OF A VACUUM THERMAL EVAPORATOR (VTE) SYSTEM. ......................................................... 30

FIGURE 4.3 - SCHEMATIC OF AN ORGANIC VAPOR PHASE DEPOSITION (OVPD) SYSTEM................................................ 31

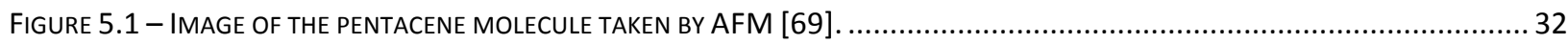

FIGURE 5.2 - SCHEMATIC EVOLUTIONS OF THIN-FILM POLYMORPHS OF PENTACENE FILM WITH INCREASING THICKNESS [12].............. 33

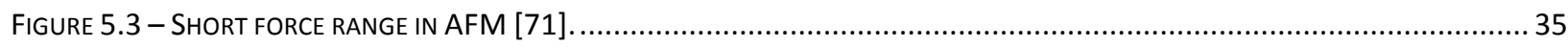

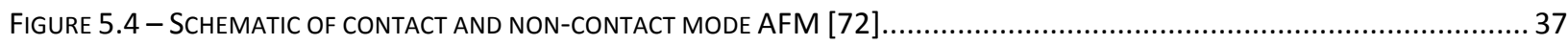

FIGURE 5.5 - THE X-RAY SCATTERING FROM (A) AN ELECTRON, (B) ONE ATOM AND (C) A CRYSTAL. ........................................ 41

FIGURE 5.6 - SCHEMATIC OF THE REFLECTION FOR A FILM WITH A MULTILAYERED STRUCTURE. ............................................ 43

FIGURE 5.7 - REFLECTIVITY PATTERNS AS A FUNCTION OF MOMENTUM TRANSFER FOR (A) A SMOOTH VACUUM/MEDIUM INTERFACE, (B) A FILM WITH TWO INTERFACES, (C) A FILM WITH A PERIODIC ELECTRON DENSITY AND (D) A FILM SAME AS PREVIOUS FILM BUT

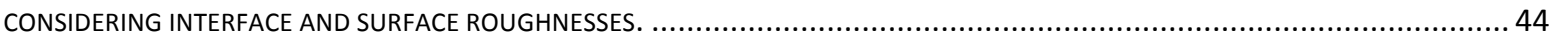

FiguRE 6.1 - THE ACADEMIC SERIES RESEARCH EVAPORATOR FROM TROVATO MFG., INC WITH THE ATTACHED GLOVE BOX. ............... 45

FIGURE 6.2 - SCHEMATIC OF DIFFERENT PARTS OF THE THERMAL EVAPORATOR SYSTEM.................................................46

FIGURE 6.3 - THE SUBSTRATE CLEANING PROCESS USED IN THIS WORK..................................................................... 47

FIGURE 6.4 - THE FABRICATION PROCESS USED FOR (A) ORGANIC SOLAR CELLS AND (B) ORGANIC TRANSISTORS...............................48

FIGURE 6.5 - SCHEMATIC DIAGRAM OF THE FABRICATED (A) SOLAR CELLS WITH THE FOLLOWING STRUCTURE: (ITO/MOO $3 / \mathrm{CUPC} /$ PTCDIC8/ALQ $3 / A L)$ AND (B) TRANSISTORS WITH THE TOP CONTACT GEOMETRY.

FIGURE 7.1 - AFM IMAGES OF (A) ITO SUBSTRATES ANNEALED AT 500 ㅇ IN NITROGEN FOR 10 MIN, AND (B) SILICON SUBSTRATES WITH NATIVE SILICON DIOXIDE. 51 
FIGURE 7.2 - AFM IMAGES OF THIN FILM OF 39 NM PTCDI-C8 DEPOSITED ON ITO SUBSTRATE AT ROOM TEMPERATURE SHOWING $\Sigma$ $5.3 \pm 0.25 \mathrm{NM}$. THE ITO SUBSTRATES WERE ANNEALED AT 500 ㅇ IN NITROGEN FOR 10 MIN BEFORE FILM DEPOSITION............5 52 52

FIGURE 7.3 - AFM IMAGES OF THIN FILM OF 27 NM PTCDI-C8 DEPOSITED ON SILICON SUBSTRATE AT ROOM TEMPERATURE. THESE FILMS DISPLAY VERY SMOOTH SURFACES WITH $\Sigma \sim 1.04 \pm 0.04 \mathrm{NM}$. 52

FIGURE 7.4 - AFM IMAGES OF 20, 27, 33 AND 45 NM THICK PTCDI-C8 FILMS DEPOSITED ON SILICON SUBSTRATE AT ROOM TEMPERATURE. 53

FIGURE 7.5 - PLOT OF FILM ROUGHNESS AS A FUNCTION OF THICKNESS FOR PTCDI-C8 FILMS DEPOSITED ON SILICON SUBSTRATES. ...... 54

FIGURE 7.6 - X-RAY REFLECTIVITY CURVES FOR DIFFERENT THICKNESSES OF PTCDI-C8 FILMS................................................ 55

FIGURES 7.7 - MEASURED AND SIMULATED REFLECTIVITY DATA FOR PTCDI-C8 FILM WITH 27 NM THICKNESS. ............................. 56

FIGURE 7.8 - COMPARING THE MEASURED AND CALCULATED ROUGHNESS FROM AFM AND X-RAY REFLECTIVITY MEASUREMENTS. ....... 57

FIGURE 7.9 - COMPARING THE MEASURED AND CALCULATED THICKNESS FROM ELLIPSOMETRY AND X-RAY REFLECTIVITY MEASUREMENTS.

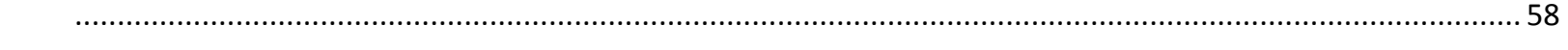

FIGURE 7.10 - CALCULATED INTERLAYER SPACING FOR PTCDI-C8 FILMS WITH DIFFERENT THICKNESSES................................... 59

FIGURE 7.11 - THE DOMAIN SIZE CALCULATED FOR THE DIFFERENT THICKNESSES OF PTCDI-C8 FILMS. .....................................60

FIGURE 8.1 - J-V DATA FOR DEVICES WITH DIFFERENT ALQ 3 THICKNESSES RANGING FORM 0 TO 15 NM.....................................6. 61

FIGURE 8.2 - OPEN CIRCUIT VOLTAGE ( $V_{\text {OC }}$ ) AND SHORT CIRCUIT CURRENT ( $\mathrm{J}_{S C}$ ) FOR DEVICES WITH DIFFERENT ETL THICKNESS IN THE

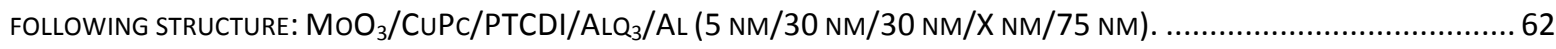

FIGURE 8.3 - OPEN CIRCUIT VOLTAGE $\left(V_{\text {OC }}\right.$ ) AND SHORT CIRCUIT CURRENT (J $\mathrm{J}_{S C}$ FOR DEVICES WITH DIFFERENT HTL THICKNESS IN THE FOLLOWING STRUCTURE: $\mathrm{MOO}_{3} / \mathrm{CUPC} / \mathrm{PTCDI} / \mathrm{ALQ}_{3} / \mathrm{AL}$ (X NM/30 NM/30 NM/7.5 NM/75 NM)

FIGURE 8.4 - J-V DATA FOR DEVICES WITH DIFFERENT ACTIVE LAYERS THICKNESSES IN THE FOLLOWING STRUCTURE: MOO 3 /CUPC/PTCDI$\mathrm{C} 8 / \mathrm{ALQ}_{3} / \mathrm{AL}$ (7.5 NM/X NM/Y NM/7.5 NM/75 NM)

FIGURE 8.5 - OPEN CIRCUIT VOLTAGE $\left(\mathrm{V}_{\text {OC }}\right.$ ) AND SHORT CIRCUIT CURRENT (J $\mathrm{J}_{S C}$ ) FOR DEVICES WITH DIFFERENT ACTIVE LAYERS THICKNESS IN

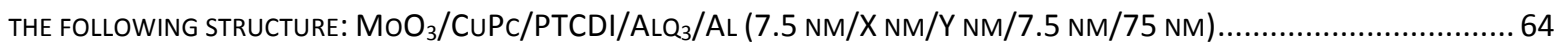

FIGURE 8.6 - CURRENT DENSITY OF DIFFERENT DEVICES AS A FUNCTION OF APPLIED BIAS UNDER LIGHT ILLUMINATION FOR VARIOUS ALQ 3 THICKNESSES. .. 65

FIGURE 8.7 - OPEN CIRCUIT VOLTAGE $\left(\mathrm{V}_{\text {OC }}\right)$, SHORT CIRCUIT CURRENT $\left(\mathrm{J}_{S C}\right)$, FILL FACTOR (FF), EFFICIENCY (H) AND PMAXFOR DEVICES WITH DIFFERENT ALQ 3 THICKNESS 67

FIGURE 8.8 - J-V CURVES OF DEVICES WITH 20, 25, 30 AND 35 NM ALQ 3 THICKNESSES IN DARK AND UNDER ILLUMINATION.............. 69

FIGURE 8.9 - SCHEMATIC ENERGY DIAGRAM OF CUPC/PTCDI-C8 BILAYER SOLAR CELLS SHOWING THE EFFECT OF METAL-INDUCES DEFECT

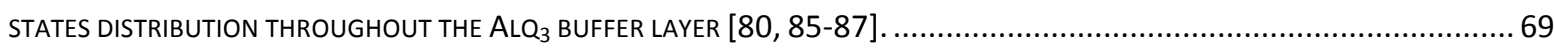

FIGURE 8.10 - SIMULATED AND MEASURED REFRACTIVE INDICES AND EXTINCTION COEFFICIENTS FOR $\mathrm{MOO}_{3} \ldots . . . . . . . . . . . . . . . . . . . . . . . . .71$

FIGURE 8.11 - SIMULATED REFLECTIVITY FOR SAMPLES WITH (A) DIFFERENT ITO THICKNESSES FROM 0 TO 300 NM, (B) 170 NM ITO AND DIFFERENT $\mathrm{MOO}_{3}$ THICKNESSES FROM 0 TO $40 \mathrm{NM}$, (C) $170 \mathrm{NM}$ ITO, 20 NM MOO 3 AND DIFFERENT CUPC THICKNESSES FROM 0 TO 70 NM, (D) 170 NM ITO, 20 NM $\mathrm{MOO}_{3}$, 30 NM CUPC AND DIFFERENT PTCDI-C8 THICKNESSES FROM 0 TO 70 NM.......... 72

FIGURE 8.12 - SIMULATED REFLECTIVITY FOR THE STRUCTURES WITH 170 NM ITO, 20 NM MoO 30 NM CUPC, 30 NM PTCDI-C8 AND DIFFERENT $\mathrm{ALQ}_{3}$ THICKNESSES FROM 0 TO $40 \mathrm{NM}$ 73

FIGURE 8.13 - SIMULATED AND EXPERIMENTAL REFLECTIVITY FOR THE FOLLOWING STRUCTURE: ITO (170NM) / MOO $_{3}$ (30NM) /CUPC (40NM).

FIGURE 8.14 - SIMULATED AND EXPERIMENTAL REFLECTIVITY FOR THE FOLLOWING STRUCTURE: ITO (170NM)/MOO 3 (20NM)/CUPC

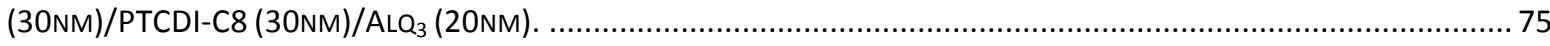

FIGURE 8.15 - SIMULATED AND EXPERIMENTAL XRR DATA FOR (A) BILAYER AND (B) LAYERED CUPC/PTCDI-C8 FILMS.................... 77

FIGURE 8.16 - SIMULATED AND EXPERIMENTAL XRR DATA FOR CUPC/PTCDI-C8 CO-DEPOSITED FILM......................................78

FIGURE 8.17 - J-V RESULTS OF OSCS WITH DIFFERENT ACTIVE LAYERS STRUCTURES. ........................................................ 79 


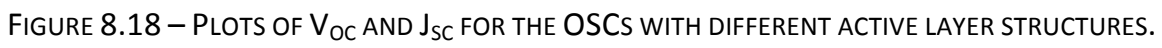

FIGURE 8.19 - PLOT OF DRAIN-SOURCE CURRENT (IDS) VS. DRAIN-SOURCE VOLTAGE (VDS) FOR DIFFERENT (A) POSITIVE AND (B) NEGATIVE GATE VOLTAGES IN THE TOP-CONTACTED PENTACENE-LIF AMBIPOLAR THIN FILM TRANSISTOR.....

FIGURE 8.20 - THE CORRESPONDING FOWLER-NORDHEIM (LN (I/E2) VS. 1/E) PLOTS FOR (A) POSITIVE, AND (B) NEGATIVE GATE VOLTAGES. THE INSETS SHOW THE LEAST-SQUARES ERROR FIT IN THE TUNNELING REGION FOR GATE VOLTAGES AT $\pm 15 \mathrm{~V}$.

FIGURE 8.21 - PLOT OF THE SOURCE-DRAIN VOLTAGE, AT WHICH TRANSITION FROM HOPPING TO TUNNELING OCCURS, VS (A) NEGATIVE AND (B) POSITIVE GATE VoltAges. THE GATE-VOLTAGE DEPENDENCE OF THE B AND $\Phi$ IN FOWLER-NORDHEIM LAW CALCULATED FROM THE SLOPE OF THE TUNNELING REGIONS IN FN PLOTS FOR (C) NEGATIVE AND (D) POSITIVE GATE VOLTAGES.

FiguRE 8.22 - SOURCE-DRAIN CURRENT-VOLTAGE CHARACTERISTICS OF THE OTFT WITH (A) PTCDI-C8 AND (B) PENTACENE AS THE

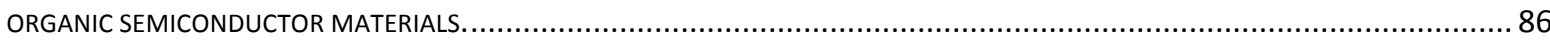

FIGURE 8.23 - RAMAN SPECTRUM WITH THE FITTED PEAKS FOR PENTACENE FILMS. THE INSETS SHOW THE EFFECT OF GATE BIAS ON THE RAMAN PEAKS.

FIGURE 8.24 - SOURCE-DRAIN I-V CHARACTERISTICS OF THE OTFT WITHOUT $\mathrm{V}_{2} \mathrm{O}_{5}$ LAYER BETWEEN LIF AND PENTACENE WITH DIFFERENT THICKNESS OF PENTACENE (65NM, 100NM, 135NM) AT VG $=-20 \mathrm{~V}$

FIGURE 8.25 - SOURCE-DRAIN CURRENT AS A FUNCTION OF PENTACENE THICKNESS FOR TWO DIFFERENT SOURCE-DRAIN VOLTAGES (10 V AND $25 \mathrm{~V}$ ) FOR OTFTS WITHOUT $\mathrm{V}_{2} \mathrm{O}_{5}$ LAYER BETWEEN LIF AND PENTACENE.

FIGURE 8.27 - SOURCE-DRAIN CURRENT-VOLTAGE CHARACTERISTICS OF THE TRANSISTORS WITH $\mathrm{V}_{2} \mathrm{O}_{5}$ LAYER BETWEEN LIF AND PENTACENE WITH DIFFERENT THICKNESSES OF PENTACENE AT VG = -20V. DRAIN CURRENT DROPS SIGNIFICANTLY COMPARED TO THE CURRENT FOR THE TRANSISTORS WITHOUT THIS LAYER. .90

FIGURE 8.26 - SOURCE-DRAIN CURRENT-VOLTAGE CHARACTERISTICS OF THE TRANSISTORS WITH AND WITHOUT $\mathrm{V}_{2} \mathrm{O}_{5}$ LAYER BETWEEN LIF AND PENTACENE WITH DIFFERENT THICKNESSES OF PENTACENE AT VG $=-20 \mathrm{~V}$.

FIGURE 8.28 - THE EFFECT OF LIGHT ON THE OUTPUT CHARACTERISTICS OF THE TRANSISTOR WITH (A) PTCDI-C8 AND (B) PENTACENE AS

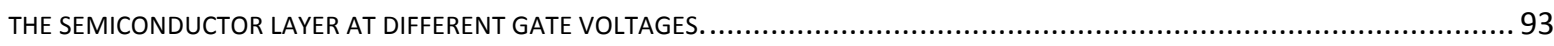

FIGURE 8.29 - CHANGE OF THE I IS UNDER ILLUMINATION VERSUS GATE VOLTAGE FOR THE PENTECENE-BASED TRANSISTORS. ................94

FIGURE 8.30 - MEASURED AND FITTED X-RAY DATA FOR LIF/PTCDI-C8 AND LIF/PENTACENE BILAYERS.....................................95

FIGURE 8.31 - OUTPUT CHARACTERISTICS OF THE ORGANIC THIN FILM TRANSISTORS BASED ON (A) LIF/PTCDI-C8 AND (B)

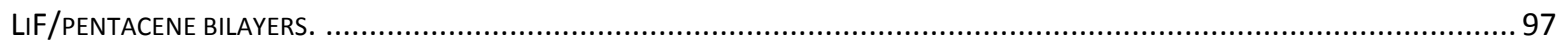

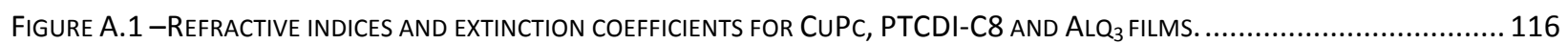

\section{LIST OF TABLES}

TABLE 1 - SUMMARY OF THE POWER CONVERSION EFFICIENCY, FILL FACTOR, OPEN CIRCUIT VOLTAGE AND SHORT CIRCUIT CURRENT FOR

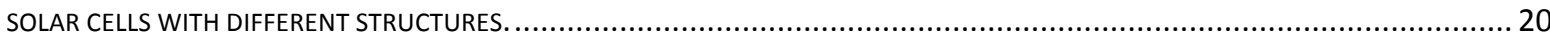

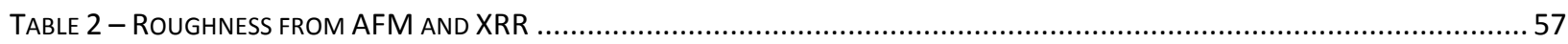

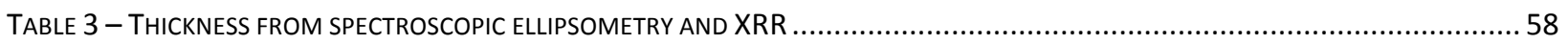


$\mathrm{Al}$

$\mathrm{Al}_{2} \mathrm{O}_{3}$

AFM

$\mathrm{a}-\mathrm{Si}: \mathrm{H}$

BHJ

C60

cat-CVD

CVD

CMOS

$\mathrm{CuPc}$

$\mathrm{eV}$

F-N

FTIR

HOMO

HWCVD

ITO

LCD

$\mathrm{LiF}$

LUMO

$\mathrm{MoO}_{3}$

$\mathrm{nm}$
Aluminum

Aluminum oxide

Atomic Force Microscopy

Amorphous Hydrogenated Silicon

Bulk Heterojunction

Fullerene

catalytic Chemical Vapor Deposition

Chemical Vapor Deposition

Complementary Metal Oxide Semiconductor

Copper Phthalocyanine

Electron Volt

Fowler-Nordheim

Fourier Transform Infrared Spectroscopy

Highest Occupied Molecular Orbital

Hot-Wire Chemical Vapor Deposition

Indium tin oxide

Liquid Crystal Display

Lithium Fluoride

Lowest Unoccupied Molecular Orbital

Molybdenum trioxide

Nanometer 


\begin{tabular}{|c|c|}
\hline NREL & National Renewable Energy Laboratory \\
\hline OLEDs & Organic Light Emitting Diodes \\
\hline OTFTs & Organic Thin Film Transistors \\
\hline OSCs & Organic Solar Cells \\
\hline OVPD & Organic Vapor Phase Deposition \\
\hline P3HT & Poly(3-hexylthiophene-2,5-diyl) \\
\hline PCBM & [6,6]-Phenyl C61 butyric acid methyl ester \\
\hline PECVD & Plasma Enhanced Chemical Vapor Deposition \\
\hline PEDOT:PSS & Poly(2,3-dihydrothieno-1,4-dioxin)-poly(styrenesulfonate) \\
\hline PHJ & Planar Heterojunction \\
\hline PTCDI-C8 & $\mathrm{N}, \mathrm{N}^{*}$-dioctyl-3,4,9,10-perylene tetracarboxylic diimide \\
\hline SEM & Scanning Electron Microscopy \\
\hline $\mathrm{Si}$ & Silicon \\
\hline $\mathrm{Si}_{3} \mathrm{~N}_{4}$ & Silicon Nitride \\
\hline $\mathrm{SiH}_{4}$ & Silane \\
\hline TEM & Transmission Electron Microscopy \\
\hline TFT & Thin Film Transistor \\
\hline UHV & Ultra-High Vacuum \\
\hline VTE & Vacuum Thermal Evaporation \\
\hline $\mathrm{V}_{2} \mathrm{O}_{5}$ & Vanadium Pentoxide \\
\hline XRD & X-ray Diffraction \\
\hline XRR & X-ray Reflectivity \\
\hline
\end{tabular}




\section{CHAPTER 1: INTRODUCTION}

Organic semiconductors as a new class of materials have attracted significant attention in the last few years for their use in semiconductor devices. Due to the many advantages including but not limited to low production cost, mechanical flexibility, large area devices and low-temperature fabrication, much effort has been devoted to the development of organic electronic devices such as organic light emitting diodes (OLEDs), organic solar cells (OSCs) and organic thin film transistors (OTFTs).

In order to manufacture organic electronic devices with high performance, more detailed studies of the structure and the morphology of the organic materials as well as the underlying physical charge transport mechanisms are warranted. Additionally, growth, deposition and assembly processes need to be established and optimized for the organic semiconductor technology.

Since structural properties of organic materials determine the optical and electrical characteristics of the device application, fundamental questions about these properties should be answered in order to optimize device performance. For instance, high-efficient OTFTs require materials with high charge carrier mobility. However, low charge carrier mobility of the organic semiconductors is one of the major problems that has hindered the development of organic transistors that are comparable in efficiency to traditional transistors [1]. Accordingly, much effort has been devoted to the synthesis and the development of new organic materials with higher carrier mobility. The parameters that determine the charge carrier mobility of the device include the structure of the first organic layer at the organic-dielectric interface as well as the morphology and the structural order of the other organic layers. It has been shown that, in thin 
film geometry with a single crystalline film, the optimum charge carrier transport can be achieved due to the presence of maximized intermolecular overlap of the electronic $\pi$-orbitals of adjacent molecules [2-4]. On the other hand, for other devices such as OLEDs and OSCs, the material morphology and structure at the organic-organic interface plays a crucial role in the device efficiency. For example in OSCs, in order to create and harvest as many excitons as possible, there needs to be a large interfacial area between the donor and the acceptor materials. To achieve this feat, different approaches such as co-deposition of the donor and the acceptor materials, layered structures and comb-shaped architecture of the active layers in the hybrid devices have been utilized. The aforementioned approaches signify the importance of the morphology and the structure of the organic materials as well as the device architecture in optimizing the performance of the device depending on its application. To summarize, charge carrier transport within the organic thin films as well as charge carrier injection between organic layers and organic-inorganic materials such as metal or dielectric layers are crucial factors in determining the efficiency of organic electronic devices. These parameters rely largely on the molecular structure, morphology and ordering of the organic thin films. Therefore, a profound understanding of the structure of organic materials as well as the properties of the interfacial layers is crucial to enhancing the performance of these devices.

In this work, several organic thin films have been prepared using the thermal evaporation method. Their morphology and structural properties have been studied by a combination of various techniques including atomic force microscopy, X-ray reflectivity, spectroscopic ellipsometry and transmittance measurements. Based on the produced organic thin films, organic semiconductor devices such as OTFTs, OLEDs and OSCs have been fabricated and their electrical and optical properties have been characterized. Moreover, the effect of morphology 
and structure of the organic thin films on the organic device performance has been studied. This work focuses on a material known as PTCDI-C8, which is a small molecule material regarded as an n-type semiconductor with a relatively high charge carrier mobility $[5,6]$. Molecular structure and packing of this material in thin films on technologically-relevant substrates such as silicon dioxide have been investigated to understand the origin of such high charge carrier mobility.

In this report, chapters 2-5 include literature review relevant to this work. In chapter 2, a summary of several types of organic semiconductor materials is presented. Chapter 3 describes the physics and the structure of organic solar cells and organic thin films transistors. Chapter 4 gives an overview of several different deposition techniques used to fabricate organic electronic devices. Chapter 5 describes the primary techniques used to characterize the organic materials and the fabricated organic devices. Chapter 6 describes the specific methods used in fabrication of the organic solar cells and organic thin film transistors studied in this work. Chapter 7 discusses the experimental results. Finally, Chapter 8 is a summary of the findings of this work. 


\section{CHAPTER 2: ORGANIC SEMICONDUCTOR MATERIALS}

Conjugated polymers and small molecules are the two classes of organic semiconductor materials that have been commonly used in the organic electronic devices. The following sections provide an overview of the two classes as well as a description of the specific materials used in this work.

\subsection{POLYMERS}

\subsubsection{Overview}

Polymers are large molecules with high molecular weight composed of a large number of small repeatable units called monomers. There are both naturally occurring and synthetic polymers. Proteins, cellulose and latex are among naturally occurring polymers. Synthetic polymers are produced commercially and have a wide range of properties and applications.

Polymers are constructed by chemical reactions that allow sequential joining of a large number of monomers by covalent chemical bonds to form a chain. When a polymer is derived from more than one monomer, it is referred to as a copolymer or heteropolymer. Based on the nature and the structure of the chemical bonds between its monomers, polymers can be classified into two groups: conjugated and non-conjugated. Both conjugated and non-conjugated polymers have similar mechanical properties but differing electrical behaviors. Polymers were originally considered to be promising insulators and were used as photoresist materials in the semiconductor industry. However, with the 1977 discovery that doping of polyacetylene enables relatively high conductivity, a new era began in the exploration of organic electronics and optoelectronics [7]. 


\subsubsection{Conjugated and non-conjugated polymers}

Polymers are composed of repeating structural units called monomers. Carbon and hydrogen atoms are the basic elements of the majority of monomers. Electrical properties of different type of polymers are defined based on their chemical bonding. For non-conjugated polymers, the bond between the central carbon and each of its attached hydrogen atoms is formed by a covalent interaction between sp3 hybrids with the carbon atoms and 1s orbital with the hydrogen atom. In this bonding structure, it requires a large energy to move an electron from one bonding orbital to the equivalent anti-bonding orbital. In other words, there is a large energy gap between occupied and unoccupied bands of non-conjugated polymers, which results in the insulating properties of these materials.

On the other hand, the chemical bond structure of conjugated polymers is based on a backbone structure consisting of alternating single and double carbon bonds. This kind of bonding results in a " $\pi$-conjugated network" that leads to a relatively small energy gap. In conjugated polymers, some parts of the chemical bonding are formed by sp2 hybrids with carbon atoms and $1 \mathrm{~s}$ function with the hydrogen atoms. The other parts of chemical bonding are formed by $\mathrm{p}$ function with the carbon atoms, which constitutes the $\pi$ bonds between the carbon atoms. Since less energy is required for moving an electron from a $\pi$ bond to an anti-bonding one, the energy gap between occupied and unoccupied orbitals is relatively smaller than that of non-conjugated polymer [8]. In a conjugated polymer, the alternating single and double carbon bonds lead to electron delocalization. The delocalized electrons form a band structure, which exhibits semiconducting or metallic properties. These electrons will also act as charge carriers and move along the polymer chain, allowing the creations of a conductive polymer. 


\subsection{SMALL MOLECULES}

Semiconducting small molecules are low molecular weight organic compounds that include aromatic hydrocarbons. They are generally sublimed in a vacuum system whereas conjugated polymers are dissolved in appropriate solvents and applied by solution processing methods, such as spin-coating or ink-jet printing. Since small molecules can be evaporated, highly complex multi-layer structures can be constructed compared to the structures based on polymers. The band gap in organic semiconductors is determined by the difference between the highest occupied molecular orbital (HOMO) and the lowest unoccupied molecular orbital (LUMO). Electrical and optical properties of organic materials can easily be tuned to a certain extent by controlling their band gap via synthesizing different molecules with different size, atomic arrangement and functional groups. Pentacene, tetracene and rubrene are among common organic small molecules that include a polycyclic aromatic structure. The application of solution processing method along with vacuum sublimation technique can result in amorphous or polycrystalline films with variable degree of disorder. Pentacene, CuPc and PTCDI, all with relatively high carrier mobility, are the most common organic materials that have been used as the active layer in organic thin film transistors and solar cells. A summary of the structure and properties of these materials will be presented in the next sections.

\subsection{MATERIALS STRUCTURE}

In order to improve the thin film transport properties, much work has been done to investigate and understand the effect of the deposition conditions on the structural, electrical and mechanical properties of organic thin films. Crystal structure, molecular arrangement and the 
surface morphology of the organic layer are some of the main factors that should be considered when trying to fabricate organic electronic devices with high performance.

\subsubsection{Pentacene}

Pentacene, as a crystalline organic molecular material, has attracted significant attention recently due to its relatively high carrier mobility of single crystal. This makes pentacene a suitable candidate for use in the active layer in organic flexible electronic devices $[9,10]$. Thin film pentacene is commonly produced by solution processing or thermal evaporation onto various substrates such as glass, mica or silicon. The density and the distribution of the charge carrier trapping sites (e.g. grain boundaries and dislocations) are some of the parameters that determine the film transport properties [11]. The chemical structure of pentacene has been presented schematically in Figure 2.1. Pentacene is a small molecule organic compound, composed of five benzene rings with a chain-like aromatic molecule with the molecular formula $\mathrm{C}_{22} \mathrm{H}_{14}$ and a molar weight of $278.36 \mathrm{~g} / \mathrm{mol}$. Pentacene single crystal has a triclinic structure with two molecules in the unit cell with the following lattice parameters: $a=0.628 \mathrm{~nm}, \mathrm{~b}=0.771 \mathrm{~nm}$, $\mathrm{c}=1.444 \mathrm{~nm}, \alpha=76.75^{\circ}, \beta=88.01^{\circ}, \gamma=84.52^{\circ}$ [12]. In thin-film forms, pentacene molecules pack into a layered structure forming a herringbone pattern within the layer [13-15]. Solid phase transformation from the orthorhombic phase to the thin-film phase and then to the triclinic bulk phase is believed to take place in pentacene thin films due to the thickness increment. More grain boundaries exist in the thin film pentacene at low thickness because of the high nucleation density that leads to small grain size. As the thickness of the film increases, the grain boundaries enlarge and the pentacene molecules will grow on the inclined grain boundaries. This is the stage where the growth phase changes from the orthorhombic phase to the thin-film phase. At the 
higher thickness, the thin-film phase will change to the triclinic bulk phase with less but larger grain boundaries [16].
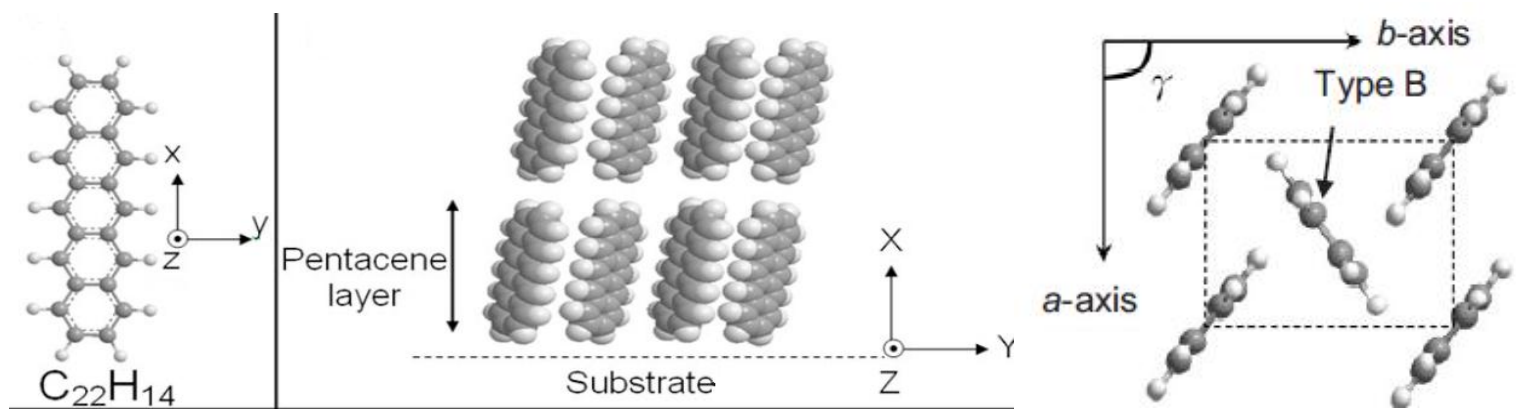

Figure 2.1 - Chemical representation of pentacene molecule (left), pentacene thin film with layered structure (middle) [15], schematic of a unit cell and herringbone packing of pentacene molecules (right) [12].

\subsection{2. $\quad \mathrm{CuPc}$}

Phthalocyanines are porphyrin derivatives with planar molecules consisting of four isoindole subunits linked together through nitrogen atoms $\left(\mathrm{C}_{8} \mathrm{H}_{7} \mathrm{~N}\right)$ as presented in Figure 2.2. Copper Phthalocyanine $(\mathrm{CuPc})$ is an organic molecule with the molecular formula $\mathrm{C}_{32} \mathrm{H}_{16} \mathrm{CuN}_{8}$ and a molecular weight of $576.1 \mathrm{~g} / \mathrm{mol}$. This molecule is thermally stable and therefore can be sublimated in vacuum. It has shown p-type semiconductor behavior and has been considered as a potential candidate for organic devices [17-20]. Several studies have been performed trying to investigate the bulk structure of $\mathrm{CuPc}$ [21]. For the thin-film structure of $\mathrm{CuPc}$, two major polymorphs ( $\alpha$ - and $\beta$ - form) exist. The $\alpha$-form is the metastable form of the crystal structure with the lattice parameters of $\mathrm{a}=2.59 \mathrm{~nm}, \mathrm{~b}=0.34 \mathrm{~nm}, \mathrm{c}=2.39 \mathrm{~nm}, \alpha=90^{\circ}, \beta=90.4^{\circ}$ and $\gamma=$ $90^{\circ}$ [21]. The $\beta$-form, which is the stable phase, can only be achieved at high temperatures ( $~$ 
$240{ }^{\circ} \mathrm{C}$ ) and beyond a critical thickness of about $800 \mathrm{~nm}$ [22]. It has the following lattice parameters: $\mathrm{a}=1.46 \mathrm{~nm}, \mathrm{~b}=0.48 \mathrm{~nm}, \mathrm{c}=1.95 \mathrm{~nm}, \alpha=90^{\circ}, \beta=121^{\circ}$ and $\gamma=90^{\circ}$ [23].

a)<smiles>c1c[nH]c2cccc-2c1</smiles>

b)

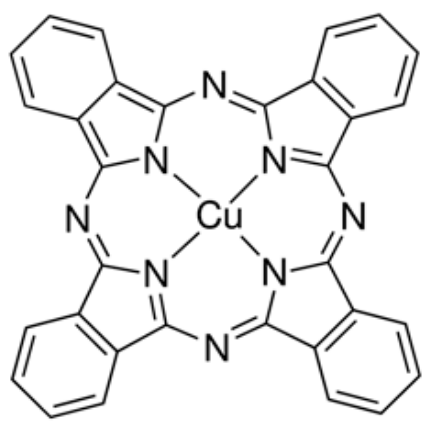

c)
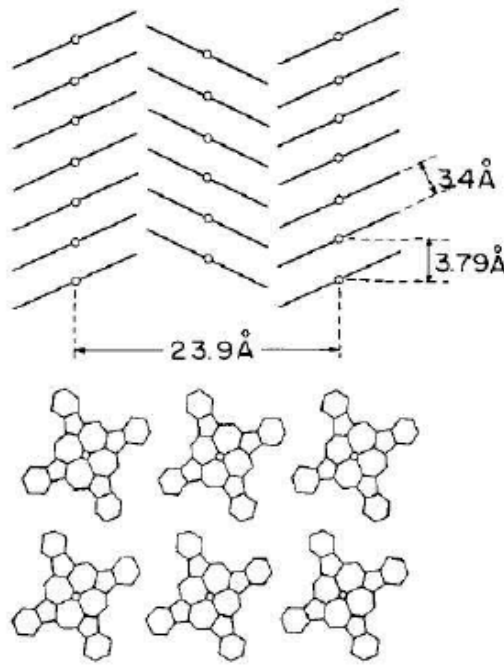<smiles>[Li][Ca]</smiles>

d)<smiles>[AsH]</smiles>
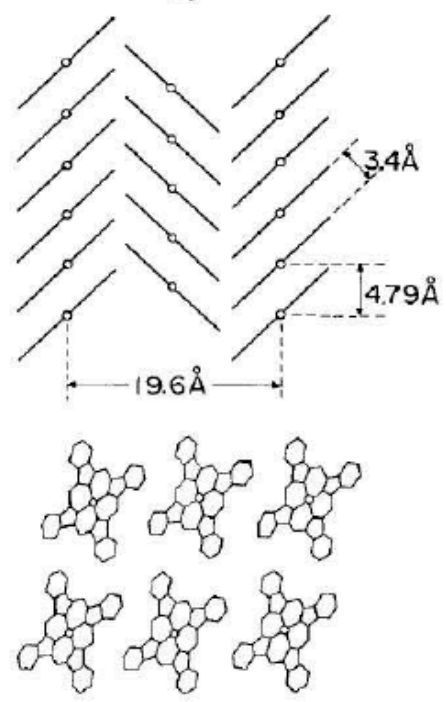

Figure 2.2 - (a) Chemical representation of the isoindole subunits, (b) Copper Phthalocyanine $(\mathrm{CuPc})$ molecule, molecular arrangement in $\mathrm{CuPC}$ thin films of the, (c) $\alpha$-form, and (d) $\beta$ - form [24].

\subsubsection{PTCDI-C8}

Perylene Tetracarboxylic Diimide derivatives are among the most promising small organic molecules to fabricate organic thin film transistors (OTFTs) and organic solar cells (OSCs) $[25,26]$. Their optical and electrical properties can be modified by attaching different functional groups at specific molecular positions [27, 28]. PTCDI-C8 (N,N*-dioctyl-3,4,9,10perylene tetracarboxylic diimide) is an organic molecule with molecular formula $\mathrm{C}_{40} \mathrm{H}_{42} \mathrm{~N}_{2} \mathrm{O}_{4}$ and molecular weight of $614.77 \mathrm{~g} / \mathrm{mol}$ which belongs to the perylenes family. This small 
molecule has attracted much attention due to its high electron mobility ranging from $0.6 \mathrm{~cm}^{2} / \mathrm{Vs}$ to $1.7 \mathrm{~cm}^{2} / \mathrm{Vs}[29,30]$. Figure 2.3 shows the chemical structure of the PTCDI-C8 molecule. Various works have attempted to investigate and understand the structure and properties of the thin film PTCDI under different growth conditions. [20-23]. In many of these attempts, in order to study the three-dimensional thin-film structure of the PTCDI-C8, a layer with a thickness about $180 \AA$ of this organic substance has been evaporated by organic vapor phase deposition (OVPD) in ultrahigh vacuum (UHV) on $\mathrm{Al}_{2} \mathrm{O}_{3}(11-20)$ substrate and at $150{ }^{\circ} \mathrm{C}$. The evaporated thin film has been characterized using threedimensional X-ray crystallography. X-ray results show that PTCDI-C8 forms a smoothlayered film with an extraordinary crystalline order on the $\mathrm{Al}_{2} \mathrm{O}_{3}$ substrate. Lattice properties of the thin film structure are found to be significantly different from the bulk structure. PTCDI-C8 thin films have triclinic unit cell with the plane of the aromatic core

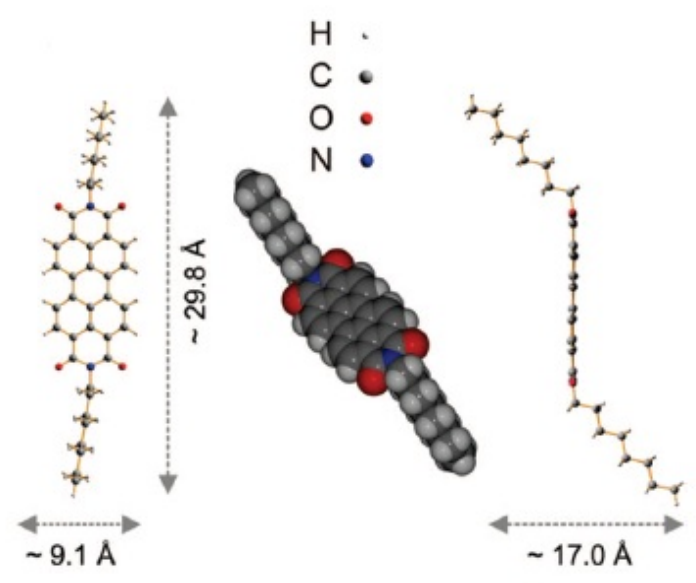

Figure 2.3 - Chemical representation of PTCDI-C8 molecule [31]. tilted by $67^{\circ}$ with respect to the surface plane. The primitive triclinic unit cell of PTCDI has the following lattice parameters: $\mathrm{a}=0.9 \mathrm{~nm}, \mathrm{~b}=0.489 \mathrm{~nm}, \mathrm{c}=2.165 \mathrm{~nm}, \alpha=95.0^{\circ}, \beta=100.7^{\circ}$ and $\gamma=112.8^{\circ}[31]$ 


\subsection{ORGANIC SOLAR CELLS}

\subsubsection{Overview}

Solar cells are unique non-polluting sources of renewable energy. They require minimal maintenance and can be scaled from microelectronic power sources to utility-scale power generators. Significant progress in fabrication and optimization of organic solar cells (OSCs) has been made during the last decade [32]. The underlying reasons for the popularity of OSCs are multifold: compatibility with flexible substrates; low processing temperature; large area devices; light weight; and low-cost fabrication processes such as evaporation, spin-coating and printing [33].

In addition to all of the abovementioned benefits, organic materials have the ability to minimize some of the major loss processes, particularly thermalization, that occur in inorganic solar cells. In a typical solar cell, there are energy losses due to non-absorption of below band gap photons, thermalization, junction voltage

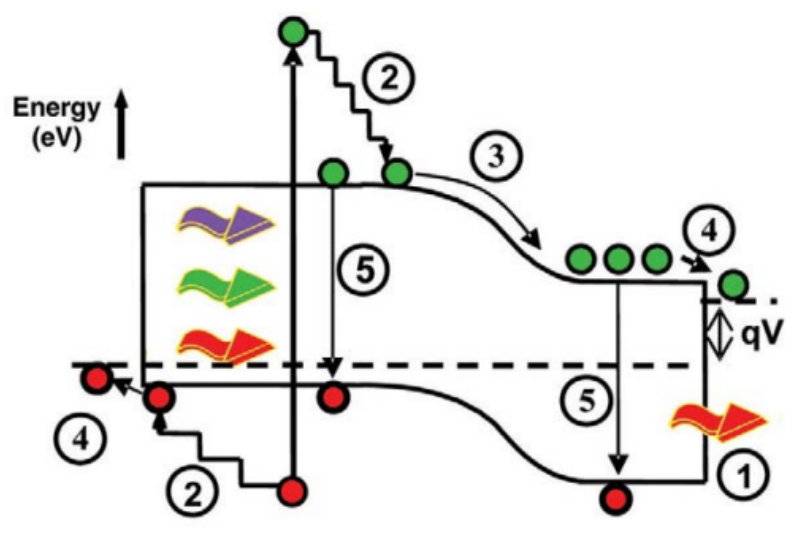

Figure 3.1 - A summary of the different loss processes in a typical solar cell [34].

loss, contact voltage loss and charge recombination loss [34]. A summary of these loss processes is presented in Figure 3.1. Among them, thermalization loss can be minimized by using larger band gap materials. To illustrate this point, Figure 3.2 presents the solar energy spectrum [35]. As can be seen in this figure, for solar 
cells based on silicon with the band gap of $1.1 \mathrm{eV}$, most of the absorption will take place at approximately $1.1 \mu \mathrm{m}$ where the intensity of the light is relatively low. Therefore, the bulk of the solar energy will be lost to thermalization. In order to minimize thermalization loss, III-Nitride semiconductor alloys have been studied. By tuning the band gap of these alloys, which can be a difficult and expensive process, different ranges of solar energy can be absorbed. However, for organic materials, it is relatively much easier and more inexpensive to tune the band gap by synthesizing different organic materials. This is a major benefit of using organic materials instead of inorganic materials in fabrication of solar cells.

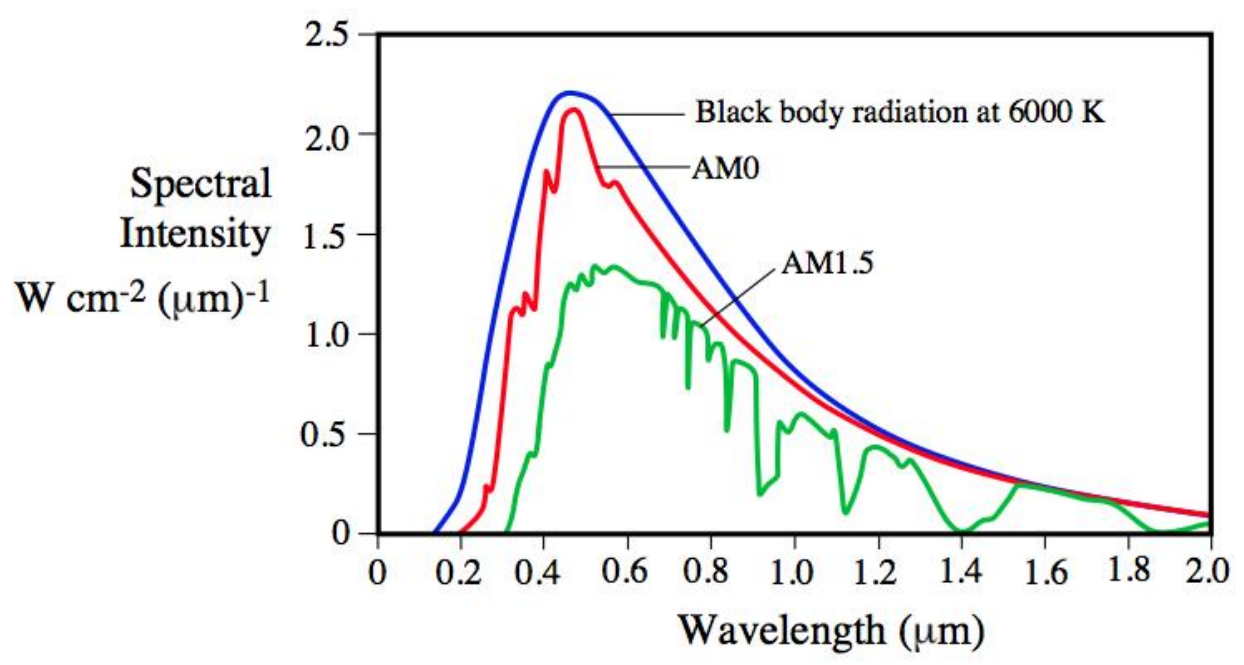

Figure 3.2 - Solar energy spectrum [35].

Another major advantage of organic materials for solar cells is their potential ability to be utilized in thin film devices in a cost-efficient manner. For silicon solar cells, due to their low absorption coefficients, thin film devices will not provide high efficiency of light absorption. However, organic materials have relatively large absorption coefficients in excess of $10^{5} \mathrm{~cm}^{-1}$, allowing them to be effective at light absorption in a thin film structure. Overall, the ability of organic materials to be used in thin film solar cells can reduce the production cost of future solar 
panels compared to the current silicon solar panels, in which the absorber material is responsible for approximately $50 \%$ of the production cost [36]. Additionally, since silicon is a rare earth material with fluctuating pricing depending on the geopolitical climate of the exporting countries, having the ability to synthesize organic materials used in solar cells would provide stability of the production costs in this booming industry.

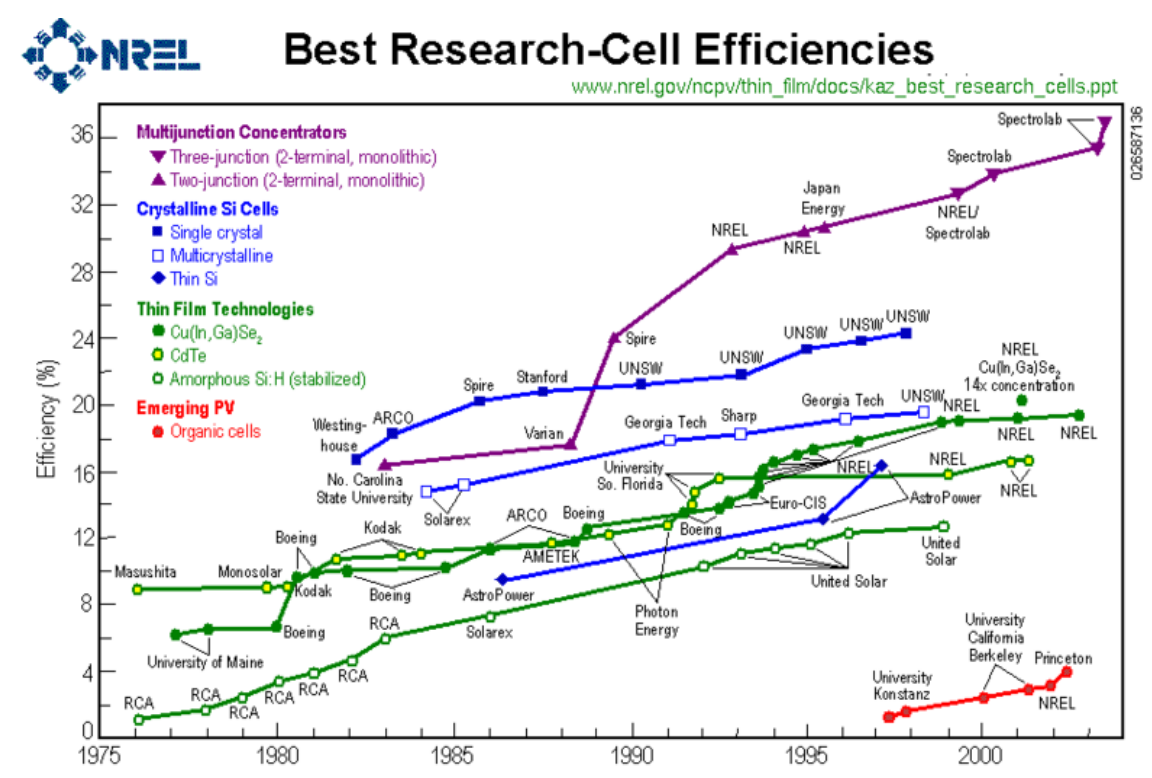

Figure 3.3 - An overview of the power conversion efficiency of organic solar cells provided by the National Renewable Energy Laboratory (NREL) [38].

Even though significant progress has been made in fabricating high performance OSCs for the past 20 years [37], the power conversion efficiency of OSCs continues to be lower compared to inorganic solar cells. An overview provided by the National Renewable Energy Laboratory (NREL) is presented in Figure 3.3 in order to compare the power conversion efficiencies of OSCs with inorganic solar cells [38]. Various approaches including optimizing the morphology of the active layers [39, 40], introducing new materials as the donor and 
acceptor $[41,42]$ and new device structures such as tandem structure $[43,44]$ have been adopted to improve the efficiency of the OSCs. Devices based on conjugated polymers have been shown to provide power conversion efficiencies up to $6 \%$ and $6.5 \%$ for single-layer and tandem solar

cells, respectively [45]. Using a bulk heterojunction structure with an active area of $1.13 \mathrm{~cm}^{2}$, a record efficiency of approximately $8.5 \%$ has been reported recently (Jan. 2012) by Solarmer Energy, Inc. [46]. The highest efficiency for OSCs that has been reported recently (Feb. 2012) by Konarka Technologies, Inc. is approximately 9\% [47]. More detail about the basic principles of OSCs and the different device structures will be presented in the following sections.

\subsubsection{Basic principles}

A fundamental difference between organic and conventional inorganic solar cells is that in organic cells, the light absorption results in the formation of excitons rather than free electrons and holes. An exciton in an organic semiconductor can be considered a tightly bound electron hole pair due to Coulombic force in the molecules. For a general donor/acceptor solar cell, photoinduced charge generation and collection processes can be explained in the following steps, as shown in Figure 3.4. The absorbed photons from the incident light generate bound electron hole pairs (exciton) in the donor (p-type) material. If the diffusion length of the generated exciton is long enough, it will diffuse into the donor/acceptor interface. Due to the difference between the work function of the electrodes, an internal electric field will be established. Excitons at the donor/acceptor interface will separate due to the internal field. Once the excitons are separated, free excess charge carriers will be collected at the respective electrodes. Finally, the charge carriers will be extracted to an external circuit where it can be used as electrical energy [48]. 


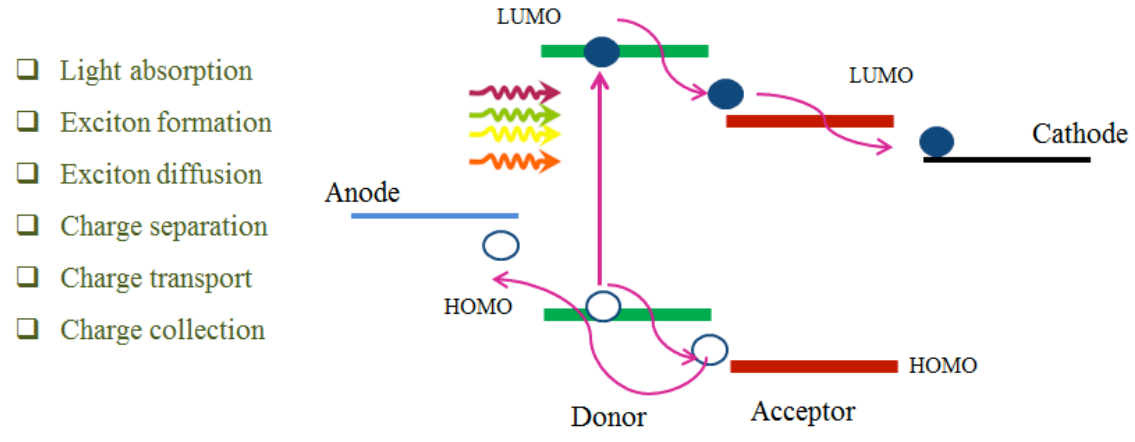

Figure 3.4 - Photoinduced charge generation and collection processes for a typical donor/acceptor bilayer solar cell.

A conventional structure of a solar cell is a $p-n$ junction sandwiched between two metals as the electrode contacts with different work functions. One of the electrodes has to be a transparent electrode to allow light absorption. An ideal solar cell can be considered as an illuminated diode where free excess charge carriers are generated by light. The drift current components of the diode increase due to the photo-generated charge carriers. This photogenerated current $\left(\mathrm{I}_{\mathrm{Ph}}\right)$ is limited by generation and recombination of

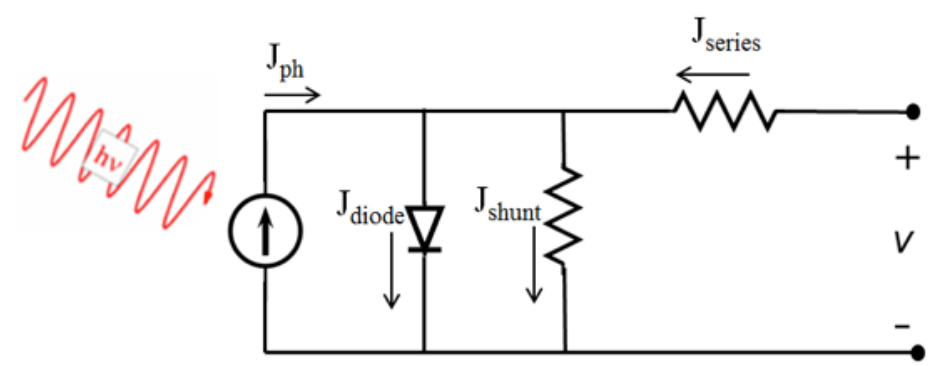
the charge carriers due to light Figure 3.5-Equivalent circuit for a solar cell with shunt absorption. However, for a and series resistors.

realistic model of a solar cell, the resistances appearing in the operation mode should be added to the equivalent circuit and the Shockley diode equation has to be adjusted accordingly. The replacement circuit of a real-life solar cell has been shown in Figure 3.5. The series resistance 
$\left(\mathrm{R}_{\mathrm{s}}\right)$ is attributed to the losses in the bulk materials or the contacts and their interfaces. The shunt resistance $\left(\mathrm{R}_{\mathrm{p}}\right)$ is typically due to the charge conduction through the defects and shorts in the material. Low shunt resistance causes power loss in the solar cells by providing an alternate current path for the photogenerated current.

Ideally, current in a solar cell can be modeled as Equation 3.1,

$$
I=I_{s a t}\left(\exp \left(\frac{q V}{k T}-1\right)\right)-I_{p h}
$$

where $\mathrm{I}_{\text {sat }}$ is the saturation current under reverse bias and $\mathrm{I}_{\mathrm{ph}}$ is the photogenerated current.

For real-life devices, current can be determined using the following equation which considers both serial and shunt resistance losses,

$$
I=I_{s a t}\left(\exp \left(\frac{q V}{n k T}-1\right)\right)+\frac{V-I R_{S}}{R_{P}}-I_{p h}
$$

where $\mathrm{n}$ is the diode ideality factor, which is equal to one for ideal diodes.

The relationship between the open circuit voltage $\left(\mathrm{V}_{\mathrm{OC}}\right)$ and the short circuit current $\left(\mathrm{I}_{\mathrm{SC}}\right)$ can be determined when $R_{S}=0$ and $R_{P}=\infty$, with $I=0$ for $V_{O C}$ and $I_{p h}=I_{S C}$ :

$$
V_{O C}=\frac{n k T}{q} \ln \left(\frac{I_{S C}}{I_{\text {sat }}}+1\right)
$$

As can be seen from Equation 3.3, a small shunt resistance reduces $V_{O C}$, and $I_{S C}$ is reduced by the series resistance $\mathrm{R}_{\mathrm{S}}$.

The plot of the power $(\mathrm{P}=\mathrm{IV})$ versus applied voltage $(\mathrm{V})$ can be used to calculate the maximum power that can be delivered to the external resistance by a solar cell. The maximum value for the power will occur between $\mathrm{I}_{\mathrm{SC}}$ and $\mathrm{V}_{\mathrm{OC}}$ and will be zero at these two points. The voltage and the current at this maximum power point $\left(\mathrm{V}_{\max }\right.$ and $\left.\mathrm{I}_{\max }\right)$ are presented in Figure 3.6. 
Fill factor (FF) and power

conversion efficiency $(\eta)$ are the two key parameters in evaluating the performance of solar cells. The FF is the ratio of the generated maximum power to the product of the $\mathrm{V}_{\mathrm{OC}}$ and $\mathrm{I}_{\mathrm{SC}}$, as calculated in Equation 3.4.

$$
F F=\left|\frac{V_{\text {max }} I_{\text {max }}}{V_{O C} I_{S C}}\right|
$$

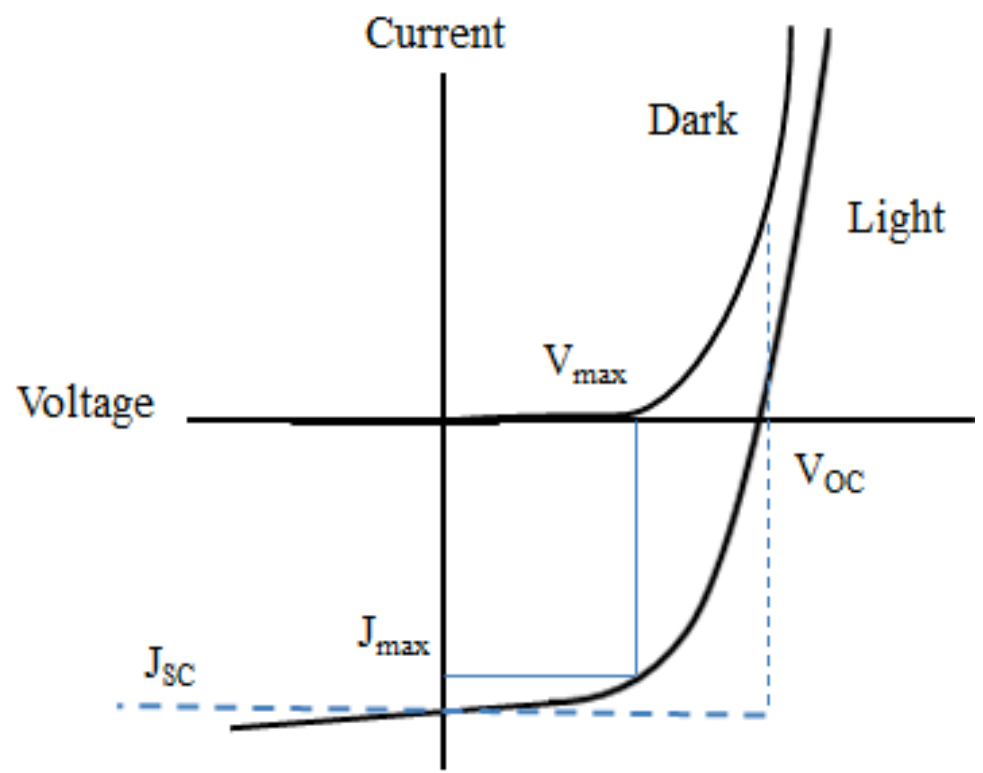

Figure 3.6 - Typical I-V plot of a solar sell under dark and light conditions.

A typical I-V plot of a solar sell under dark and light conditions is presented in Figure 3.7. The generated maximum power $\left(\mathrm{P}_{\max }\right)$ results from the absolute value of the product of maximum current and voltage at the maximum power point $[49,50]$. With the introduction of the fill factor, the power conversion efficiency $(\eta)$ of a solar cell can be calculated as the ratio between the maximum generated power of the cell and the power density of the incident light $\left(\mathrm{P}_{\text {in }}\right)$, as shown in Equation 3.5.

$$
\eta=\frac{P_{\max }}{P_{i n}}=F F \frac{V_{O C} I_{S C}}{P_{i n}}
$$

Other parameters that can be extracted from the I-V curves of a solar cell are the series and the shunt resistances. The slope of the I-V curve in the vicinity of the open circuit voltage indicates the value for shunt resistance since the effect of the series resistance is negligible near 
this point $\left(\mathrm{V}_{\mathrm{OC}}\right)$. Similarly, since the effect of the shunt resistance is negligible near the short circuit current, series resistance can be calculated from the slope of the I-V curve in the vicinity of $\mathrm{J}_{\mathrm{SC}}$. The diode ideality factor (n) and the saturation current at reverse bias $\left(\mathrm{I}_{\text {sat }}\right)$ are the other two parameters that can be extracted from the I-V curve of a solar cell. Under dark conditions, the I-V plot of a solar cell can be modeled as shown in Equation 3.6.

$$
I=I_{\text {sat }}\left(\exp \left(\frac{q V}{n k T}-1\right)\right)
$$

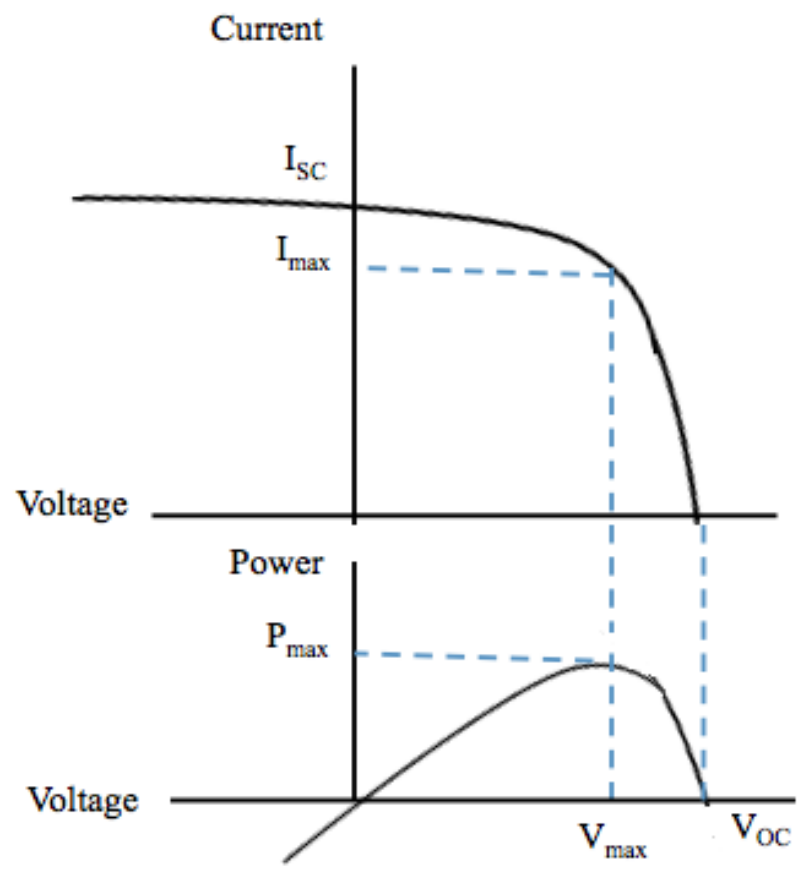

For applied voltages larger than 50 to 100 $\mathrm{mV}$, the term -1 in the above equation Figure 3.7 - Maximum power for an I-V sweep of a solar cell.

can be ignored, resulting in Equation 3.7.

$$
I=I_{s a t} \exp \left(\frac{q V}{n k T}\right)
$$

Taking the natural log of each side of the above equation gives Equation 3.8.

$$
\operatorname{Ln}(I)=\operatorname{Ln}\left(I_{s a t}\right)+\left(\frac{q}{n k T}\right) V
$$

By plotting the above equation, $\operatorname{Ln}\left(\mathrm{I}_{\mathrm{sat}}\right)$ can be calculated from the intercept of the plot of the natural $\log$ of the current versus voltage, and the ideality factor (n) can be calculated from the slope. 


\subsubsection{Solar cell architectures}

Complete light absorption is nearly possible for sufficiently thick organic layers due to the typically high absorption coefficients of organic materials $\left(\alpha \approx 10^{5} \mathrm{~cm}^{-1}\right)$ [51]. Additionally, it has been shown that charge transfer at the donor/acceptor interface occurs in a timescale of a few hundred femtoseconds, which allows high charge transfer efficiency [52]. If the charge carrier mobility is sufficiently high, high charge collection efficiency can be achieved in planar heterojunction solar cells [53]. However, short exciton diffusion length of organic materials (few nanometers) is one of the limiting parameters that hinders the ability to enhance the efficiency of solar cells $[51,54]$. Different solar cell architectures have been proposed and fabricated to overcome this problem [51,53,55-57]. Using these concepts, power conversion efficiencies exceeding 5\% can be achieved for organic solar cells [58-60]. Different possible device architectures are presented in Figure 3.8. The planar heterojunction (PHJ) with a sharp interface between the donor and the acceptor is the simplest structure, as shown in Figure 3.8 (a). The effective interfacial area between the donor and the acceptor can be increased by creating a roughened surface or a comb-shape structure.

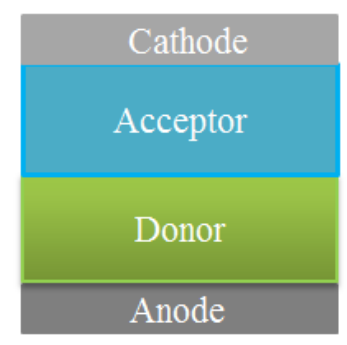

a)

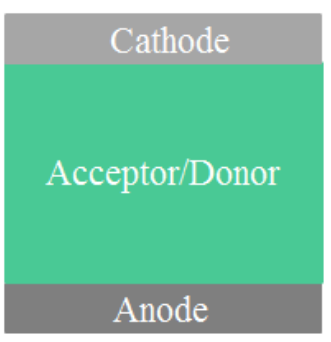

b)

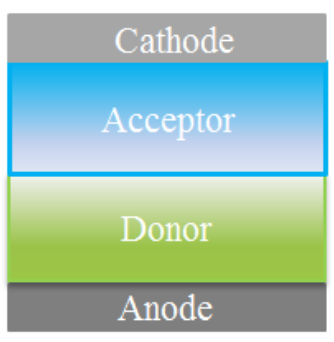

c)

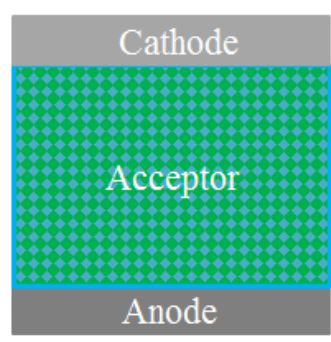

d)

Figure 3.8 - Examples of different possible solar cell architectures. 
In a bulk heterojunction (BHJ) structure with a mixture of the donor and the acceptor materials, the interface between the two organic materials is distributed over the entire blended film (Figure 3.8 (b)). However, there is a very low control over this distribution. Different approaches such as a compositional gradient or a phase-separated system (Figures 3.8 (c) and 3.8 (d)) have been introduced to control the distribution of the organic materials in the BHJ structure. Compositional gradient structures can be achieved using techniques such as organic vapor phase deposition, which provides a higher degree of deposition control. Phase separation morphology can be controlled by post-deposition annealing in the polymer and the molecular solar cells [52, 61]. Such a distributed interface allows for overcoming the exciton short diffusion length problem. However, this may affect the transport efficiency of both types of the charge carriers to the electrodes. A summary of the performance of solar cells with different architectures is presented in Table 1.

Table 1 - Summary of the power conversion efficiency, fill factor, open circuit voltage and short circuit current for solar cells with different structures.

\begin{tabular}{|c|c|c|c|c|c|}
\hline \multicolumn{2}{|c|}{ Solar cell structure } & \multirow{2}{*}{$\frac{\mathrm{J}_{\mathrm{SC}}\left(\mathrm{mA} / \mathrm{cm}^{2}\right)}{1.2-4}$} & \multirow{2}{*}{$\frac{\mathrm{V}_{\mathrm{OC}}(\mathrm{V})}{0.5-0.53}$} & \multirow{2}{*}{$\frac{F F(\%)}{26-32}$} & \multirow{2}{*}{$\frac{\eta(\%)}{0.2-0.7}$} \\
\hline $\mathrm{CuPc} / \mathrm{C} 60$ & PHJ - without blocking layer & & & & \\
\hline CuPc:C60 & BHJ - without blocking layer & $4.1-6.8$ & $0.35-0.46$ & $31-37$ & $0.3-0.9$ \\
\hline $\mathrm{CuPc} / \mathrm{C} 60$ & PHJ - with blocking layer & $5.5-7.7$ & $0.5-0.56$ & $34-55$ & $1.3-2.3$ \\
\hline CuPc:C60 & BHJ - with blocking layer & 7.4-10.6 & $0.48-0.55$ & $22-33$ & $0.9-1.8$ \\
\hline
\end{tabular}




\subsection{ORGANIC THIN FILM TRANSISTORS}

\subsubsection{Overview}

Organic thin film transistors (OTFTs), due to their simple and low-cost fabrication processes and structural flexibility, have various applications such as in the switching elements of flat-panel displays and smart cards. However, the low carrier mobility in organic semiconductor materials compared to inorganic semiconductor materials and the difficulty of integrating organic devices into inorganic processing procedures have hindered the development of organic transistors that are comparable to traditional transistors $[4,62]$.

\subsubsection{Thin film transistors}

A thin film transistor (TFT) is one of the configurations of field-effect transistor (FET). Its operation is similar to the operation of a conventional FET, except that in TFT, the conducting channel is induced in the accumulation regime rather than through the formation of an inversion layer. A thin film transistor comprises a semiconductor layer as the active layer, a dielectric layer as the gate insulator, metallic contacts and a gate electrode. The semiconductor layer is typically a polysilicon or an amorphous silicon layer. These transistors are used as switching devices in flat-panel displays such as liquid crystal displays (LCDs). The four basic structures of the planar TFTs are shown in Figure 3.9. The deposition order of the layers determines the structure of the transistor. They can be fabricated in top or bottom gate contacts configuration with either top or bottom source-drain electrodes. If an n-type semiconductor is used as the active layer of the TFT, by applying a positive gate voltage, electrons will be induced into the channel and will create the accumulation layer. On the other hand, for a p-type 
semiconductor, holes will accumulate in the channel by applying a negative voltage of the gate. This is the region in which TFTs operate and the conducting channel will be generated.

\subsubsection{TFT materials and fabrication technologies}

Because of the high processing temperature and the limited size of the wafers, the conventional silicon technology based on crystalline silicon is incompatible with large area electronics. New techniques were developed to grow silicon over large surfaces at low temperature. Amorphous hydrogenated silicon $(\mathrm{a}-\mathrm{Si}: \mathrm{H})$ could be obtained by deposition of silicon from silane $\left(\mathrm{SiH}_{4}\right)$ gas using chemical vapor deposition (CVD) techniques that can be used for fabrication of transistors over large surfaces. Plasma enhanced chemical vapor deposition (PECVD) is the most commonly used method to deposit amorphous silicon over large
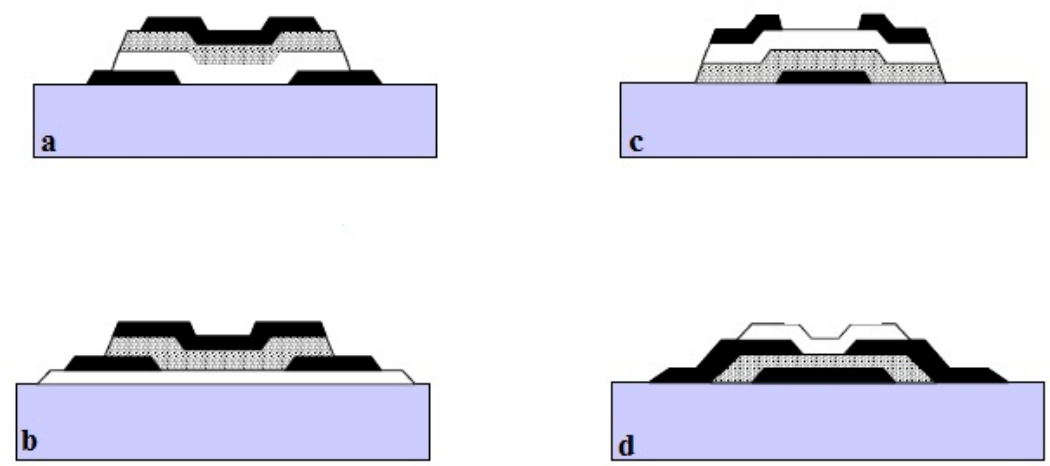

substrate

contacts insulator semiconductor

Figure 3.9 - Basic thin film transistors configuration, (a) top-gated top contacts configuration, (b) top-gated bottom contacts configuration, (c) bottom-gated top contacts configuration, (d) bottom-gated bottom contacts configuration [66]. 
areas at low temperatures of $450{ }^{\circ} \mathrm{C}$. Low temperature deposition allows the use of inexpensive substrates. The obtained material using this deposition method is amorphous silicon with incorporated hydrogen atoms. Amorphous silicon is a material with a disordered atomic structure that contains many dangling bonds. These dangling bonds may cause creation of defect states in the band gap of the amorphous silicon. Hydrogen atoms fill up some of these dangling bonds and decrease the density of the defect states, thereby allowing a-Si:H to have much better properties compared to other amorphous materials. In order to improve the electrical properties of this material, different techniques such as hot-wire chemical vapor deposition (HWCVD), also known as catalytic chemical vapor deposition (cat-CVD), have been used. In this technique, silane molecules are cracked into silicon, hydrogen and radicals such as $\mathrm{SiH}, \mathrm{SiH}_{2}$ and $\mathrm{SiH}_{3}$ by using a filament heated to about $1800{ }^{\circ} \mathrm{C}$. A polycrystalline silicon-hydrogenated film grows on the substrate surface from the gas phase with much better electrical properties compared to the amorphous hydrogenated silicon. Based on the utilized techniques, a different type of silicon, amorphous or polysilicon, will be deposited on a large area substrate and will be employed in producing TFTs $[63,64]$.

\subsubsection{Organic Thin Film Transistors (OTFTs)}

OTFT is a thin film transistor consisting of an organic layer as the semiconductor layer, a gate insulator layer and three electrical terminals. Silicon, glass and plastic are the commonly used OTFT substrates [65]. The resistance of the organic semiconductor layer, between the source and the drain contacts, is modulated by the applied voltage to the gate electrode. A simple way to understand the OTFT operation concept is to consider it as operating in a digital mode, similar to a switch. Under an applied gate bias, an electric field will be induced inside the gate- 
insulating layer. Due to the accumulation of the majority of the charge carriers at the insulatororganic interface, a significant increase in the conductivity of the channel will occur, resulting in the ON state of operation of the switch. In contrast, by inverting the polarity of the applied gate bias, due to a generated depletion region in the insulator-organic interface, the conductivity of the channel will decrease and the switch will be in the OFF state [66].

OTFTs have a structure similar to the conventional transistors with $\mathrm{SiO}_{2}$ as the gate insulating layer, but utilize an organic material as the conduction channel. In the devices based on this structure, heavily doped p-type or n-type silicon wafers are used as the gate electrodes and a thick layer of $\mathrm{SiO}_{2}$ at approximately $300 \mathrm{~nm}$ is deposited as the

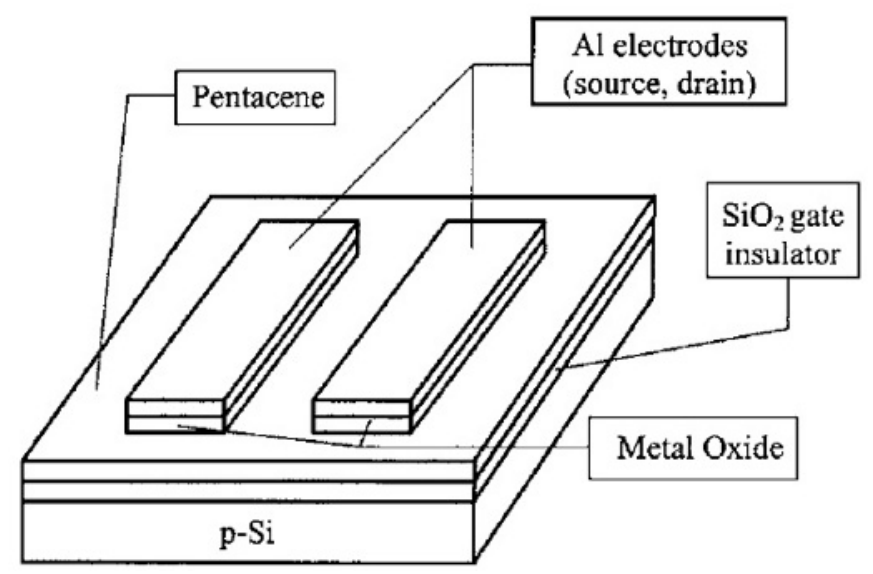

Figure 3.10 - Typical OTFT structure with $\mathrm{SiO} 2$ as the gate insulating layer [67].

gate insulating layer on top of the gate electrode. Active layer, which is the organic semiconductor layer, will be evaporated on top of the gate insulating layer and the drain and the source contacts will be deposited on top of the organic semiconductor. Figure 3.10 shows an OTFT structure with Pentacene used as the organic layer and Al as the drain and source electrodes [67]. In this work, $\mathrm{LiF}$ has been used as the gate insulating layer instead of the commonly used $\mathrm{SiO}_{2}$. This is the main difference between this work and the previous reports. Details about the structure of the device are presented in Chapter 6 . 


\subsubsection{Electrical characterization of thin film transistors}

Since the majority of the carriers in organic materials are holes, most of the organic semiconductors exhibit p-type behavior. Models developed for inorganic semiconductors can be used to adequately describe the I-V characteristics of organic transistors. The typical output characteristics of a thin film transistor, which presents the dependence of the drain-source current $\left(\mathrm{I}_{\mathrm{DS}}\right)$ on the drain-source voltage $\left(\mathrm{V}_{\mathrm{DS}}\right)$ at different gate voltages is shown in Figure 3.11. For the linear operation regime, $\mathrm{I}_{\mathrm{DS}}$ increases linearly by increasing $\mathrm{V}_{\mathrm{DS}}$ and it can be determined using the following equation,

$$
I_{D S}=\frac{W C_{i}}{L} \mu\left(V_{G}-V_{T}-\frac{V_{D}}{2}\right) V_{D}
$$

where $C_{i}$ is the capacitance per unit area of the insulating layer, $W$ is the channel width, $L$ is the channel length, $\mathrm{V}_{\mathrm{T}}$ is the threshold voltage and $\mu$ is the field effect mobility. Mobility can be calculated by plotting $\mathrm{I}_{\mathrm{DS}}$ versus $\mathrm{V}_{\mathrm{G}}$ at a constant low drain voltage and fitting the data in the following equation:

$$
g_{m}=\left(\frac{\partial I_{D S}}{\partial V_{G}}\right)_{V_{D}=\text { const }}=\frac{W C_{i}}{L} \mu V_{D}
$$

By increasing the drain voltage, $\mathrm{I}_{\mathrm{DS}}$ will saturate which can be modeled by the following equation:

$$
I_{D S}=\frac{W C_{i}}{2 L} \mu\left(V_{G}-V_{T}\right)^{2}
$$

In the saturation regime, mobility can be calculated from the slope of the plot $\left(\mathrm{I}_{\mathrm{DS}}\right)^{0.5}$ versus $\mathrm{V}_{\mathrm{G}}$. Calculated values for the mobility in linear and saturation regime may be different. At low drain voltage or linear operation regime of the transistor, mobility can be affected by the 
contact problems, which result in the departure of the linear relationship between current and voltage. If the channel length (L) is comparable to the gate insulator thickness, current will not saturate by increasing the drain voltage and instead will show an upward trend at higher

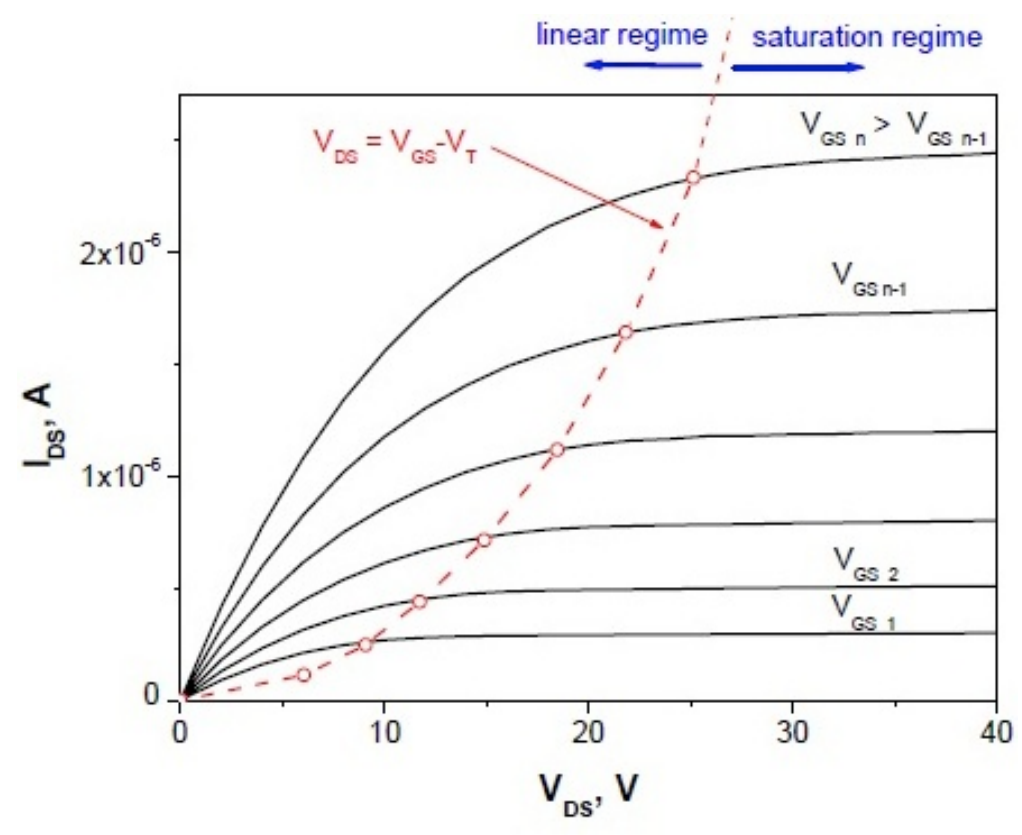
drain voltages. In this case, calculated mobility for these film transistor. devices will result in an enormously high value [4]. Charges have to be injected from the source electrode into the semiconductor in order to flow a current through the transistor channel. In other words, in an n-channel transistor, electrons should inject into the lower unoccupied molecular orbital (LUMO) level, and in a p-channel transistor, holes should inject into the highest occupied molecular orbital (HOMO) level of the organic semiconductor. In organic transistors, contrary to the case of silicon transistors, charge injection relies on the semiconductor-metal interface. This interface is treated as a Mott-Schottky barrier with the barrier height given as the difference between the values of the metal work function $\left(\varphi_{M}\right)$ and the semiconductor HOMO or LUMO level. If the work function of the metal contact is close to the HOMO or LUMO level of the semiconductor, a good Ohmic contact will be formed at the semiconductor-metal interface. Otherwise, a potential barrier is formed, leading to poor charge 
injection that introduces an extra resistance to the transistor. Considering this fact, a proper metal should be chosen to serve as the electrodes in order to obtain high performance devices.

Although aluminum is a well-known contact material used commonly in integrated circuits, its relatively low work function prevents its application in high performance p-channel OTFTs. Several researches have proven that by inserting a transition metal oxide layer between electrodes and organic semiconductors, high performance OTFTs can be achieved. It has been shown that the performance of OTFTs with the metal oxide buffer layer can be greatly improved over the transistors with $\mathrm{Al}$ as the drain and source electrodes. The contact barriers will be reduced by inserting this buffer layer. This layer also protects from diffusion or other chemical reactions between the organic layer and the metal contacts [67]. 


\section{CHAPTER 4: DEPOSITION TECHNIQUES}

\subsection{DEPOSITION TECHNIQUES}

\subsubsection{Overview}

For the past several decades, organic materials including polymers and small molecules have been of great interest for their various applications in the electronics and the semiconductors industry [32]. The main reasons for the popularity of organic electronics include high flexibility, low processing temperature, large area devices, light weight and availability of low-cost fabrication processes such as evaporation, spin-coating and printing [33]. A large number of studies have been focused on developing and optimizing deposition techniques for organic materials. The following section provides a summary of these techniques.

\subsubsection{Spin coating}

Spin coating is a fast and easy method to fabricate homogeneous organic thin films out of solutions. An excess amount of organic materials dissolved in a solution is placed and spread over a substrate by centrifugal force of the high speed rotation of the substrate. This method allows low-cost fabrication of organic electronic devices involving flexible and large area substrates. However, the fabrication of multilayered structures is very challenging using this technique. In addition, material waste and limited accuracy of thickness and uniformity of the films are the other disadvantages of this method. 


\subsubsection{Printing}

Low-cost and high-volume printed electronic devices can be fabricated on flexible substrates using printing techniques. Inkjet printing is one of the most commonly used printing techniques that provide a controlled deposition of organic solutions in specific locations of the substrate. Konarka demonstrated the first highly-efficient (with power conversion efficiency about 3\%) inkjet-printed organic bulk heterojunction solar cells in 2007 [68]. The devices were fabricated on ITO coated glass substrates. A thin layer $(60 \mathrm{~nm})$ of PEDOT:PSS was deposited by doctor blading on top of the ITO. Then samples were coated by a photovoltaic layer of P3HT:PCBM using a commercial piezoelectric driven inkjet printing tool from Fujifilm Dimatix, Inc. The schematic of the organic film formation by inkjet printing and the device structure are presented in Figure 4.1. This demonstration by Konarka confirms that organic solar cells can be fabricated using printing techniques, eliminating the requirement of clean room conditions as well as the high-temperature and high-vacuum processing environment of traditional semiconductor technologies.
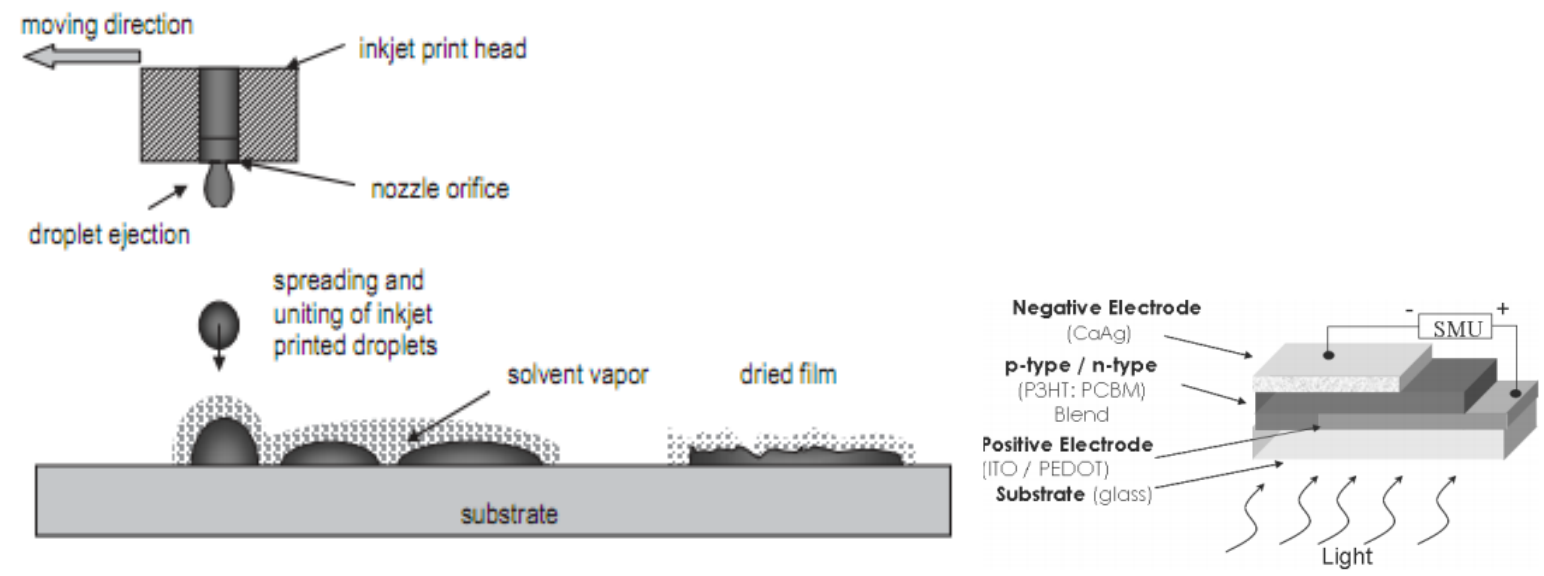

Figure 4.1 - Schematic of organic film formation by inkjet printing and the structure of the fabricated device (ITO/PEDOT:PSS/P3HT:PCBM/Ca:Ag) [33]. 


\subsubsection{Vacuum thermal evaporator}

Vacuum thermal evaporation (VTE) is another deposition technique in which organic materials are heated under vacuum condition. The evaporated material will be deposited onto the substrate which is placed several centimeters away from the source as shown in Figure 4.2. In this method, well-defined multilayer structures can be deposited without chemical interaction between different layers. However, relative lack of thickness and doping uniformity of the layers over large-area substrates are among the main problems that are present for the deposited films using this technique.

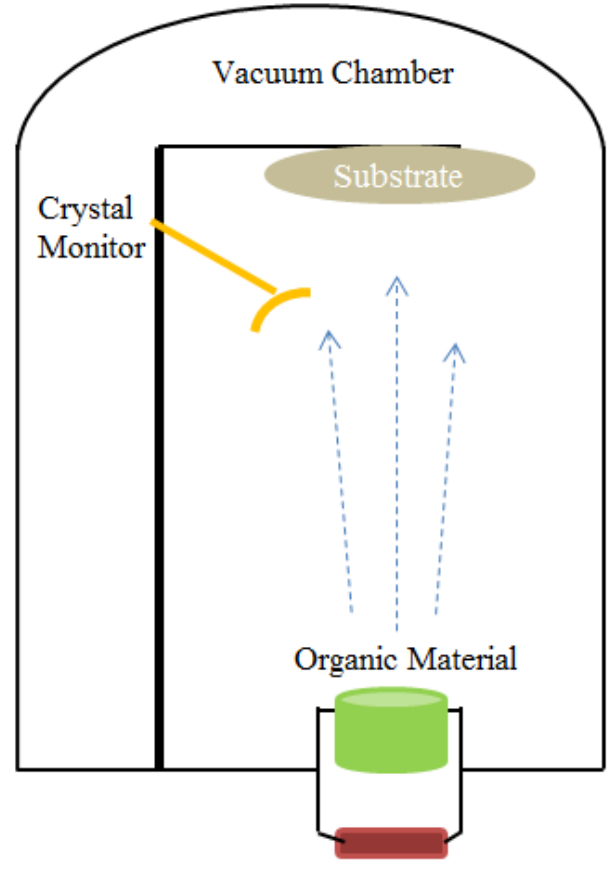

Voltage Supply

Figure 4.2 - Schematic of a Vacuum Thermal Evaporator (VTE) system.

In addition, contamination from the materials deposited on the wall of the chamber is another drawback of this method. In some cases, shadowing effect results in non-uniform deposition with holes spread over the films, which causes shorts in the device as well as increase in the device's series resistance.

\subsubsection{Organic vapor phase deposition (OVPD)}

Organic vapor phase deposition (OVPD) is another technique for deposition of organic materials. In this method, the scalability of printing techniques is combined with the ability of high purity and good control of organic materials deposition. In OVPD, a carrier gas will transport the thermally evaporated organic small molecules towards the substrate where 
condensation occurs (Figure 4.3). By adjusting the carrier gas flow rates, the relative concentrations of organic materials in the gas stream can be accurately controlled. Therefore, this method results in deposition of films with excellent uniformity and provides precise and high deposition rates and better morphology control.

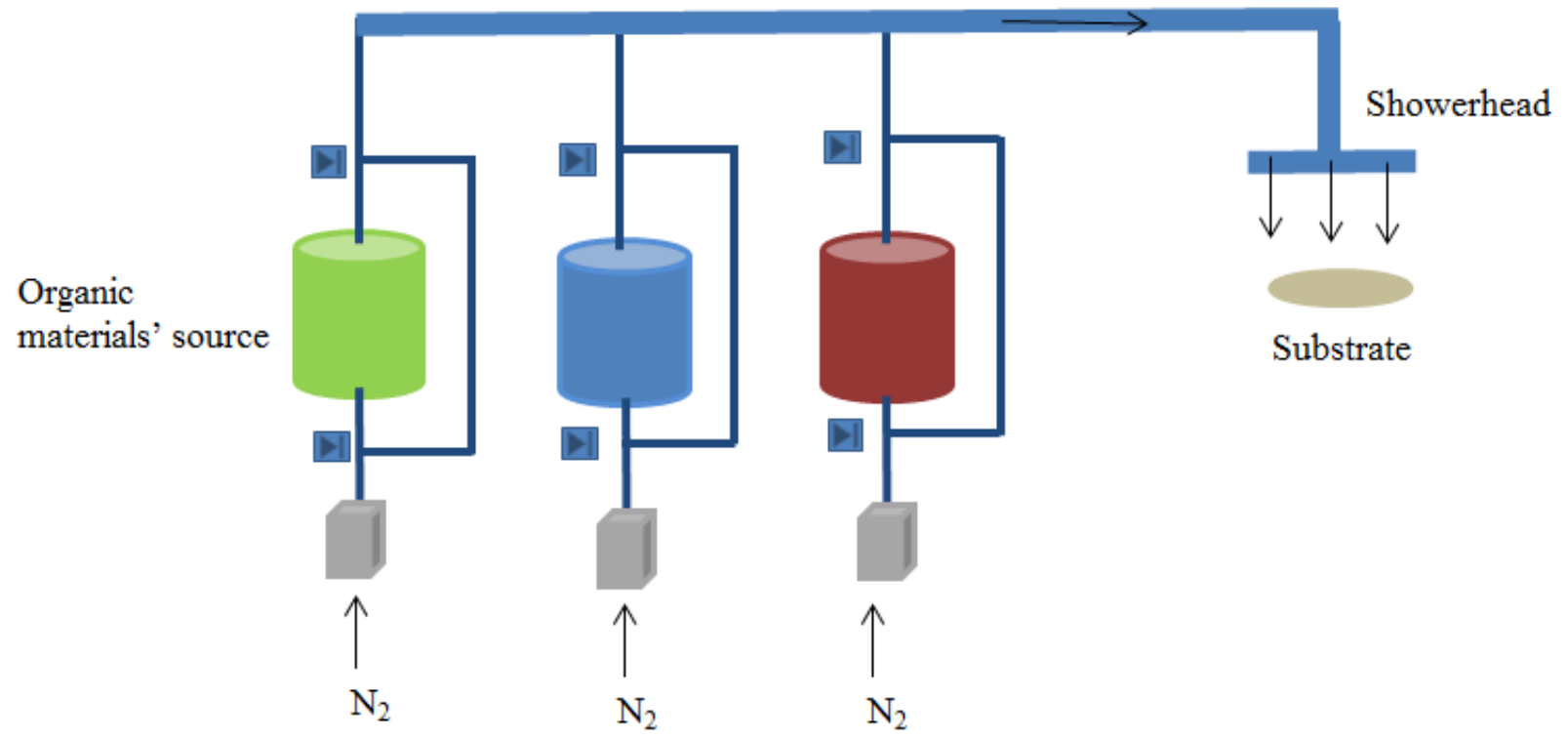

Figure 4.3 - Schematic of an Organic Vapor Phase Deposition (OVPD) system. 


\section{CHAPTER 5: CHARACTERIZATION TECHNIQUES}

\subsection{OVERVIEW}

Several material characterization techniques such as atomic force microscopy (AFM), Fourier transform infrared spectroscopy (FTIR), Raman spectroscopy, scanning electron microscopy (SEM), transmission electron microscopy (TEM) and X-ray diffraction (XRD) have been used to investigate the chemical and electrical properties of organic thin-films. AFM is a common technique that is used to study surface morphology and surface properties. However, this technique provides little if any information about the thin-film layer or the layers below the surface of a device.

Recently, a team of researchers at IBM has produced the first atomic force microscope (AFM) with a carbon monoxide tip. Using this new device, they have produced the first real images of a molecule of pentacene [69]. Figure 5.1 shows the AFM image of the pentacene crystal. In order to better investigate and understand the interaction between thin-film layers, FTIR and Raman techniques should be considered. However, FTIR spectroscopy has limitations in the type of material that can be used as the substrate. For

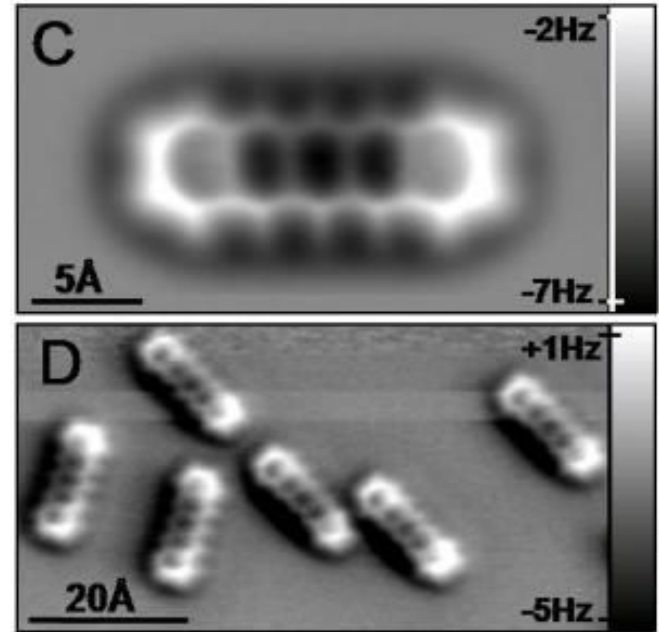

Figure 5.1 - Image of the pentacene molecule taken by AFM [69]. instance, since glass is not IR-transparent, IR radiation cannot penetrate deep enough into the glass, and hence, samples with glass substrate cannot be studied using FTIR spectroscopy. Raman spectroscopy is a high-resolution and flexible optical spectroscopy that is used to study 
the structural and electrical properties of materials. A large amount of data about molecular structure and the interaction between the metal and the organic layers at their surfaces can be obtained using Raman spectroscopy. Compared to FTIR, in Raman spectroscopy, the studied samples do not require any kind of preparation prior to scanning. Raman spectroscopy shows that the charge transport in the organic thin-film is determined by the structural qualities inside the grain boundaries and not the size or the amount of the grain boundaries $[9,11,12]$.

$\mathrm{XRD}$ is a non-destructive technique used to identify crystalline phases and orientation as well as to measure thickness of

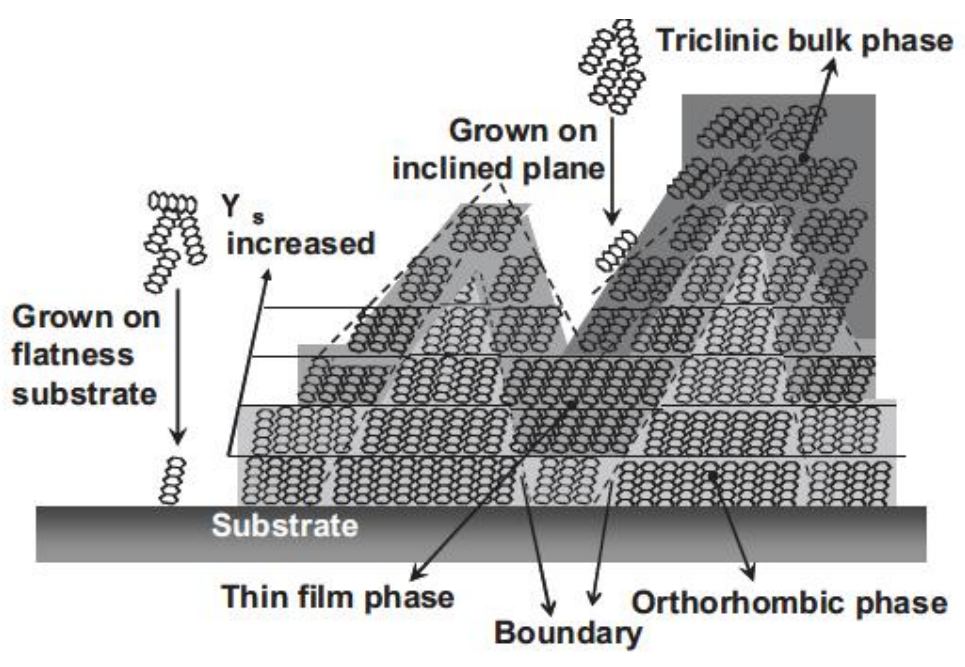

Figure 5.2 - Schematic evolutions of thin-film polymorphs of pentacene film with increasing thickness [12]. the thin films and multi-layers.

Additionally, it is used to determine atomic arrangement and structural properties such as lattice parameters, strain and grain size. XRD studies of pentacene thin films show an increment of the tilt angle $\left(\theta_{\text {tilt }}\right)$ of the pentacene molecule from the c-axis toward the a-axis by increasing the grown film thickness. The change of the tilt angle indicates that thin-film transformation behaviors are from the orthorhombic phase to the thin-film phase and then to the triclinic bulk phase [12]. These transition phases have been shown in Figure 5.2. SEM technique provides information about the external morphology, chemical composition and crystalline structure and orientation of the studied materials. SEM has been used to examine the surface morphology of 
the organic thin films and study the cross section of the fabricated organic transistors. TEM studies reveal that electrical properties of organic thin film are determined by the charge transport across the grain boundaries. In order to optimize the performance of the fabricated devices with organic small molecules as the active layer, grain boundaries structure and their dependence on the processing conditions should be carefully considered [9]. STEM and HRTEM are the two typical TEM imaging techniques used to investigate the molecular defect structures that can explain electrical and mechanical properties of the organic materials. In this work, AFM and X-ray reflectivity have been used to characterize the deposited organic films.

\subsection{ATOMIC FORCE MICROSCOPY}

The atomic force microscopy (AFM) is a very high resolution scanning probe microscopy method allowing imaging of any arbitrary surface. The principle of AFM operation is explained below. An atomically sharp tip is scanned over a surface and the feedback mechanism enables the piezo-electric scanners to maintain the tip either at a constant force to obtain height information, or at a constant height to obtain force information. Tips are typically made from $\mathrm{Si}_{3} \mathrm{~N}_{4}$ or $\mathrm{Si}$, and are placed at the end of a cantilever. Their radius of curvature is on the order of nanometers. When the tip is brought close to the sample surface, forces between the tip and the sample lead to the deflection of the cantilever according to Hooke's law $(\mathrm{F}=-\mathrm{kz})$, where $\mathrm{F}$ is the force, $\mathrm{k}$ is the stiffness of the lever and $\mathrm{z}$ is the distance the lever is bent. The force is calculated by measuring the deflection of the cantilever and knowing the stiffness of the cantilever. The deflection is measured using a laser spot reflected from the top of the cantilever into an array of photodiodes. A diode laser is focused onto the back of a reflective cantilever. As the tip scans the surface of the sample, moving up and down with the contour of the surface, the laser beam is 
deflected off the attached cantilever into a dual element photodiode. The photo-detector measures the difference in light intensities between the upper and lower photo-detectors, and then converts to voltage. Feedback from the photodiode difference signal, using software control from the computer, enables the tip to maintain either a constant force or height above the sample. In the constant force mode, the piezo-electric transducer monitors height deviation. In the constant height mode, the deflection force on the sample is measured. Contact and non-contact modes are the two common modes of AFM operation [70].

\subsubsection{Contact mode AFM}

As the name suggests, the tip and the sample remain in close contact during scanning. In this method, contact is defined as the repulsive regime of the inter-molecular force curve. The repulsive region of the curve lies above the $\mathrm{x}$-axis. The mean value of the repulsive force is $10^{-9} \mathrm{~N}$. This force is set by pushing the cantilever against the sample surface with a piezoelectric positioning element. The following figure explains the short force range

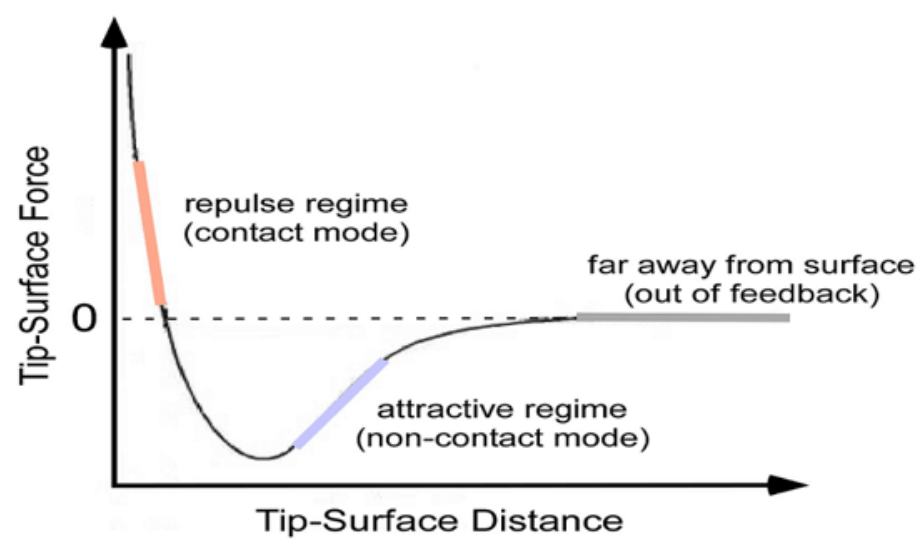

Figure 5.3 - Short force range in AFM [71].

in AFM. The deflection of the cantilever is sensed and compared to the desired values of deflection in a DC feedback amplifier. If the measured deflection is different from the desired value, the feedback amplifier applies a voltage to the piezo to raise or lower the sample relative to the cantilever to set the desired value of deflection. The voltage that the feedback amplifier 
applies to the piezo is a measure of the height of features on the sample and it is showed as a function of the lateral position of the sample. Problems with contact mode arise when excessive tracking forces are applied by the probe to the sample. The effects can be reduced by minimizing tracking force of the probe on the sample, but there are practical limits to the magnitude of the force. Under ambient conditions, sample surfaces are covered by a layer of adsorbed gases consisting primarily of water vapor and nitrogen. When the probe touches this contaminant layer, a meniscus forms and the cantilever is pulled by surface tension toward the sample surface. The magnitude of the force depends on the details of the probe geometry, but is typically on the order of 100 nano-N. The effect of this meniscus force and other attractive forces may be suppressed by operating with the probe and part or the entire sample totally immersed in liquid. There are many advantages to operating AFM with the sample and cantilever immersed in a fluid. These advantages include the elimination of capillary forces, the reduction of Van der Waals' forces

and the ability to study technologically or biologically important processes at liquid solid interfaces.

\subsubsection{Non-contact mode AFM}

In this mode, the tip is placed $50-150 \mathrm{~A}^{\circ}$ above the sample surface. Attractive Van der Waals forces acting between the tip and the sample are detected, and topographic images are obtained by scanning the tip above the surface. In the non-contact mode, the attractive forces from the sample are substantially weaker than the forces used by the contact mode. Therefore, the tip is given a small oscillation so that AC detection methods can be used to detect the small forces between the tip and the sample. By measuring the change in the amplitude, phase or frequency of the oscillating cantilever in response to force gradients from the sample, the force 
can be measured. For highest resolution, it is necessary to measure force gradients from Van der Waals forces which may extend only a nanometer from the sample surface. Generally, the fluid contaminant layer is substantially thicker than the range of the Van der Waals force gradient. As the oscillating probe becomes trapped in the fluid layer or hovers beyond the effective range of the forces, attempts to image the true surface with non-contact AFM do not succeed.

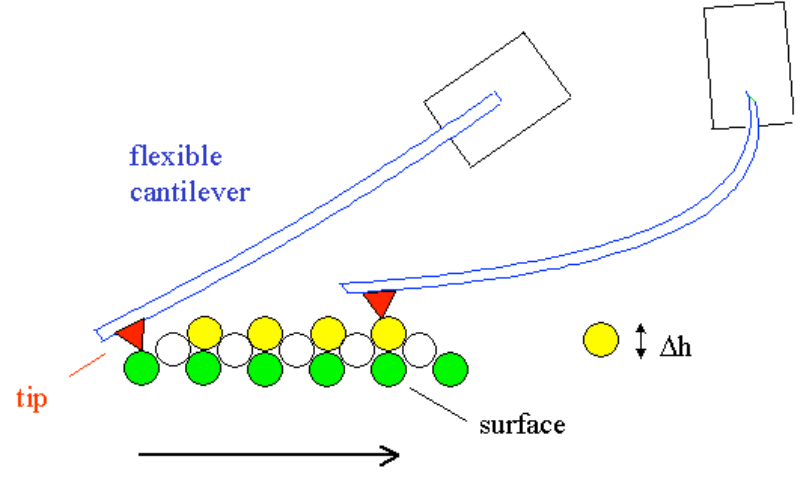

a) Contact mode

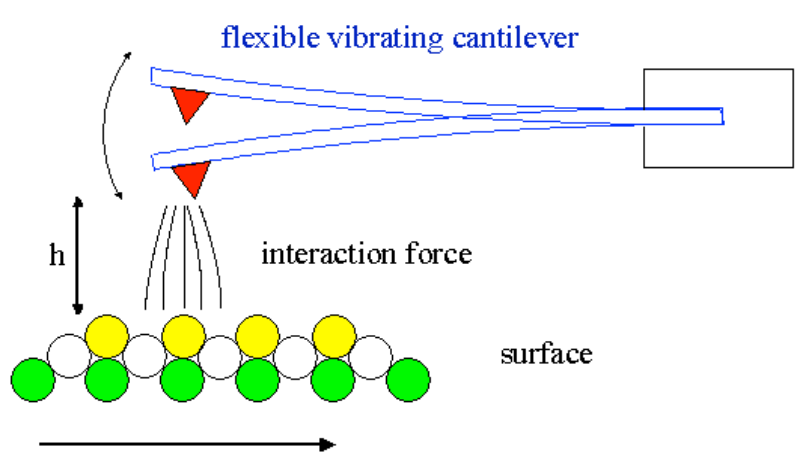

horizontal sample motion

b) Non-contact mode

Figure 5.4 - Schematic of contact and non-contact mode AFM [72].

\subsubsection{Tapping Mode AFM}

This technique allows high resolution topographic imaging of sample surfaces that are easily damaged, loosely held to their substrate or difficult to image by other AFM techniques. Tapping mode overcomes problems associated with friction, adhesion, electrostatic forces and other difficulties observed in the conventional AFM scanning methods by alternately placing the tip in contact with the surface to provide high resolution and then lifting the tip off the surface to avoid dragging the tip across the surface. Tapping mode imaging is implemented in ambient air by oscillating the cantilever assembly at or near the cantilever's resonant frequency. When the tip is not in contact with the surface, the motion causes the cantilever to oscillate with high amplitude. 
The oscillating tip is then moved toward the surface until it begins to lightly touch the surface. During scanning, the vertically oscillating tip alternately contacts the surface and lifts off. As the oscillating cantilever begins to intermittently contact the surface, the cantilever oscillation is reduced due to the energy loss caused by the tip contacting the surface. Surface features can be identified and measured by observing the reduction in oscillation amplitude. During tapping mode operation, the cantilever oscillation amplitude is maintained constant by a feedback loop. Selection of the optimal oscillation frequency is software-assisted and the force on the sample is automatically set. When the tip passes over a bump in the surface, the cantilever has less room to oscillate and the amplitude of oscillation decreases. Conversely, when the tip passes over a depression, the cantilever has more room to oscillate and the amplitude of oscillation increases. The oscillation amplitude of the tip is measured by the detector and then the digital feedback loop adjusts the tip sample separation to maintain constant amplitude. Operation in the frequency mode operation can also be done. Frequency mode operation is more sensitive and allows the use of very stiff cantilevers. In this method, the change in the oscillation frequency provides the information about the surface. AFM tips also play an important role in the resolution of topographic imaging. Greater resolution can be achieved by using a sharp tip. The best tips may have a radius of curvature of approximately $5 \mathrm{~nm}$. The degree of sharpness of a tip is described in terms of tip convolution.

\subsection{X-RAY DIFFRACTION}

X-ray diffraction discovered by Max von Laue in 1912 has become a well-established technique to probe the structural arrangement of atoms and molecules in materials. The wavelength scale of X-rays is in the range of an Angstrom $\left(10^{-10} \mathrm{~m}\right)$ which is comparable to the 
size of atoms. This makes X-ray scattering a powerful method for determining ordered atomic structures. The next section provides a short overview of this technique but a more detailed description can be found in the references [73-75].

\subsubsection{Basic principle of $\mathrm{X}$-ray diffraction}

An X-ray beam can be considered as a monochromatic plane wave in the following form,

$$
\vec{E}(\vec{r}, t)=\vec{\varepsilon} E_{0} e^{i(\vec{k} \cdot \vec{r}-w t)}
$$

where $\hat{\varepsilon}$ is the polarization of the electric field written as a unit vector and $\vec{k}$ is the wave vector where $\hat{\varepsilon} \cdot \vec{k}=0$. An X-ray photon can either be scattered or absorbed when it interacts with a medium. The scattering process involves both the incident wave $\left(\vec{k}_{\mathrm{i}}\right)$ and the scattered wave $\left(\vec{k}_{\mathrm{f}}\right)$. Momentum transfer, $\vec{q}$ can be defined as $\vec{q}=\vec{k}_{i}-\vec{k}_{f}$. We will discuss these processes for an electron, an atom, a molecule and a crystal structure.

\subsubsection{One electron scattering}

As an electron interacts with an X-ray beam, it will be forced to vibrate and radiates as a second source (Figure $5.5(\mathrm{a}))$. The magnitude $\left(\vec{E}_{\text {rad }}(\vec{R}, t)\right)$ and the intensity (I) of the radiated field can be described as follows,

$$
\vec{E}_{r a d}(\vec{R}, t)=-r_{e} \frac{e^{-i \vec{k} \cdot \vec{R}}}{|R|} \cos 2 \theta \cdot \vec{E}_{i n}
$$

where $\vec{R}$ is the distance from the scattering point to an observation point $\mathrm{X}, 2 \theta$ is the angle with respect to the direction of the incident beam and $r_{e}$ is the Thomson scattering length. 


$$
\begin{aligned}
& r_{e}=\frac{q^{2}}{4 \pi \varepsilon_{0} m c^{2}}=2.8 \times 10^{-5} A^{\circ} \\
& I \propto\left|E_{\text {rad }}\right|^{2}=\left(\frac{r_{e}^{2}}{R^{2}}\right)\left\langle E_{\text {in }}^{2}\right\rangle P
\end{aligned}
$$

where $\mathrm{P}$ is the polarization factor which equals to 1 or $\cos ^{2} 2 \theta$ for synchrotron radiation depending on the scattering plane and is equal to $\frac{1}{2}\left(1+\cos ^{2} 2 \theta\right)$ for an unpolarized source.

\subsubsection{One atom scattering}

For an atom containing $\mathrm{Z}$ electrons, the distribution of the electrons can be considered continuously and described by the electron density function $\rho(\mathrm{r})$. The superposition of the charge distribution inside the atom will define the scattered radiation field. As shown in Figure 5.5 (b), the phase difference of the incident wave as it interacts with the volume element at the origin $\mathrm{O}$ and the position $\vec{r}$ should be considered in order to evaluate this superposition. This phase difference can be described as follows,

$$
\Delta \emptyset(\vec{r})=\left(\vec{K}-\overrightarrow{K^{\prime}}\right) \cdot \vec{r}=\vec{Q} \cdot \vec{r}
$$

where $\vec{Q}$ is the wave vector transform or scattering vector and equals $2 \vec{K} \sin \Theta=(4 \pi / \lambda) \sin \Theta$ for elastic scattering. The contribution of the volume element $\mathrm{d} \vec{r}$ at $\vec{r}$ to the scattering field is $-\mathrm{r}_{\mathrm{e}}$ $\rho(\vec{r}) \mathrm{d} \vec{r}$ with the phase factor of $e^{\mathrm{i} \vec{Q} \cdot \vec{r}}$. Therefore, the total scattering length of the atom can be described as the Fourier transform of the distribution of the electrons in the atom as described in Equation 5.6.

$$
-r_{e} f^{0}(\vec{Q})=-r_{e} \int \rho(\vec{r}) e^{\mathrm{i} \vec{Q} \cdot \vec{r}} d \vec{r}
$$


where $f^{0}(\vec{Q})$ is known as atomic form factor. If all of the volume elements scatter in phase, $f^{0}(\vec{Q})$ equals the number of electrons in the atom. As the volume elements start scattering out of phase, $\vec{Q}$ increases and $f^{0}(\vec{Q})$ will become zero.

\subsubsection{One molecule scattering}

As illustrated in Figure 5.5, a molecule is composed of a number of atoms. Therefore, the form factor of the molecule can be determined by considering the atomic form factors of the consisting atoms (labeled as $\mathrm{j}$ atom) and their positions inside the molecule (Equation 5.7),

$$
f^{m o l}(\vec{Q})=\sum_{j} f_{j}(\vec{Q}) \cdot e^{\mathrm{i} \vec{Q} \cdot \vec{r}_{j}}
$$

where $f_{j}(\vec{Q})$ is the atomic form factor of the $\mathrm{j}^{\text {th }}$ atom in the molecule.

\subsubsection{A crystal scattering}

The interaction of the incident X-rays with the crystalline material which is periodic in space will produce constructive interference under Bragg's law condition $(\mathrm{n} \lambda=2 \mathrm{~d} \sin \theta)$. This relates the wavelength of the X-ray $(\lambda)$ to the diffraction angle $(\theta)$ and the lattice spacing in a crystalline sample (d) (Figure 5.5 (c)).
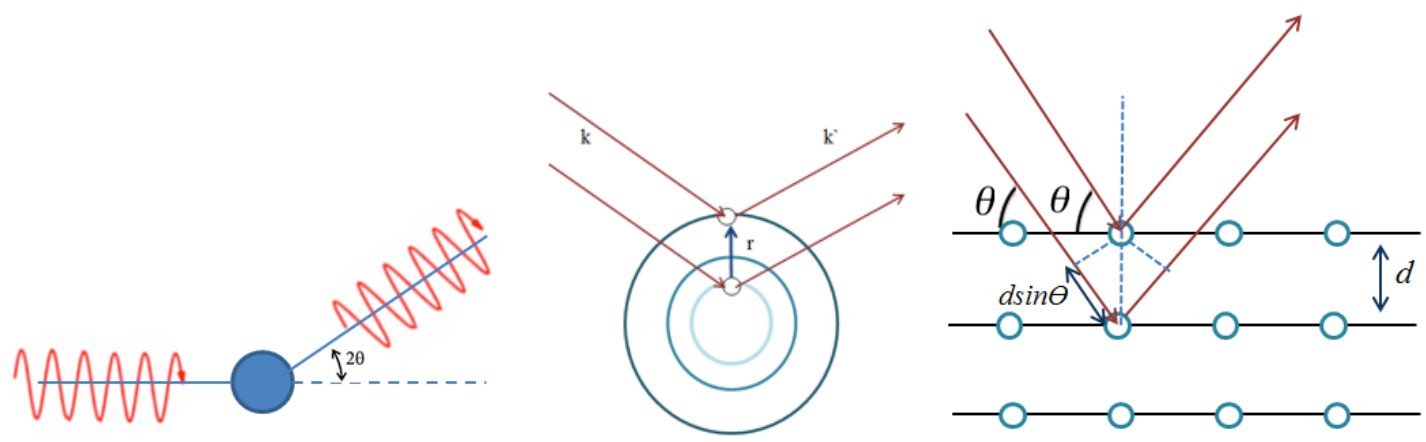

Figure 5.5 - The X-ray scattering from (a) an electron, (b) one atom and (c) a crystal. 


\subsection{X-RAY REFLECTIVITY}

As the X-rays interact with different media, there will be some refraction at the interfaces due to different refractive indices (Snell's law). Apart from the refracted beam, there will also be a reflected part and in order to describe the refraction and the reflection phenomena for X-rays, a refractive index of $\mathrm{n}$ can be introduced as $\mathrm{n}=1-\delta+\mathrm{i} \beta$, where $\delta$ and $\beta$ are the dispersion and absorption of the material, respectively. Typically, for solid materials $\delta$ is in the order of $10^{-5}$ and $\beta$ is usually two orders of magnitude smaller than $\delta$. When X-rays are irradiated on to the sample at very low angles, there is total reflection of X-rays from the sample surface. For small angles of radiation, the refractive index is very close to unity and the electron density can be considered as continuous. In the small angle range, reflection can be defined using the classical reflection of the electromagnetic waves which yield the expression for the Fresnel reflectivity. Following equations are the reflectivity and transmittivity amplitudes,

$$
r=\frac{k_{i, z}-k_{t, z}}{k_{i, z}+k_{t, z}} \text { and } t=\frac{2 k_{i, z}}{k_{i, z}+k_{t, z}}
$$

where $k_{i, z}$ and $k_{t, z}$ are the vertical components of the incident and transmitted waves, respectively. If the medium possesses regions with different electron densities, then the boundary conditions of the electromagnetic fields at each interface should be applied when calculating the reflectivity. In this case, shown in Figure 5.6, the X-ray reflectivity can be derived considering a multilayered structure consisting of $\mathrm{n}$ layers with $(\mathrm{n}+1)$ interfaces. Using Parratt formalism [76], the transmitted X-ray in the top-most layer serves as a new source for the scattering at the next lower interface. The Fresnel reflection coefficient of interface $\mathrm{j}$ can be calculated as 


$$
r_{j}=\frac{k_{j, z}-k_{j+1, z}}{k_{j, z}+k_{j+1, z}}
$$

The intensity of the X-ray reflectivity at interface $\mathrm{j}$ can be calculated using the recursive algorithm from the ratio between reflection and transmission at the interfaces $\mathrm{j}$ and $\mathrm{j}+1$, as shown in the following equation,

$$
x_{j}=\frac{r_{j}}{t_{j}}=e^{-2 i k_{z, j} z_{j}} \frac{r_{j, j+1}+X_{j+1} e^{2 i k_{z, j+1} z_{j}}}{1+r_{j, j+1} X_{j+1} e^{2 i k_{z, j+1} z_{j}}}
$$

assuming no reflection from the substrate $\left(\mathrm{X}_{\mathrm{n}+1}=0\right)$ and total transmission for the vacuum layer $\left(T_{1}=1\right)$. For real surfaces, in order to account for the roughness, a term of $e^{-2 k_{z, j} k_{z, j+1} \sigma_{j, j+1}^{2}}$ should be included into each Fresnel reflection coefficient.

As described in Figure 5.7, the reflected x-rays from different interfaces give rise to interference fringes. Additional peaks (called Bragg peaks) will be observed in the reflectivity pattern, provided that the thin film is a layered film with periodic electron density (Figure 5.7 (c)) and the
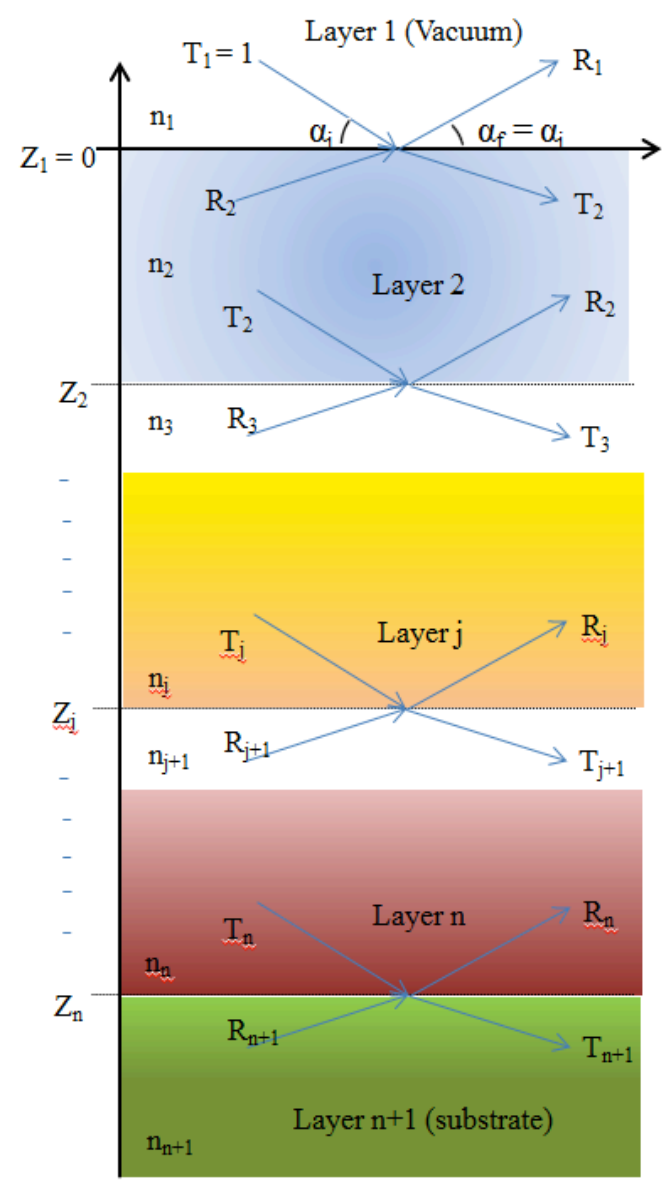

Bragg condition is fulfilled $(2 \mathrm{~d} \sin \theta=\mathrm{n} \lambda)$. For real surfaces with roughness $\sigma$, intensity

Figure 5.6 - Schematic of the reflection for a film with a multilayered structure. 
will decrease as shown in Figure 5.7 (d). Total thickness of a thin film can be calculated based on the periodicity of the low angle fringes. Interface and surface roughness can be derived from the damping of the intensity of the reflectivity pattern.

a)

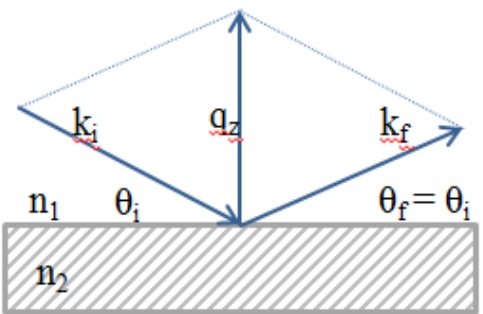

b)

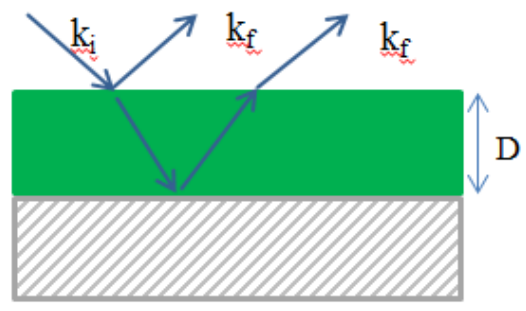

c)
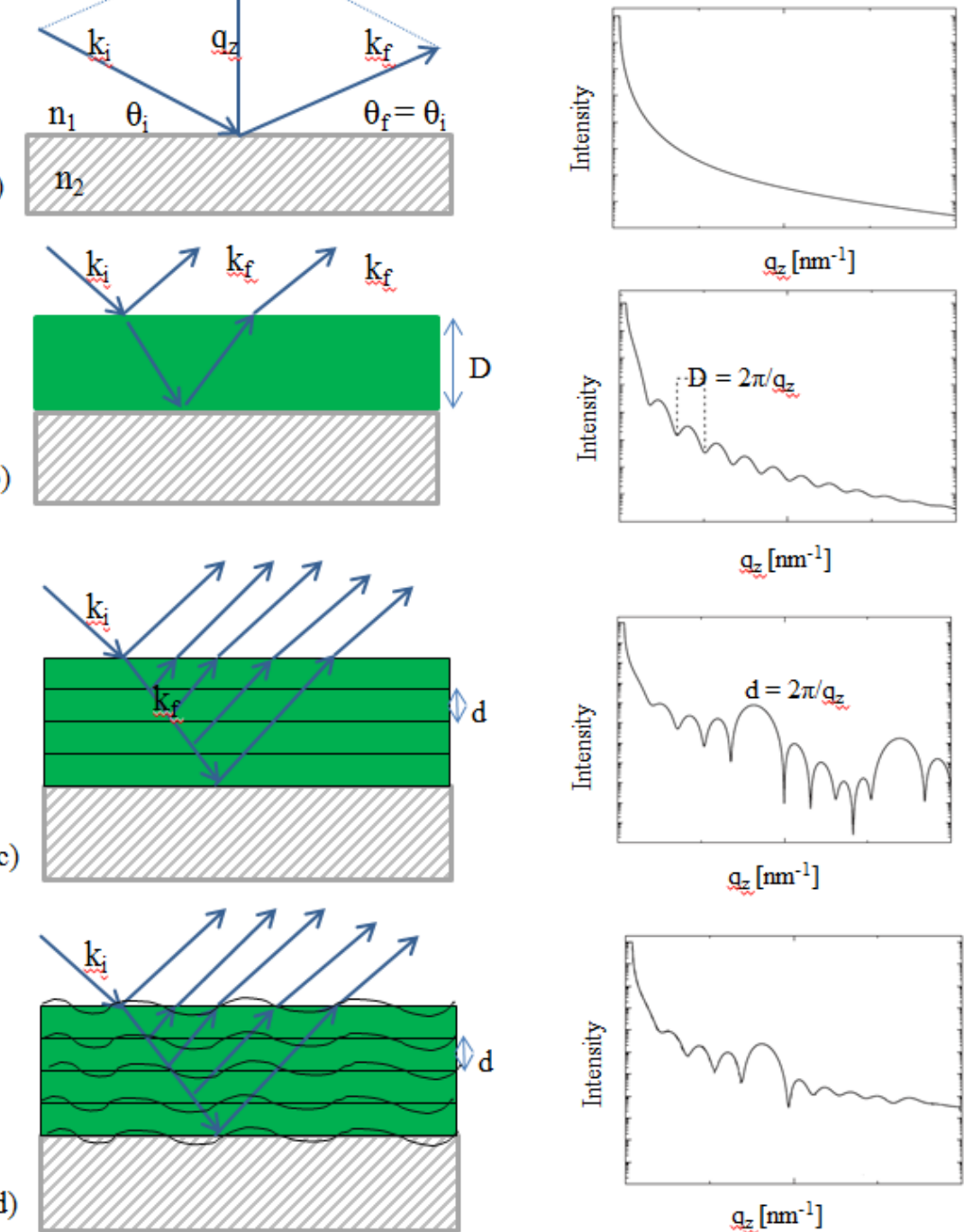

Figure 5.7 - Reflectivity patterns as a function of momentum transfer for (a) a smooth vacuum/medium interface, (b) a film with two interfaces, (c) a film with a periodic electron density and (d) a film same as previous film but considering interface and surface roughnesses. 


\subsection{DEPOSITION METHOD AND EQUIPMENT}

In this work, all of the thin films and organic devices have been fabricated using the vacuum thermal evaporation technique. Several fabrication processes have been designed and executed using an academic series research evaporator from Trovato Mfg., Inc. as shown in Figure 6.1. We have maintained and troubleshooted our system by adhering to several protocols listed below:

- Monitor pressure versus time during the pump down of the chamber from the atmospheric pressure to a high vacuum (less than $4 \times 10^{-7}$ Torr)

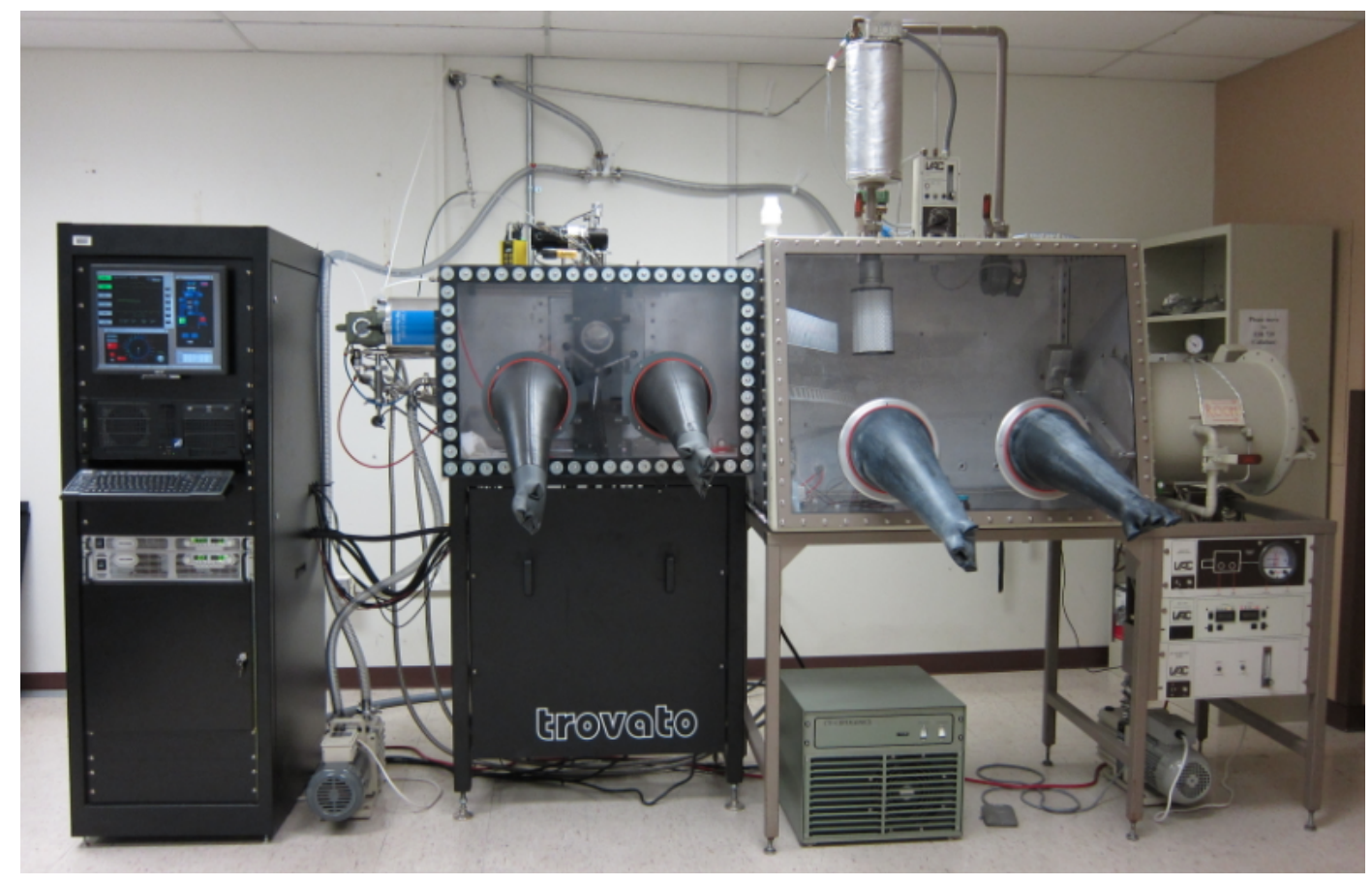

Figure 6.1 - The academic series research evaporator from Trovato Mfg., Inc with the attached glove box. 
- Perform regular cryopump regeneration

- Develop and perform cleaning process of the chamber

- Perform regular glove box regeneration

As shown in Figure 6.2, the thermal evaporator system used in this research has 8 sources with 4 crystal monitors, one for each two adjacent sources. For each material deposited in this system, a relevant film and process have been developed by defining the applied power, the values for the proportional-integral-derivative controller (PID controller) and the deposition rate. The proper boat and crucible for depositing each material have been determined through several experiments. The metal oxides and organic materials have been deposited using quartz boats with Tungsten crucibles. For metal deposition boats made of Boron-Nitride have been used. In order to pattern the fabricated devices, several

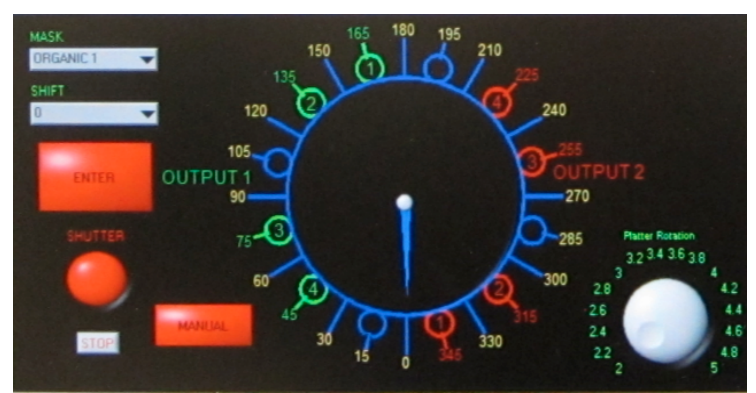

Figure 6.2 - Schematic of different parts of the thermal evaporator system.

sets of metal shadow masks have been designed and made using stainless steel.

For material characterization part of this work, different thicknesses of several thin films of organic materials have been deposited using the abovementioned vacuum thermal evaporator 
system. Moreover, CuPc/PTCDI-C8 films with different structures such as bilayer, layered and co-deposited as well as LiF/PTCDI-C8 and LiF/Pentacene bilayers have been prepared and characterized. For device characterization part of this study, organic solar cells and organic thin film transistors have been prepared using the following fabrication processes.

\subsection{DEVICE FABRICATION PROCESS}

Organic solar cells studied in this work were fabricated by thermally evaporating two organic materials $\mathrm{CuPc}$ and PTCDI-C8 as the donor and acceptor layers, respectively. $\mathrm{MoO}_{3}$ and $\mathrm{Alq}_{3}$ were used as the hole and electron transport layers, respectively. Indium-tin-oxide (ITO) coated glasses with a sheet resistance of approximately $15 \Omega / \square$ were used as the substrate for the fabricated cells. They were cleaned using acetone, isopropyl alcohol and de-ionized water in an ultrasonic cleaner, then dried by nitrogen and treated by oxygen plasma. A schematic of this process is presented in Figure 6.3.

Several cells with various thicknesses of organic layers ranging from 20 to $60 \mathrm{~nm}$ and different $\mathrm{MoO}_{3}$ and $\mathrm{Alq}_{3}$ thicknesses were fabricated. For all of the devices, a $75 \mathrm{~nm} \mathrm{Al}$ layer was deposited as the cathode

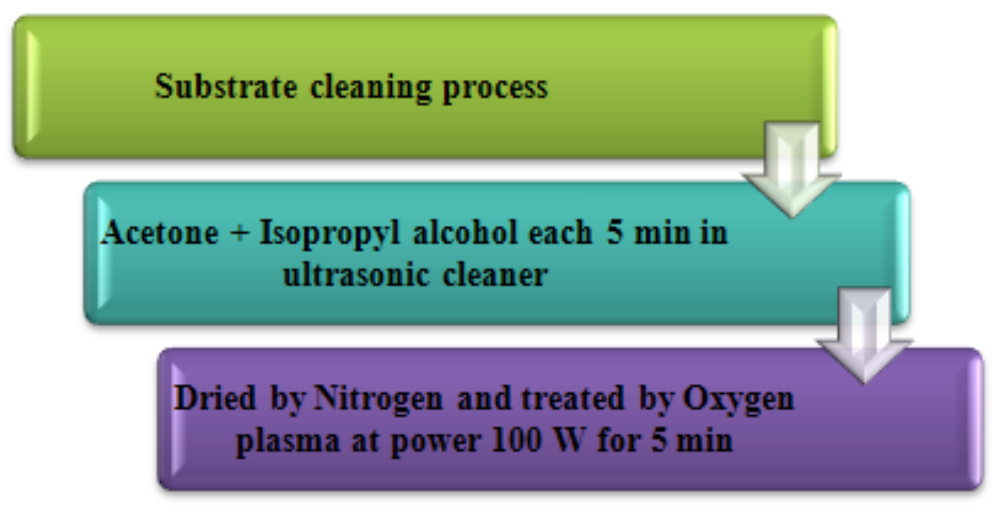

Figure 6.3 - The substrate cleaning process used in this work.

electrode using a metal shadow

mask. The fabrication process for the solar cells as well as the schematic structure of the fabricated devices is shown in Figure 6.4 (a) and Figure 6.5 (a). 
Furthermore, planar organic thin film transistors were fabricated with top contact geometry. The schematic structure of the fabricated device is presented in Figure 6.5 (b). Fabricated transistors have a structure similar to the conventional inorganic metal-oxidesemiconductors, with one principal difference in the use of lithium fluoride (LiF) instead of oxide as the gate insulating layer. The fabrication process is as follows. An aluminum (Al) layer with a thickness of $100 \mathrm{~nm}$ was deposited onto a pre-cleaned glass substrate as the gate electrode. After gate electrode deposition, a $120 \mathrm{~nm}$ layer of $\mathrm{LiF}$ was deposited as the gate dielectric layer, followed by deposition of $100 \mathrm{~nm}$ of pentacene or PTCDI-C 8 as the active layer. Finally, vanadium pentoxide $\left(\mathrm{V}_{2} \mathrm{O}_{5}\right)$ and $\mathrm{Al}$ were deposited onto the organic cell surface through

(a)

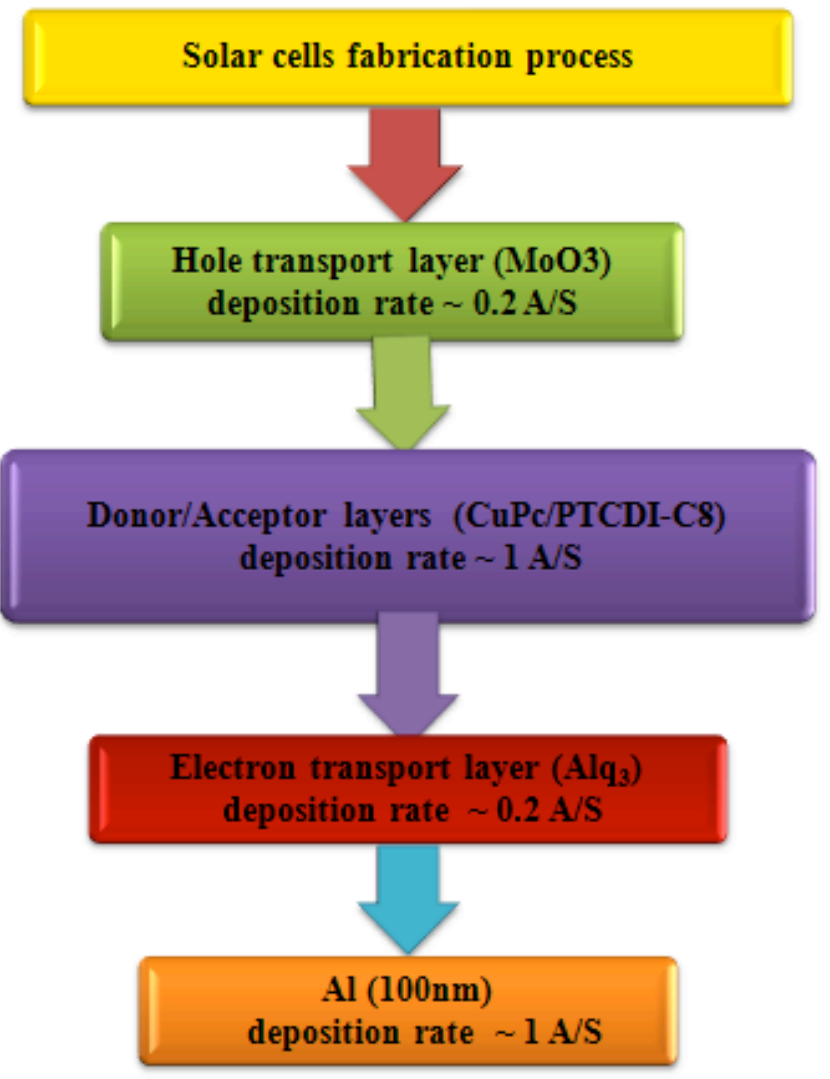

(b)

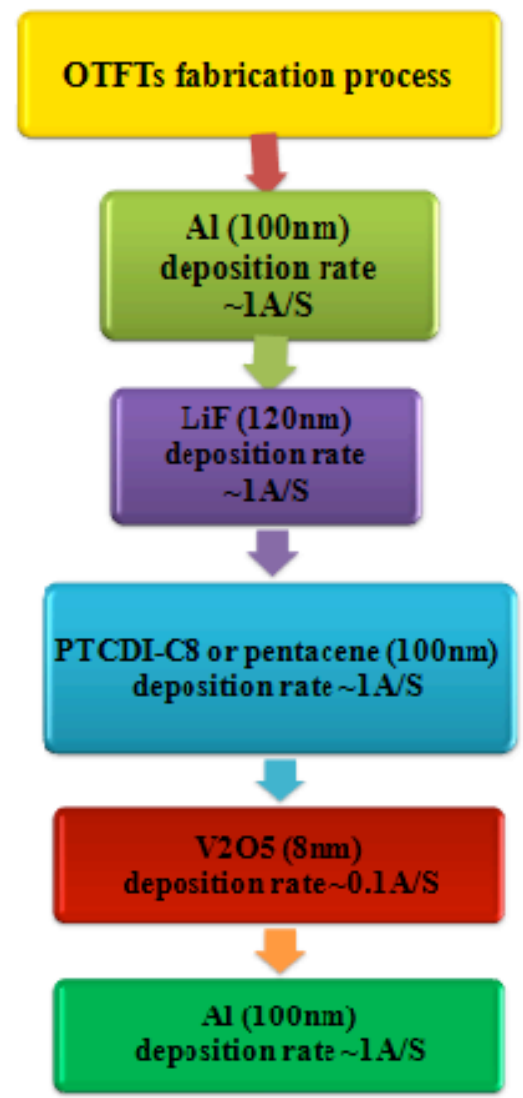

Figure 6.4 - The fabrication process used for (a) organic solar cells and (b) organic transistors. 
a shadow mask to form the source and the drain electrodes. The thickness of the $\mathrm{V}_{2} \mathrm{O}_{5}$ and $\mathrm{Al}$ films were $8 \mathrm{~nm}$ and $100 \mathrm{~nm}$, respectively. The $\mathrm{V}_{2} \mathrm{O}_{5}$ layer serves as the thin insulating buffer layer to modify the organic-metal barrier and facilitates the charge injection. The fabrication process for the transistors is presented in Figure 6.4 (b).

All of the organic materials and metal oxides were purchased from Sigma Aldrich with the highest grade of purity and used without further treatments. All of the layers were deposited in a thermal evaporator system with a pressure of less than $4 \times 10^{-7}$ Torr and without heating the substrate. Fabricated cells were characterized under ambient condition without any encapsulation. Electrical measurements were taken using an Agilent (4155C) semiconductor parameter analyzer and a Xenon lamp with AM1.5 filter. The light intensity of the lamp (100 $\mathrm{mW} / \mathrm{cm}^{2}$ ) was measured by a calibrated silicon detector.
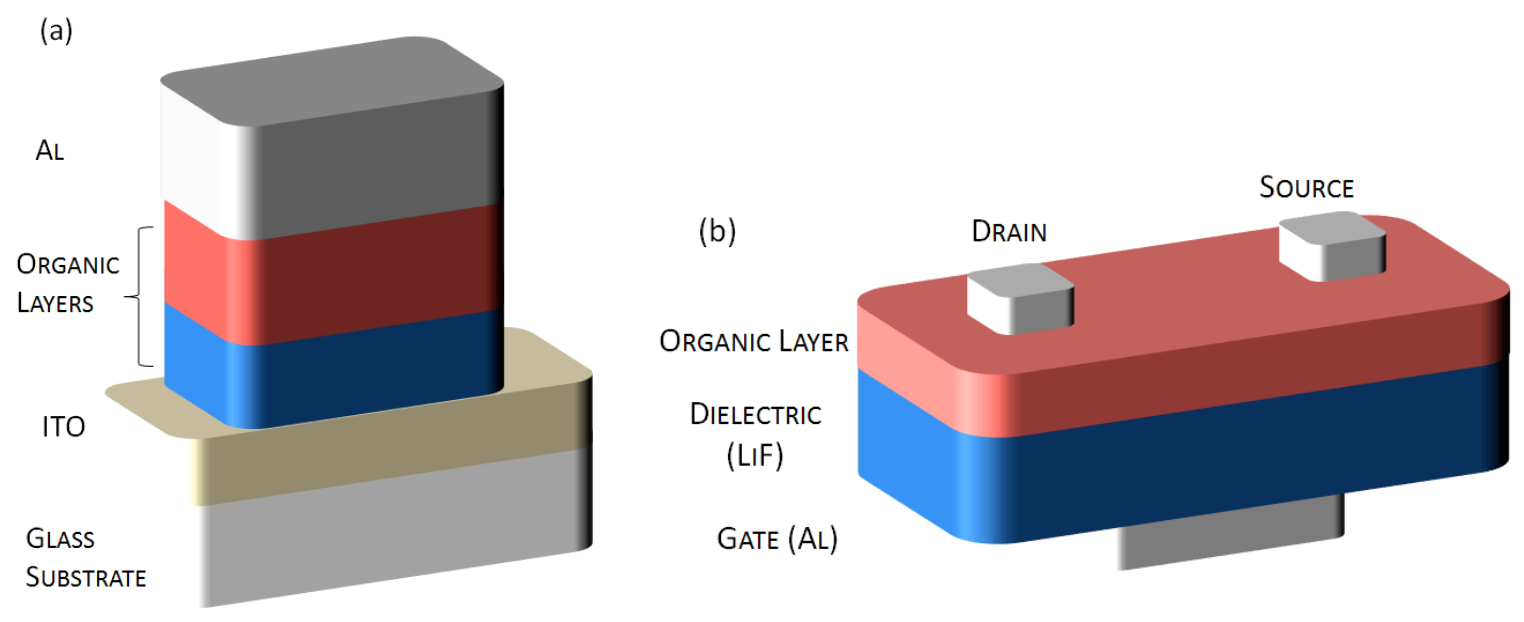

Figure 6.5 - Schematic diagram of the fabricated (a) solar cells with the following structure: $\left(\mathrm{ITO} / \mathrm{MoO}_{3} / \mathrm{CuPC} / \mathrm{PTCDI}-\mathrm{C} 8 / \mathrm{Alq}_{3} / \mathrm{Al}\right)$ and (b) transistors with the top contact geometry. 


\subsection{OVERVIEW}

Several sets of samples with thicknesses ranging from 20 to $45 \mathrm{~nm}$ were deposited on ptype boron-doped Si (111) substrates with native oxide using a thermal organic evaporator. The film deposition was performed under high vacuum conditions with a base pressure of approximately $10^{-7}$ Torr at a rate of $1 \AA / \mathrm{sec}$. Spectroscopic ellipsometry measurements were performed using a JA Wollam M-2000V Ellipsometer in the spectral range from 1.4 to $5.2 \mathrm{eV}$ with a resolution of around $1 \mathrm{meV}$, and at an angle of incidence of $70^{\circ}$. The absorption spectra of the PTCDI-C8 films were investigated in the wavelength range of 300 to $1100 \mathrm{~nm}$ using a UVVIS-NIR spectrophotometer (Perkin-Elmer Lambda 45). Topographic images were taken using a Veeco Multimode Scanning Probe Microscope AFM operated in noncontact mode. X-ray reflectivity was performed using a Bruker-AXS D8-Discover high resolution X-ray diffractometer. This system utilizes a horizontal goniometer, fixed X-ray tube with a $\mathrm{Cu}$ anode ( $\mathrm{CuK} \alpha$ radiation, $\lambda=0.15418 \mathrm{~nm})$, Göbel mirrors, $\mathrm{Cu}$ rotary absorber, 4-bounce $\mathrm{Ge}(022)$ monochromator and Lynx Eye position sensitive detector (PSD) with automated Iris. The reflectivity data were analyzed using the Parratt formalism which takes into account multiple scattering effects. All of the experiments were carried out in the ambient conditions. A summary of the material characterization is presented in the next sections.

\subsection{ATOMIC FORCE MICROSCOPY (AFM) - ROUGHNESS}

AFM in tapping mode was used to collect information about the morphology and structure of the PTCDI-C8 thin films. Several scan sizes from 0.5 to $5 \mu \mathrm{m}$ on different film 
thicknesses were performed which revealed that PTCDI-C8 forms smooth layered films. The following figures show AFM scans of ITO substrates annealed in nitrogen for $10 \mathrm{~min}$ at $300{ }^{\circ} \mathrm{C}$ and silicon substrates.

The root mean square roughness $(\sigma)$ of the ITO substrates is approximately $3 \mathrm{~nm}$ (Figure 7.1 (a)) and of the silicon substrate is approximately $0.6 \mathrm{~nm}$ (Figure 7.1 (b)). Films with different thicknesses of PTCDI-C8 were deposited on these substrates at room temperature and their AFM images are presented in Figures 7.2 and 7.3. Comparing AFM images of PTCDI-C8 on ITO with the silicon substrate, it can be seen that the morphology of the substrate has affected the
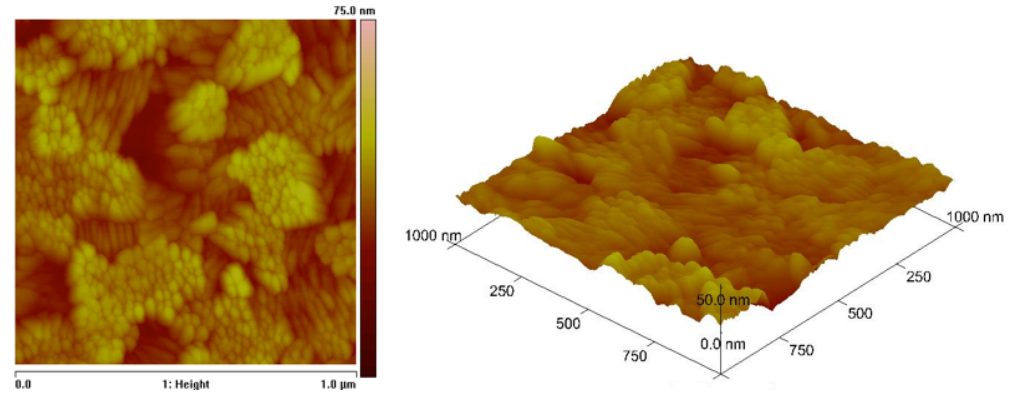

structure and the morphology of
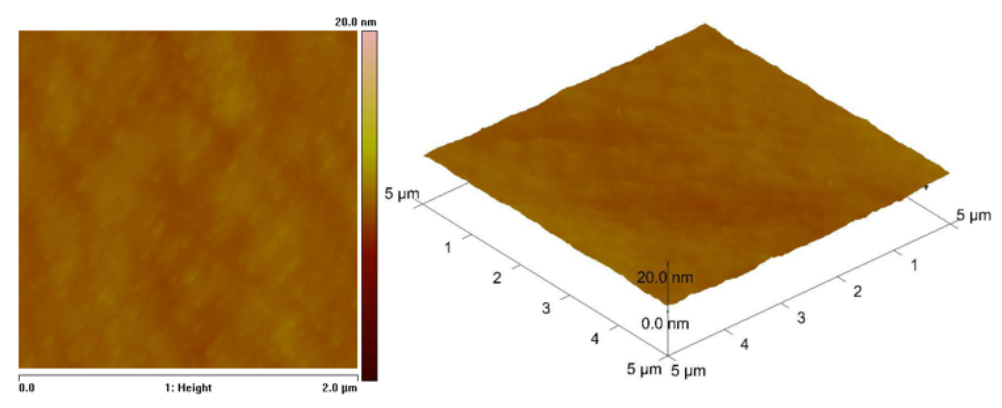

the deposited films. Observed

Figure 7.1 - AFM images of (a) ITO substrates annealed at $500{ }^{\circ} \mathrm{C}$ in nitrogen for $10 \mathrm{~min}$, and (b) silicon substrates with native silicon dioxide.

terraces in the ITO substrates are propagated through the deposited PTCDI-C8 film. In addition, for thin films of PTCDI-C8 on ITO substrate, larger roughness was observed compared to the films deposited on silicon substrate with smoother surface. 


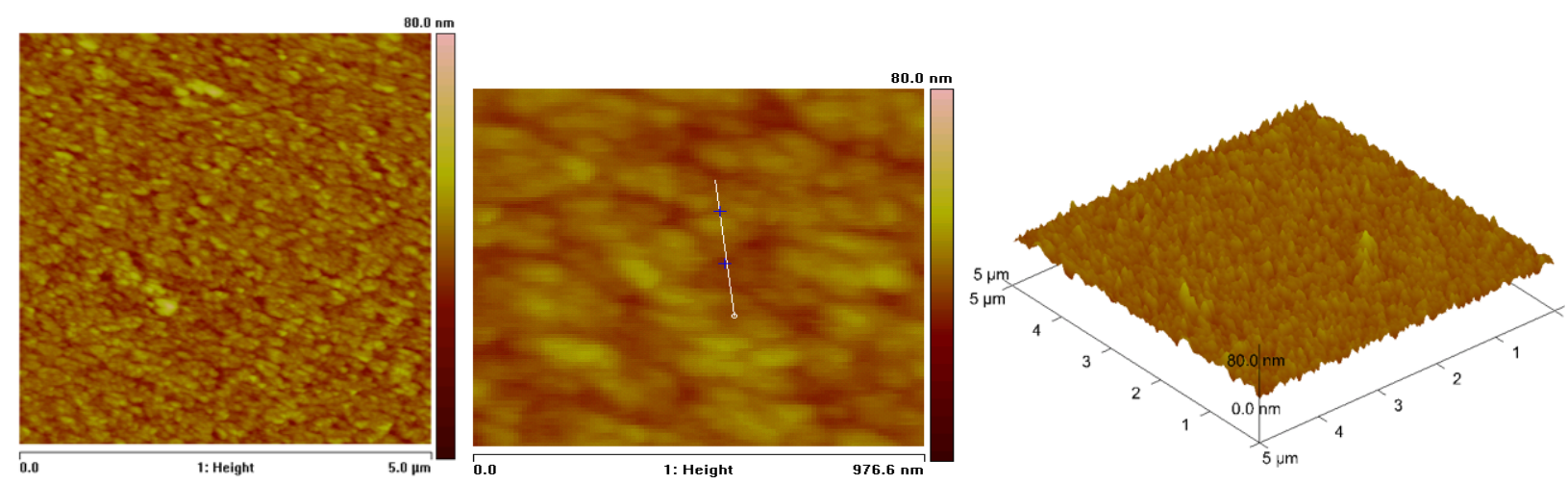

Figure 7.2 - AFM images of thin film of $39 \mathrm{~nm}$ PTCDI-C8 deposited on ITO substrate at room temperature showing $\sigma \sim$ $5.3 \pm 0.25 \mathrm{~nm}$. The ITO substrates were annealed at $500{ }^{\circ} \mathrm{C}$ in nitrogen for $10 \mathrm{~min}$ before film deposition.
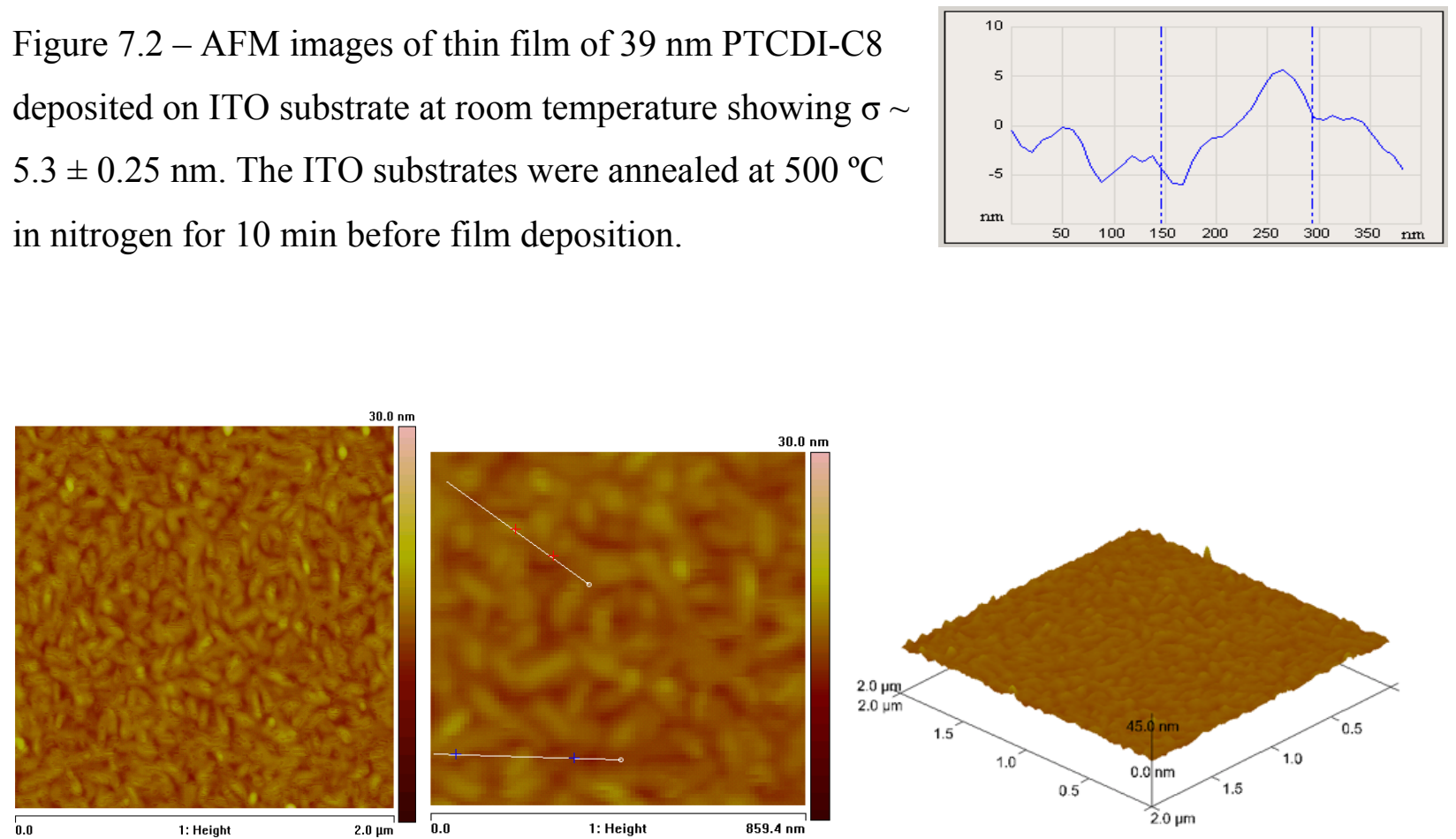

Figure 7.3 - AFM images of thin film of $27 \mathrm{~nm}$ PTCDI-C8 deposited on silicon substrate at room temperature. These films display very smooth surfaces with $\sigma \sim 1.04 \pm 0.04 \mathrm{~nm}$.

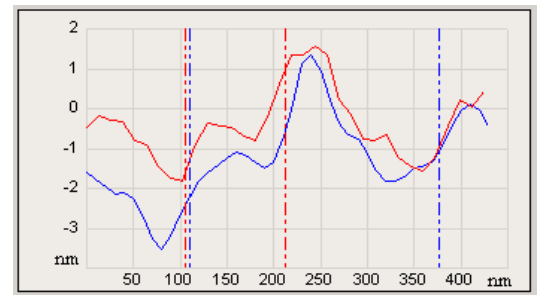


Following images (Figure 7.4) display the typical topographical AFM images of 20, 27, 33 and $45 \mathrm{~nm}$ thick PTCDI-C8 films deposited on silicon substrate at room temperature.
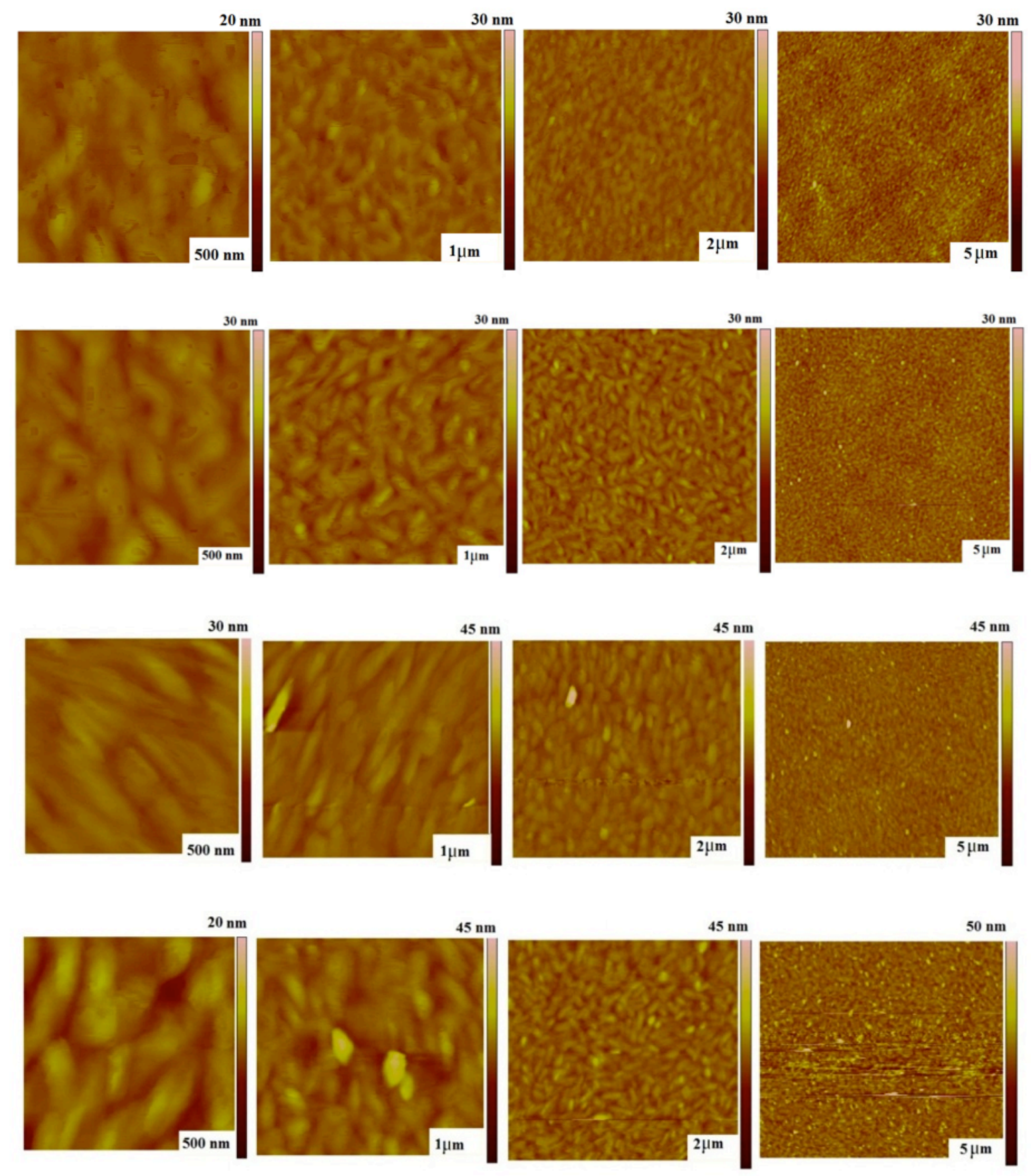

Figure 7.4 - AFM images of 20, 27, 33 and $45 \mathrm{~nm}$ thick PTCDI-C8 films deposited on silicon substrate at room temperature. 
AFM images of different thicknesses of PTCDI-C8 films display a relatively smooth surface morphology with needle-like features. These features which are randomly oriented in the film have a length of about several hundred nanometers. Planar terraces on the needles can be seen in the AFM images. The line scan of the terraces (Figure 7.3) reveals mono-molecular steps of about $2 \mathrm{~nm}$ which is close to the film periodicity determined by the X-ray data. The line scan has been performed over the area with the largest differences between the heights of the features. A summary of the roughness of the films with different thicknesses has been presented in Figure 7.5. As can be seen in this figure, the root mean square roughness increases from $0.7 \mathrm{~nm}$ to 1.7 $\mathrm{nm}$ as the film thickness increases from $20 \mathrm{~nm}$ to $45 \mathrm{~nm}$. Having a relatively smooth surface for thicker films suggests that the films display a wetting behavior on the silicon substrates [77].

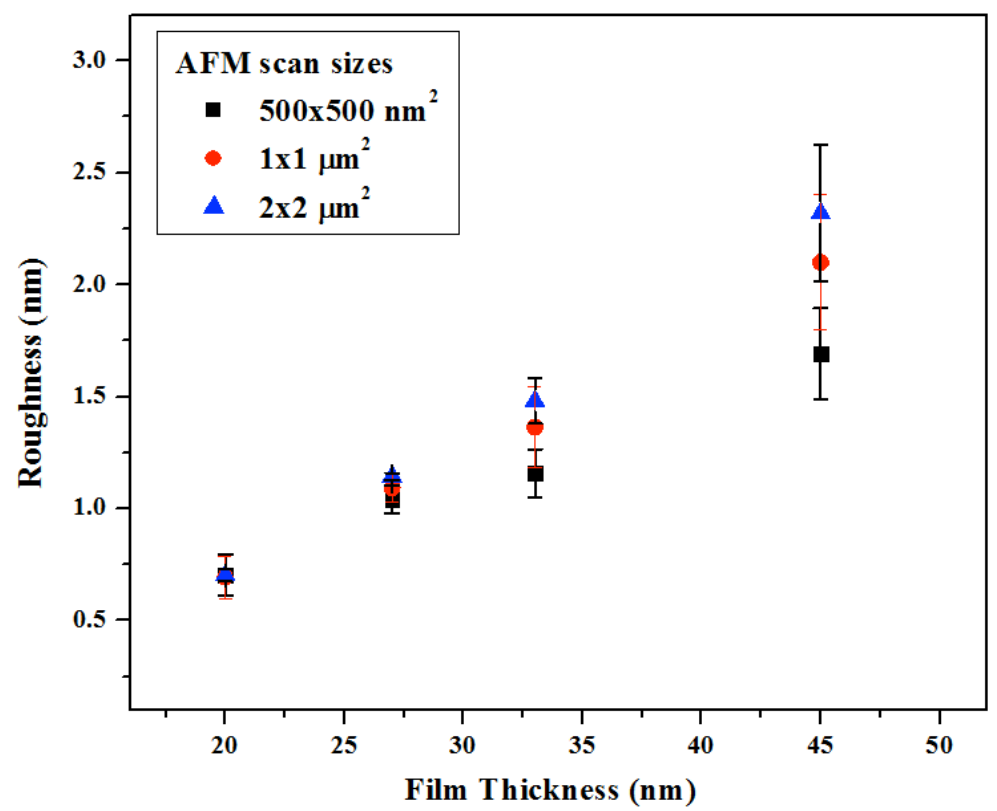

Figure 7.5 - Plot of film roughness as a function of thickness for PTCDI-C8 films deposited on silicon substrates. 


\subsection{X-RAY REFLECTIVITY (XRR)}

X-ray reflectivity data were collected for different thicknesses of PTCDI-C8 films deposited on silicon substrates with a thin layer of native oxide. The reflectivity curves presented in Figure 7.6 have been offset deliberately for better presentation. Kiessig fringes at low angles as well as the Bragg peak with un-damped Laue oscillations indicate formation of films with a highly ordered structure. The low angle Kiessig fringes are related to the total film thickness and the Laue oscillations around the Bragg peak are related to the coherently ordered film thickness. Figures 7.7 shows the measured and simulated reflectivity data for a $40 \mathrm{~nm}$ thick film of PTCDIC8. Similarity of the widths of the Kiessig fringes with the Laue oscillations around the Bragg peak suggests that PTCDI-C8 films are coherently ordered across their entire thickness.

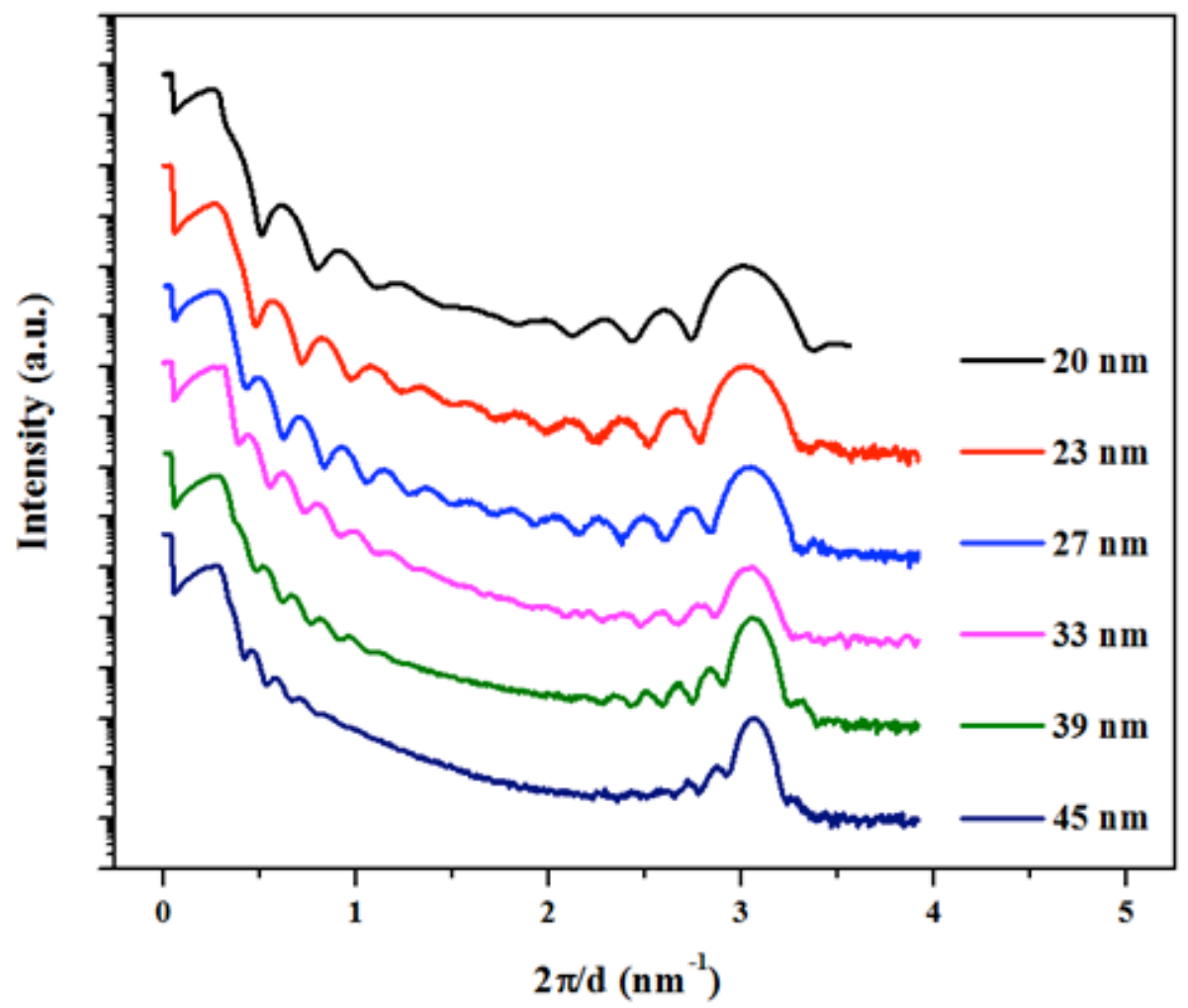

Figure 7.6 - X-ray reflectivity curves for different thicknesses of PTCDI-C8 films. 
The roughness and the thickness of the deposited films have been determined by a combination of techniques including AFM, ellipsometry and X-ray reflectivity. A summary of the roughness analysis from AFM and X-ray reflectivity has been shown in Table 2 and Figure 7.8. Thickness data from ellipsometry and reflectivity is also summarized in Table 3 and Figure 7.9. As presented in these figures, the results of characterization from different techniques are similar. Among these techniques, X-ray reflectivity is the best method to characterize the structure of the materials in a thin film since it provides detailed information about the interface and the surface roughness, film thickness, molecular ordering and film morphology.
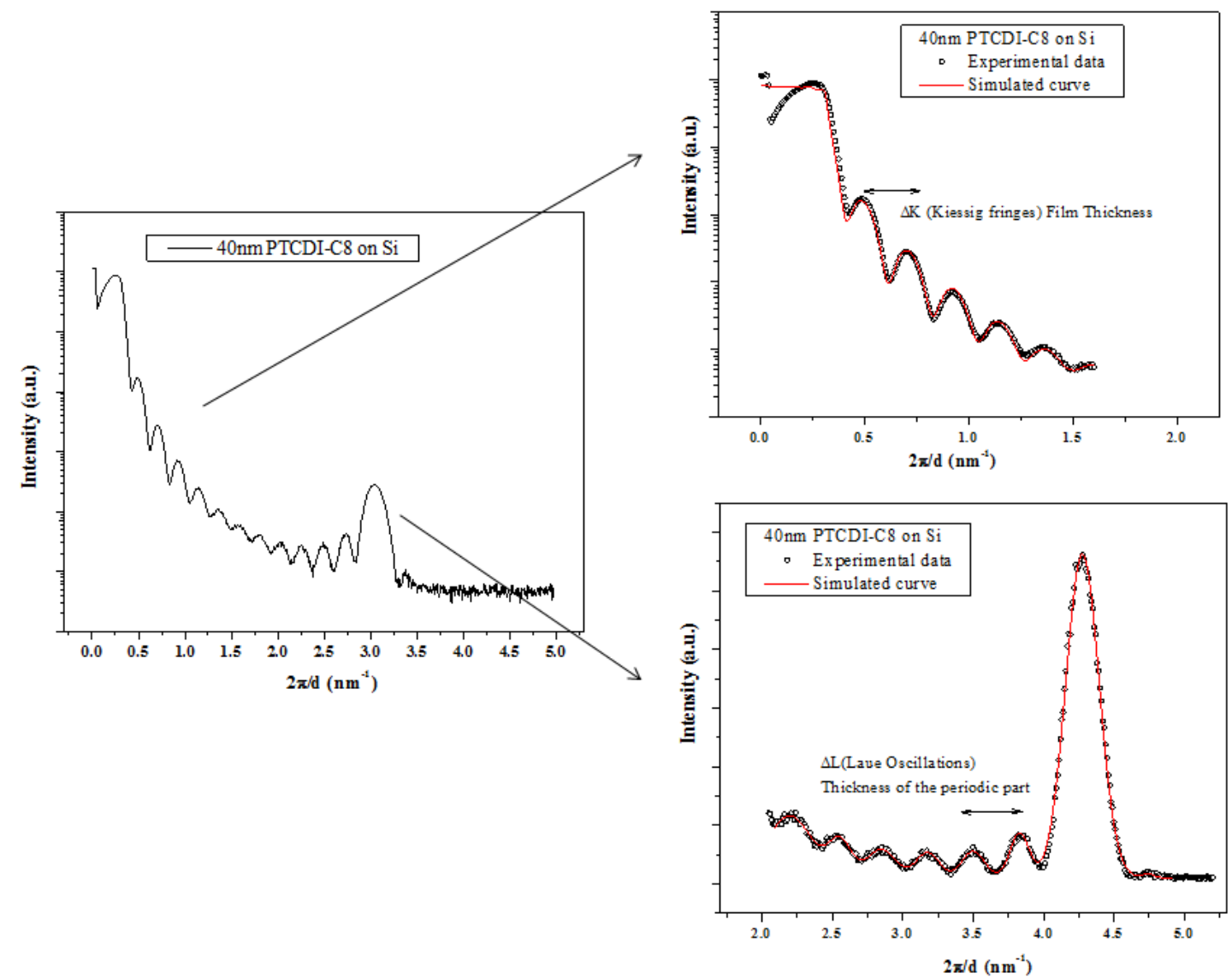

Figures 7.7 - Measured and simulated reflectivity data for PTCDI-C8 film with $27 \mathrm{~nm}$ thickness. 


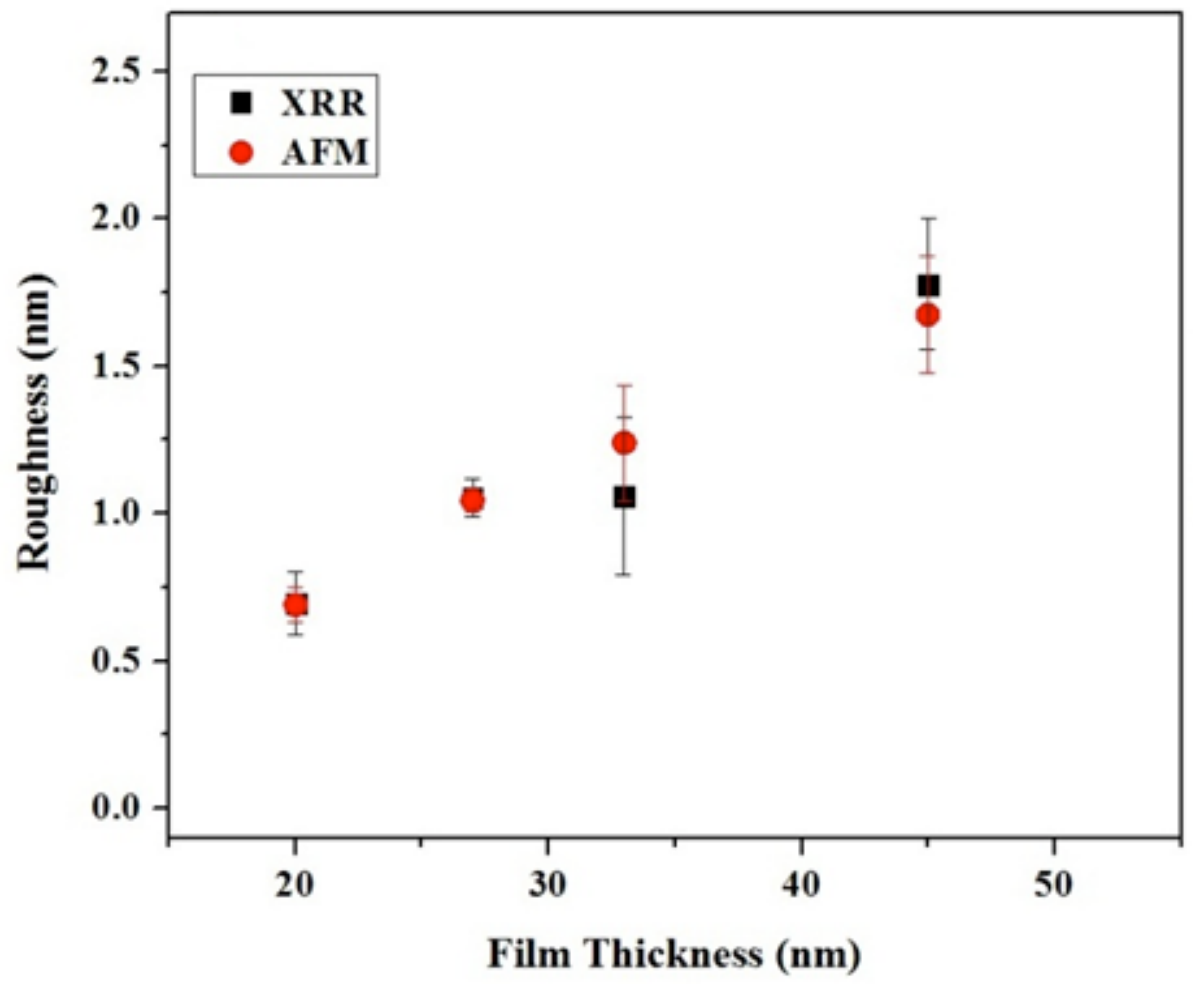

Figure 7.8 - Comparing the measured and calculated roughness from AFM and X-ray reflectivity measurements.

Table 2 - Roughness from AFM and XRR

\begin{tabular}{lllll}
\hline $\begin{array}{l}\text { Thickness (nm) } \\
\text { Crystal Monitor }\end{array}$ & $\begin{array}{l}\text { Roughness (nm) } \\
\text { XRR }\end{array}$ & Stdev & $\begin{array}{l}\text { Roughness (nm) } \\
\text { AFM }\end{array}$ & Stdev \\
\hline 20 & 0.6958 & 0.1091 & 0.69112 & 0.05888 \\
27 & 1.0539 & 0.0653 & 1.04475 & 0.03669 \\
33 & 1.0589 & 0.2672 & 1.24038 & 0.1959 \\
45 & 1.7787 & 0.2206 & 1.675 & 0.19806 \\
$\mathrm{SiO}_{2}$ & 0.72374 & 0.32708 & 0.3036 & 0.031681 \\
\hline
\end{tabular}




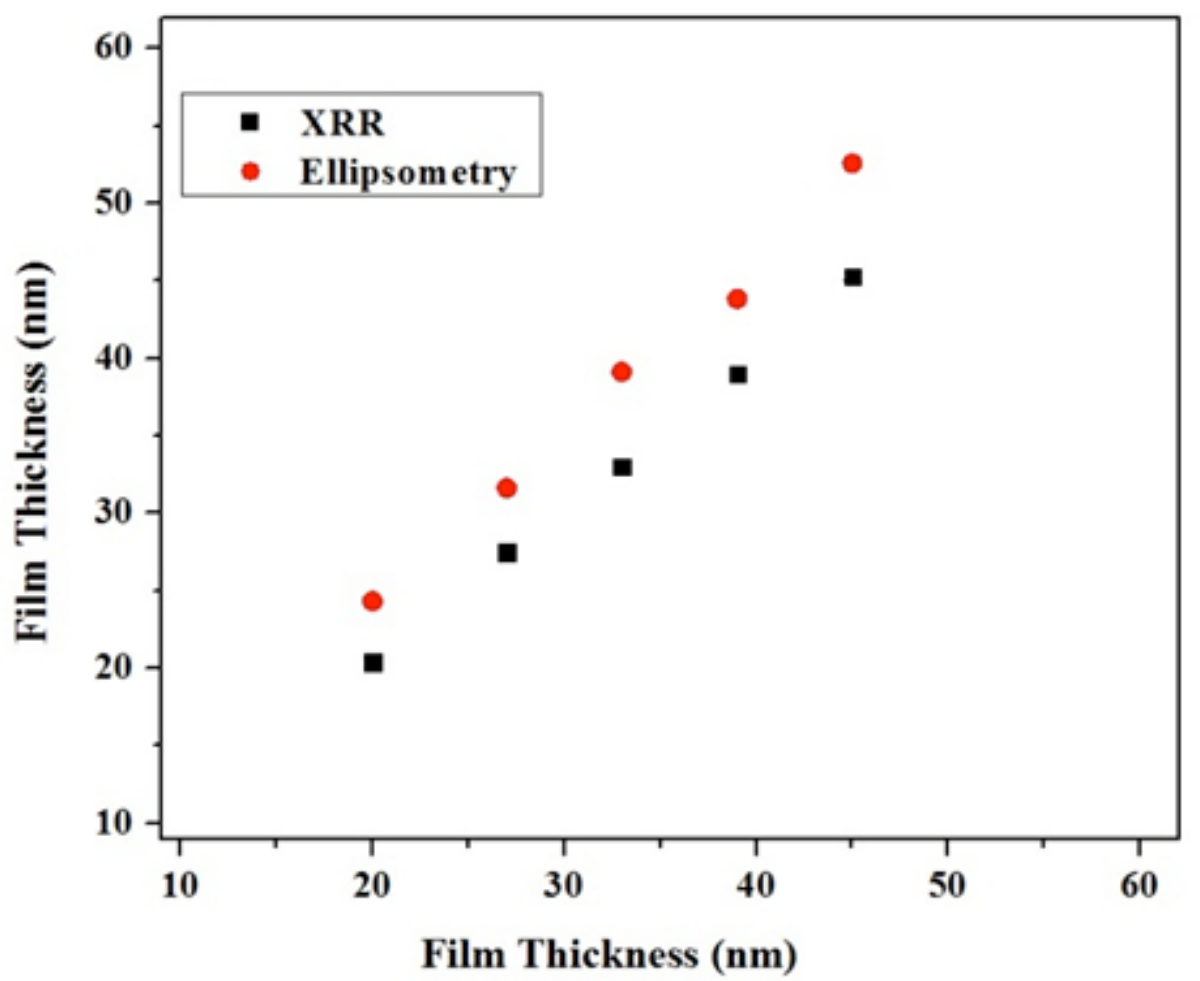

Figure 7.9 - Comparing the measured and calculated thickness from ellipsometry and X-ray reflectivity measurements.

Table 3 - Thickness from spectroscopic ellipsometry and XRR

\begin{tabular}{lllll}
\hline $\begin{array}{l}\text { Thickness (nm) } \\
\text { Crystal Monitor }\end{array}$ & $\begin{array}{l}\text { Thickness (nm) } \\
\text { XRR }\end{array}$ & Stdev & $\begin{array}{l}\text { Thickness (nm) } \\
\text { Ellipsometer }\end{array}$ & Stdev \\
\hline 20 & 20.3756 & 0.0979 & 24.326 & 0.0986 \\
27 & 27.518 & 0.1944 & 31.623 & 0.0776 \\
33 & 33.0027 & 0.1572 & 39.12 & 0.0776 \\
39 & 39.0022 & 0.2757 & 43.837 & 0.102 \\
45 & 45.2869 & 0.2637 & 52.579 & 0.132 \\
\hline
\end{tabular}


The interlayer spacing (d) can be determined from the diffraction peaks according to Bragg's equation, $\mathrm{n} \lambda=2 \mathrm{~d} \sin \theta$, where $\mathrm{n}$ is the order of the diffraction peak and $\theta$ is the scattering angle. A summary of the calculated values for PTCDI-C8 films with different thicknesses is presented in Figure 7.10. Calculated values for interlayer spacing are in good agreement with the height of the molecular terraces measured from AFM topographical images (Figures 7.2 and 7.3).

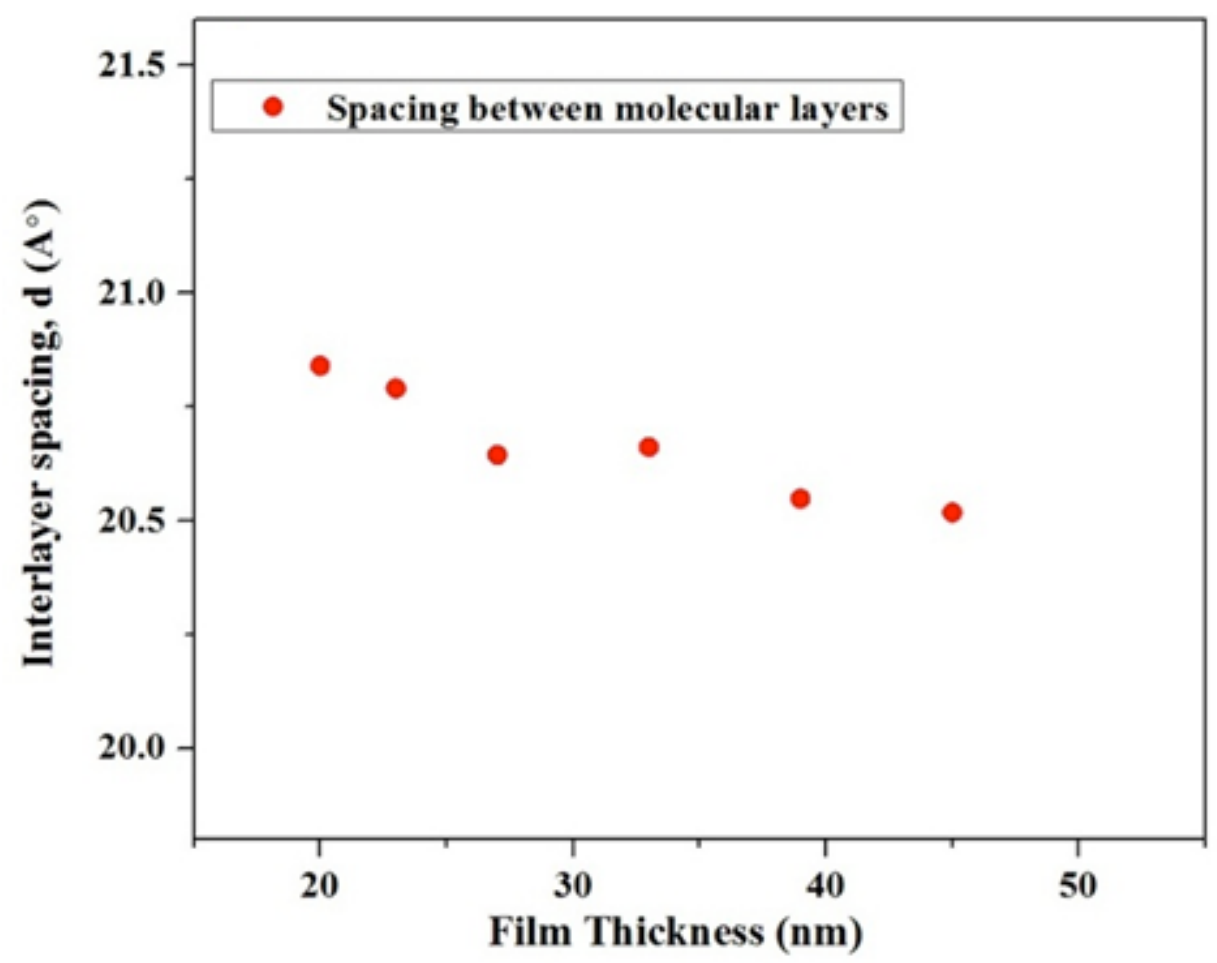

Figure 7.10 - Calculated interlayer spacing for PTCDI-C8 films with different thicknesses.

In a simplified way, considering negligible contribution from the strain, the mean size of the crystalline domains can be estimated from the inverse of the full width at half maximum (FWHM) of the Bragg peak using equation $\mathrm{D} \approx \mathrm{K} \lambda / \beta \cos \theta$, where $\mathrm{K}$ is the Scherrer constant, $\lambda$ the wavelength, $\beta$ the FWHM and $2 \theta$ is the Bragg peak angular position. The domain size 
calculated for the different thicknesses of PTCDI-C8 films are presented in Figure 7.11. As can be seen in this plot, thicker films possess larger domain size and therefore sharper peaks compared to the thinner films. The size of the crystalline domains estimated from the X-ray reflectivity measurements is in the range of 20 to $45 \mathrm{~nm}$ for different thicknesses. The fact that these values are considerably smaller than the grains size (needle-like features) observed in the AFM images indicates that the deposited films possess polycrystalline structure.

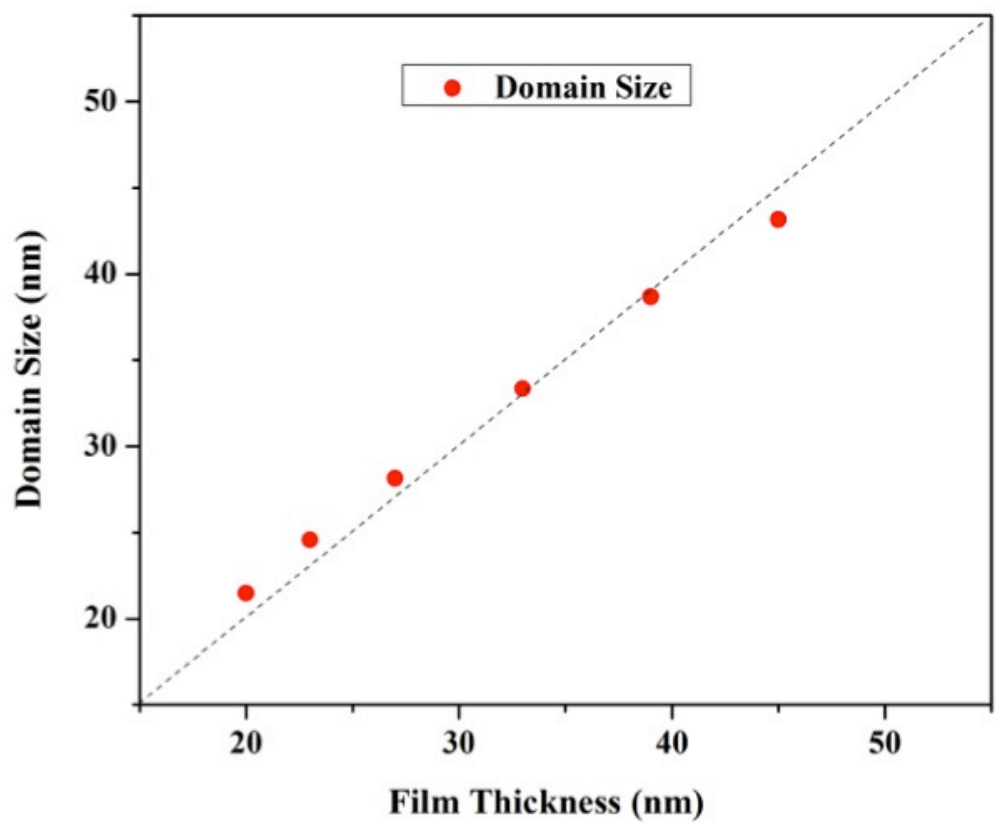

Figure 7.11 - The domain size calculated for the different thicknesses of PTCDI-C8 films. 


\subsection{ORGANIC SOLAR CELLS}

\subsubsection{Electrical characteristics}

Several solar cell devices were fabricated based on planar heterojunction (PHJ) with a sharp interface between the donor $(\mathrm{CuPc})$ and the acceptor (PTCDI-C8) layers. Each device was characterized at room temperature and under atmospheric conditions. Initially, for all of the fabricated devices, the thickness of each of the active layers (CuPc and PTCDI-C8) was fixed at $30 \mathrm{~nm}$. In order to study the effect of the variation of the thickness of the buffer layers, devices with different thicknesses of electron transport layer (ETL) and hole transport layer (HTL) were fabricated and characterized. J-V data for some of the devices with $\mathrm{MoO}_{3}$ thickness of $5 \mathrm{~nm}$ and $\mathrm{Alq}_{3}$ thickness varying from 0 to $15 \mathrm{~nm}$ are presented in Figure 8.1.

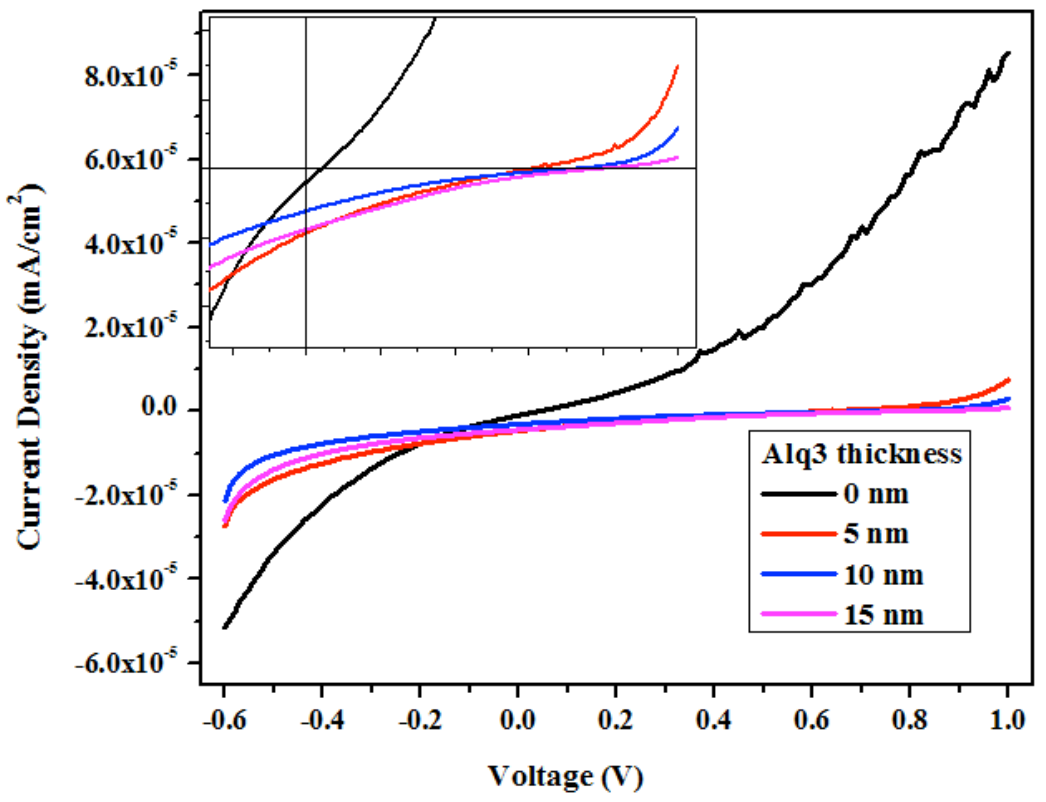

Figure $8.1-\mathrm{J}-\mathrm{V}$ data for devices with different $\mathrm{Alq}_{3}$ thicknesses ranging form 0 to $15 \mathrm{~nm}$. 
Open circuit voltage $\left(\mathrm{V}_{\mathrm{OC}}\right)$ and short circuit current $\left(\mathrm{J}_{\mathrm{SC}}\right)$ for devices with different ETL and HTL thicknesses are presented in Figures 8.2 and 8.3. As can be seen in these plots, $V_{O C}$ increases with an increase in ETL or HTL. The maximum $\mathrm{J}_{\mathrm{SC}}$ for devices with various HTL was observed at $10 \mathrm{~nm}$ thickness of $\mathrm{MoO}_{3}$ and for devices with various EHL at $5 \mathrm{~nm}$ thickness of $\mathrm{Alq}_{3}$. Experimental results for the studied solar cell structures in this work prove the effect of the thickness variation of the buffer layers on the output characteristics of the devices.

In order to study the effect of thickness variation of the active layers, devices with different thicknesses of $\mathrm{CuPc}$ and PTCDI-C8 were fabricated and characterized. J-V results and a summary of the $\mathrm{V}_{\mathrm{OC}}$ and $\mathrm{J}_{\mathrm{SC}}$ for some of these devices are presented in Figure 8.4 and Figure 8.5. Devices with total active layer thickness of $40 \mathrm{~nm}$ show the best electrical characteristics for the fabricated devices.
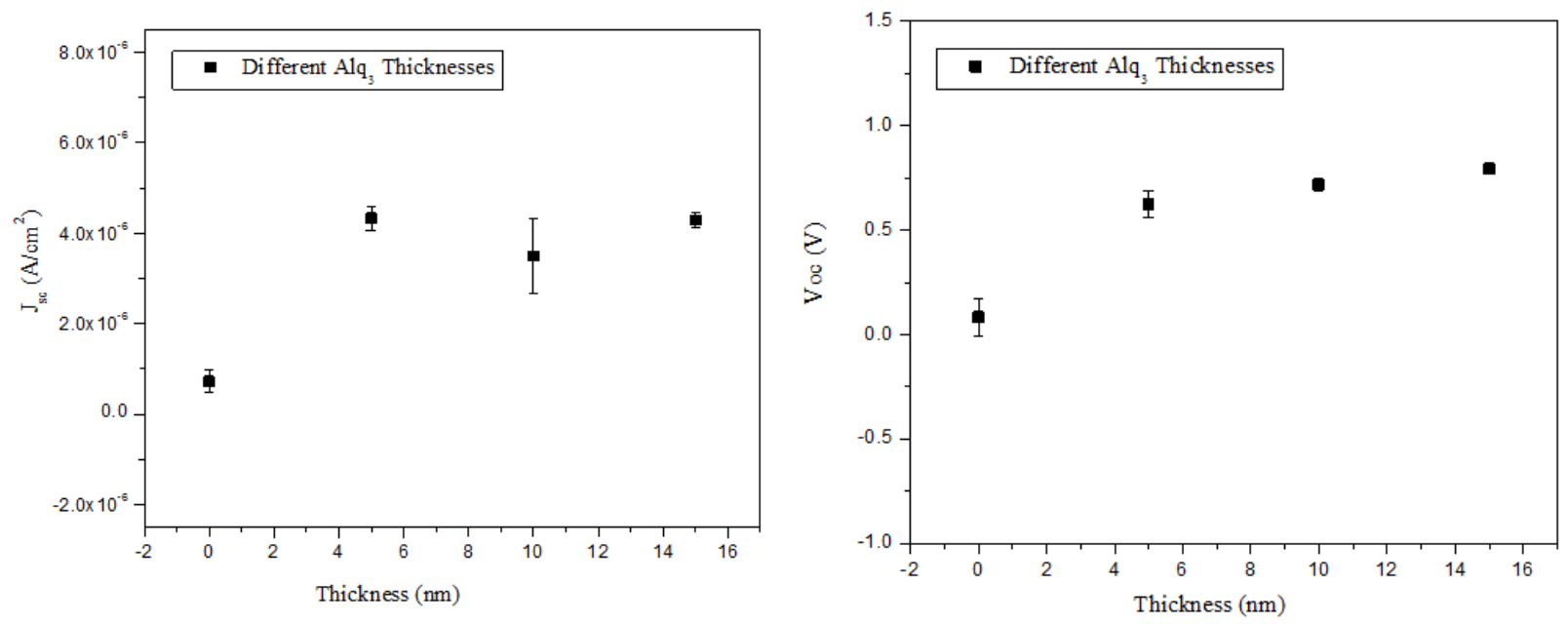

Figure 8.2 - Open circuit voltage $\left(\mathrm{V}_{\mathrm{OC}}\right)$ and short circuit current $\left(\mathrm{J}_{\mathrm{SC}}\right)$ for devices with different ETL thickness in the following structure: $\mathrm{MoO}_{3} / \mathrm{CuPc} / \mathrm{PTCDI} / \mathrm{Alq}_{3} / \mathrm{Al}(5 \mathrm{~nm} / 30 \mathrm{~nm} / 30 \mathrm{~nm} / \mathrm{X}$ $\mathrm{nm} / 75 \mathrm{~nm})$. 

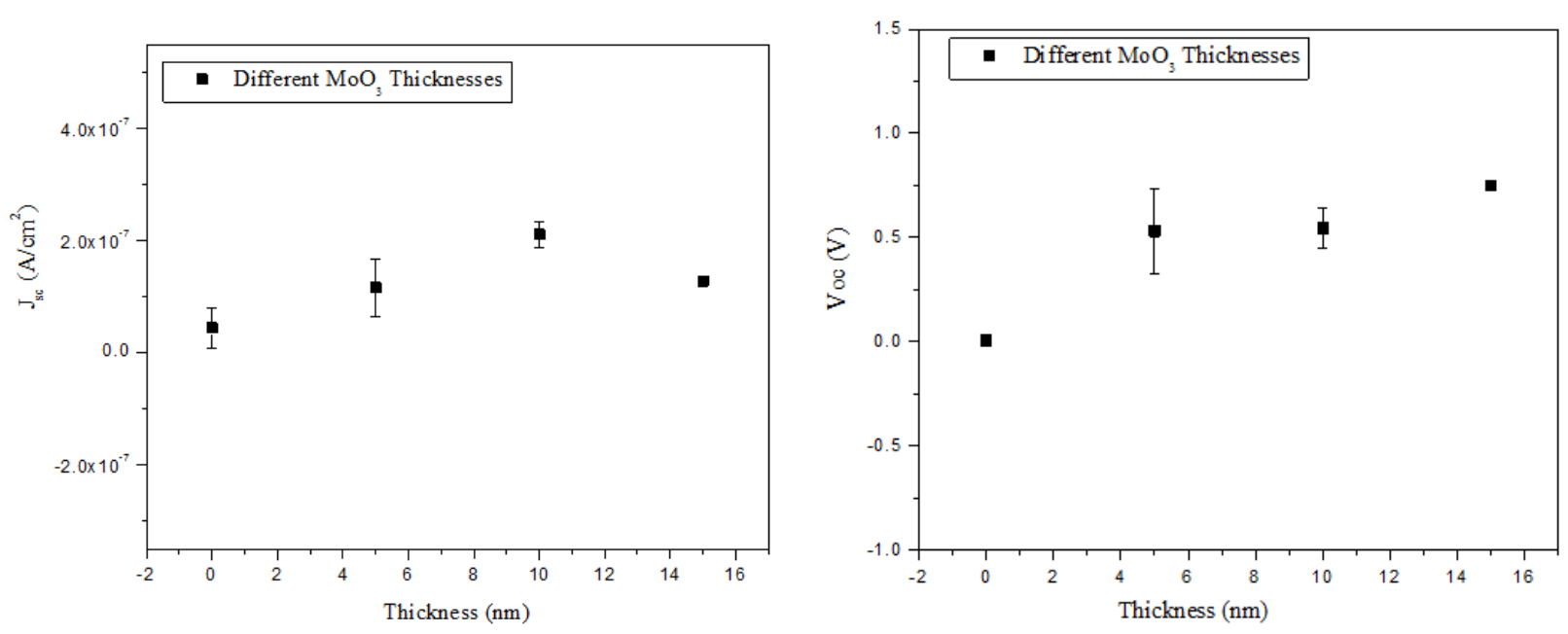

Figure 8.3 - Open circuit voltage $\left(\mathrm{V}_{\mathrm{OC}}\right)$ and short circuit current $\left(\mathrm{J}_{\mathrm{SC}}\right)$ for devices with different HTL thickness in the following structure: $\mathrm{MoO}_{3} / \mathrm{CuPc} / \mathrm{PTCDI} / \mathrm{Alq}_{3} / \mathrm{Al}(\mathrm{X} \mathrm{nm} / 30 \mathrm{~nm} / 30 \mathrm{~nm} / 7.5$ $\mathrm{nm} / 75 \mathrm{~nm}$ )
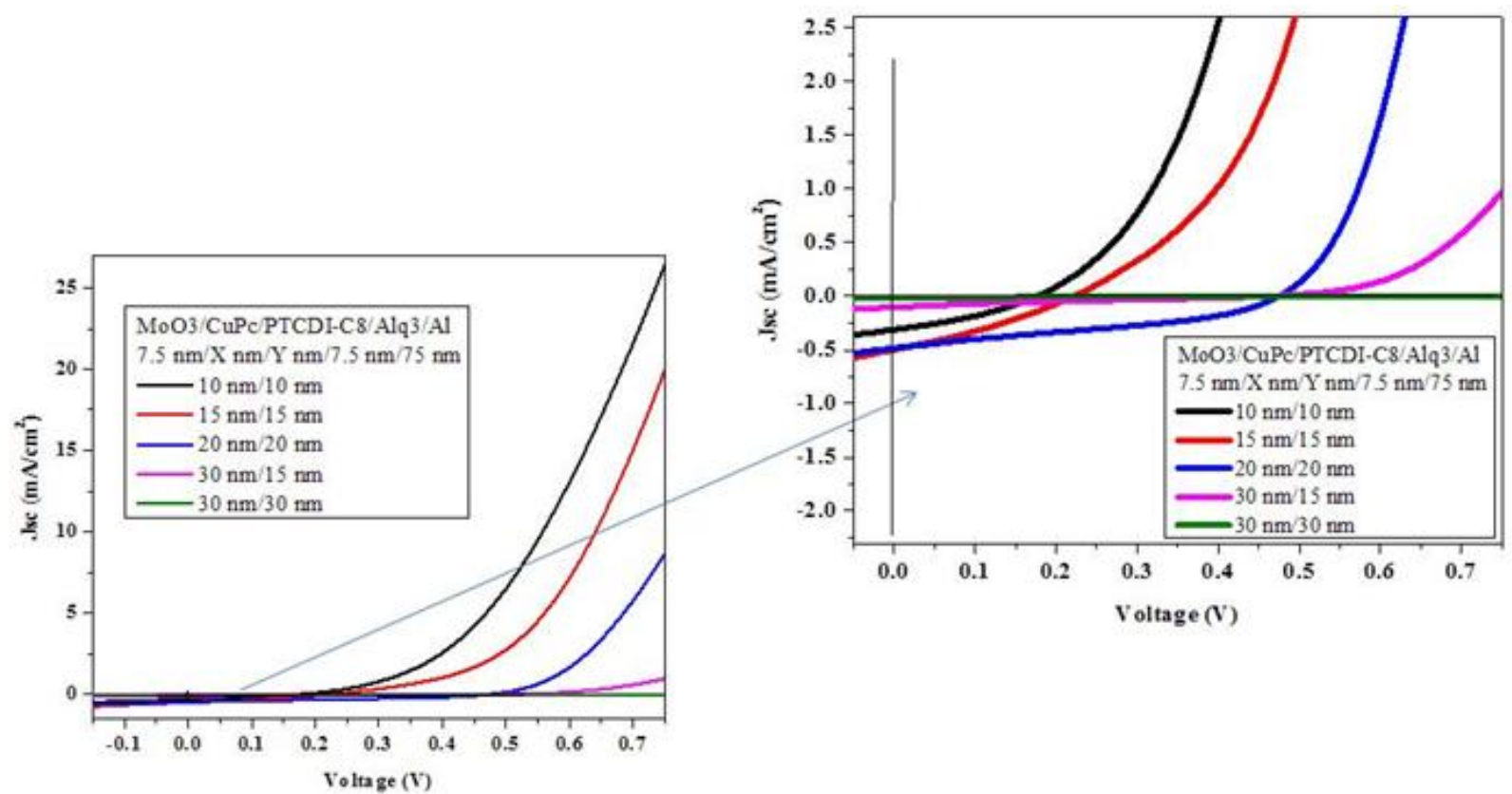

Figure $8.4-\mathrm{J}-\mathrm{V}$ data for devices with different active layers thicknesses in the following structure: $\mathrm{MoO}_{3} / \mathrm{CuPc} / \mathrm{PTCDI}-\mathrm{C} 8 / \mathrm{Alq}_{3} / \mathrm{Al}(7.5 \mathrm{~nm} / \mathrm{X} \mathrm{nm} / \mathrm{Y} \mathrm{nm} / 7.5 \mathrm{~nm} / 75 \mathrm{~nm})$ 

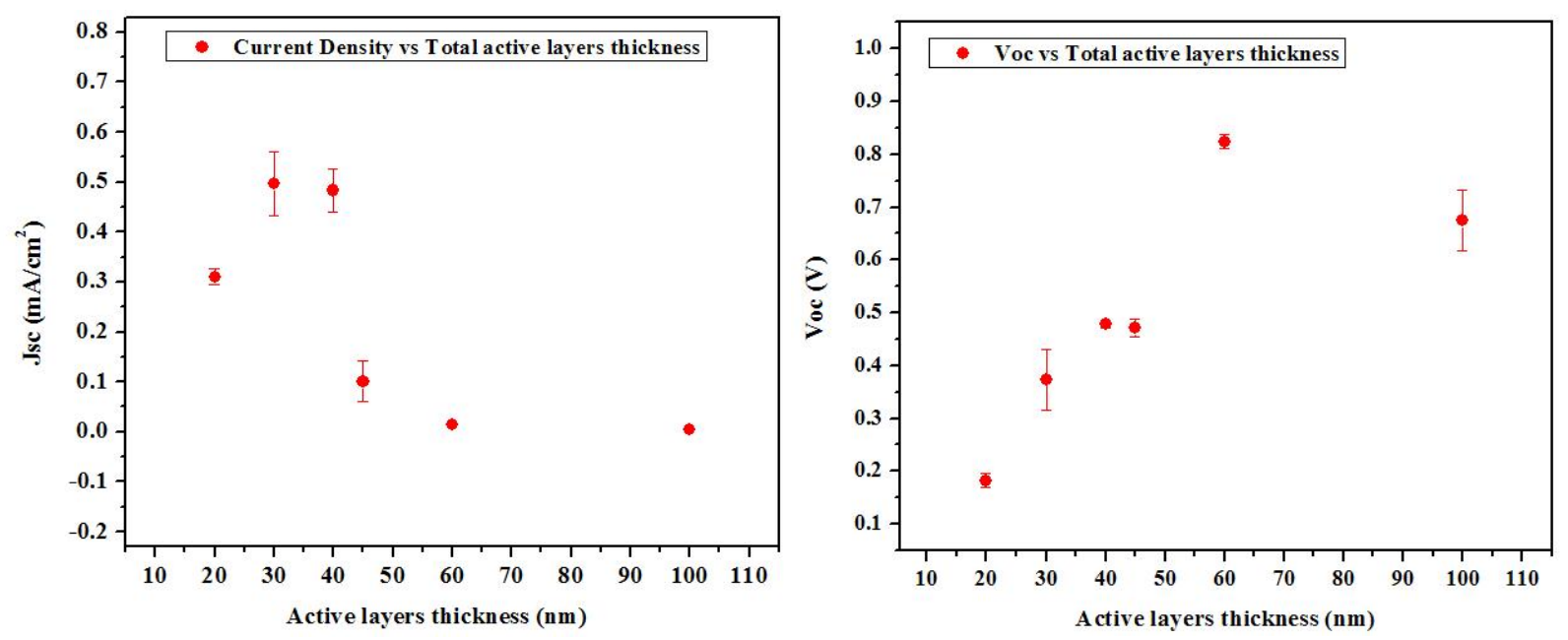

Figure 8.5 - Open circuit voltage $\left(\mathrm{V}_{\mathrm{OC}}\right)$ and short circuit current $\left(\mathrm{J}_{\mathrm{SC}}\right)$ for devices with different active layers thickness in the following structure: $\mathrm{MoO}_{3} / \mathrm{CuPc} / \mathrm{PTCDI} / \mathrm{Alq}_{3} / \mathrm{Al}(7.5 \mathrm{~nm} / \mathrm{X} \mathrm{nm} / \mathrm{Y}$ $\mathrm{nm} / 7.5 \mathrm{~nm} / 75 \mathrm{~nm})$

Several devices with total active layers thickness of $40 \mathrm{~nm}$ and different thicknesses for $\mathrm{Alq}_{3}$ layer have been fabricated and characterized. The effects of the thickness variation of the $\mathrm{Alq}_{3}$ layer on the electrical characteristics of these devices have been studied. Under illumination, excitons (bonded electron/hole) are generated in the active layer materials and dissociated into electrons and holes at the donor/acceptor interface. The difference between the work function of the electrodes results in a built-in electric field which drives the generated charge carriers toward the electrodes, where these carriers will be collected respectively [78]. The ability to collect these charge carriers in an efficient manner is of significant importance. Therefore, the contact between the organic layer and the electrode is one of the most critical interfaces that determine the efficiency of the OSCs. Inserting additional layers between organic materials and electrodes is one of the most efficient approaches to modify their interface [43, 79]. Although this interface modification enhances the optical and electrical characteristics of the 
OSCs, it may result in performance degradation of the cells. One of the most commonly observed effects is the S-shape I-Vs that reduce the fill factor (FF) and power conversion efficiency of the cells significantly [80]. S-shape effect has been observed in different structures of OSCs such as small molecule, polymer, hybrid and tandem structures [80-82]. Several different possible explanations for this phenomenon have been proposed which include strong interface dipoles, charge accumulation, injection and extraction barriers between the hole/electron transport layers and electrodes [80 - 82]. In this section, the effect of the electron transport layer thickness on the electrical properties of the OSCs has been studied.

Figure 8.6 shows the J-V curves of the OSCs with different Alq3 thicknesses. The short circuit current density $\left(\mathrm{J}_{\mathrm{SC}}\right)$ for devices without Alq3 and with $5 \mathrm{~nm}$ Alq3 is approximately 3 and $5 \mu \mathrm{A} / \mathrm{cm} 2$, respectively. Increasing Alq3 thickness from $10 \mathrm{~nm}$ to $30 \mathrm{~nm}$ results in an increase of

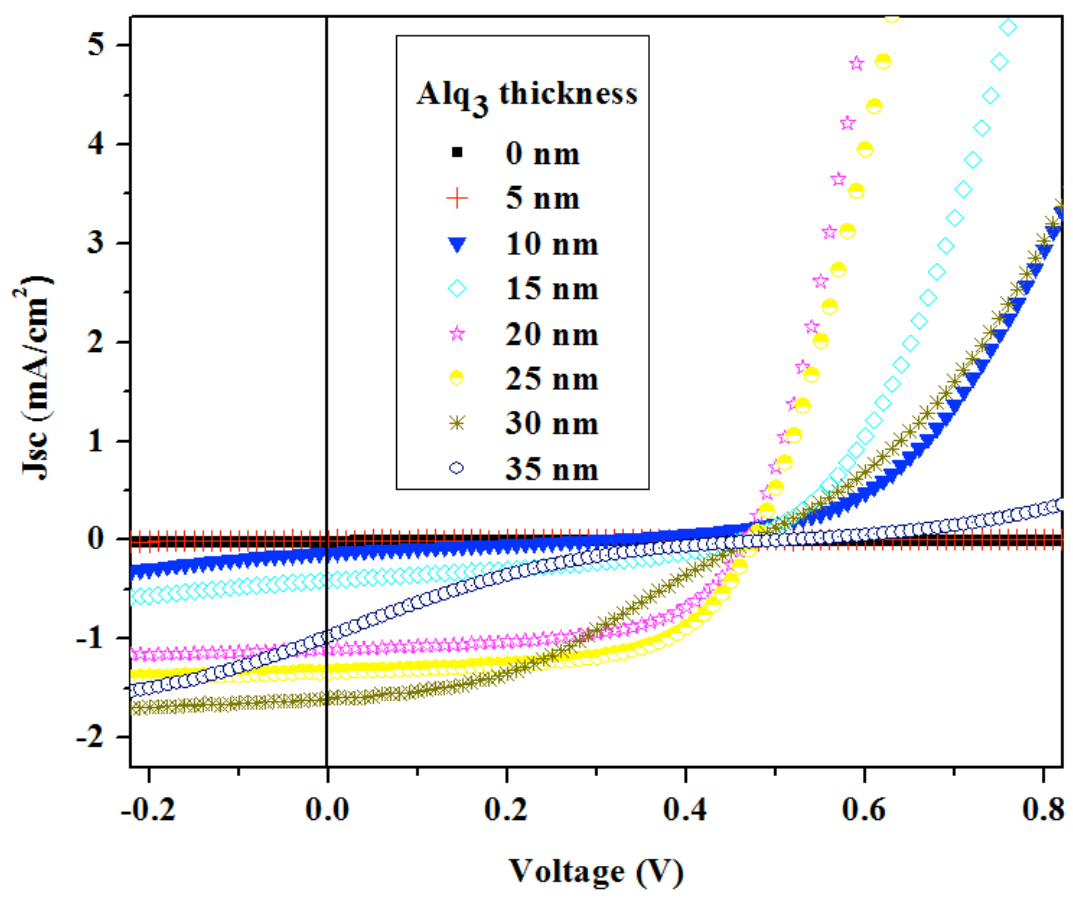

Figure 8.6 - Current density of different devices as a function of applied bias under light illumination for various $\mathrm{Alq}_{3}$ thicknesses. 
the $\mathrm{J}_{\mathrm{SC}}$ from 0.1 to $1.6 \mathrm{~mA} / \mathrm{cm} 2$, which indicates an improvement by a factor of 16 . The $\mathrm{FF}$ also improves from $24.3 \%$ to $59.5 \%$ yielding an efficiency of $0.38 \%$ when increasing the $\mathrm{Alq}_{3}$ thickness from 10 to $25 \mathrm{~nm}$. In spite of the dramatic change observed in $\mathrm{J}_{\mathrm{SC}}$ and $\mathrm{FF}$, the open circuit voltage $\left(\mathrm{V}_{\mathrm{OC}}\right)$ remains almost constant at about $0.5 \mathrm{~V}$ for devices with $\mathrm{Alq}_{3}$ thicknesses larger than $15 \mathrm{~nm}$. As can be seen in Figure 8.6, for devices with 15, 20 and $25 \mathrm{~nm}$ of $\mathrm{Alq}_{3}$, the normal exponential $\mathrm{J}-\mathrm{V}$ curves were observed. While increasing the thickness of the $\mathrm{Alq}_{3}$ layer, an S-shape characteristic appears, resulting in a significant drop in the FF to $14.8 \%$ for the devices with $35 \mathrm{~nm}$ thickness of $\mathrm{Alq}_{3}$. A summary of the performance parameters for different devices can be seen in Figure 8.7. It has been shown that during cathode deposition, hot metal atoms can diffuse into the organic layer and modify the electrical properties of the organic and the contact layers [80]. High leakage current and pinning of the Fermi level due to introducing interfacial dipoles and defect states are some of the typical observed effects that result in the device performance degradation [78]. Therefore, inserting a buffer layer between the organic and electrode layers can suppress the diffusion and reaction of the metal ions significantly. The buffer layer should be thick enough to have a complete coverage over the organic layer against the metal deposition damages. For fabricated devices in this work, the buffer layer thickness at which an increase in the $J_{\mathrm{SC}}$ can be seen was found to be approximately $10 \mathrm{~nm}$.

In organic solar cells, the organic layers are sandwiched between a transparent and a reflective electrode. Under illumination a standing wave with zero optical field intensity at the organic/electrode interfaces is generated inside the active layer. Depending on the refractive indices and the thicknesses of the organic layers, maximum intensity is formed inside the organic 

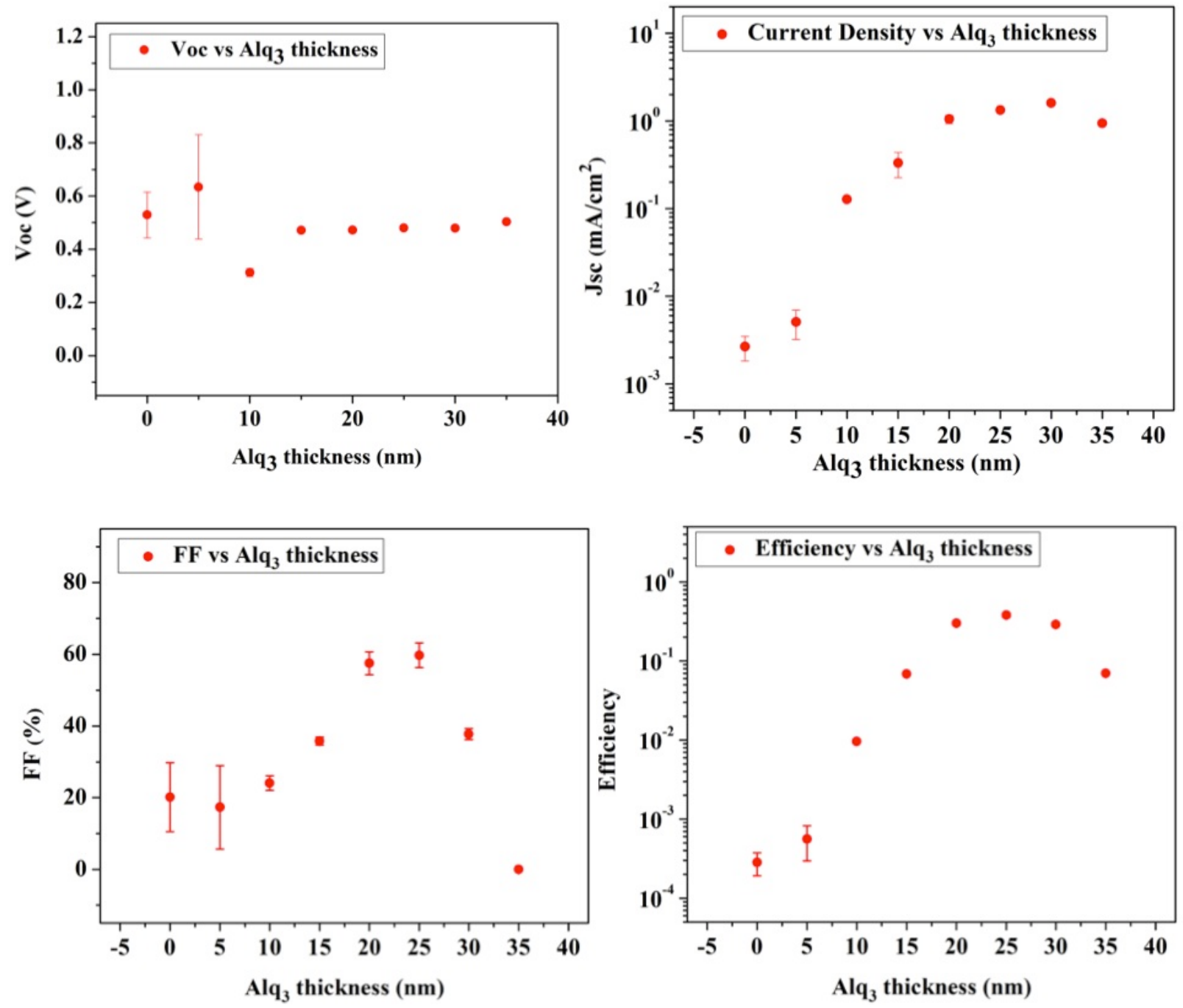

Figure 8.7 - Open circuit voltage $\left(\mathrm{V}_{\mathrm{OC}}\right)$, short circuit current $\left(\mathrm{J}_{\mathrm{SC}}\right)$, fill factor $(\mathrm{FF})$, efficiency $(\eta)$ and Pmaxfor devices with different $\mathrm{Alq}_{3}$ thickness.

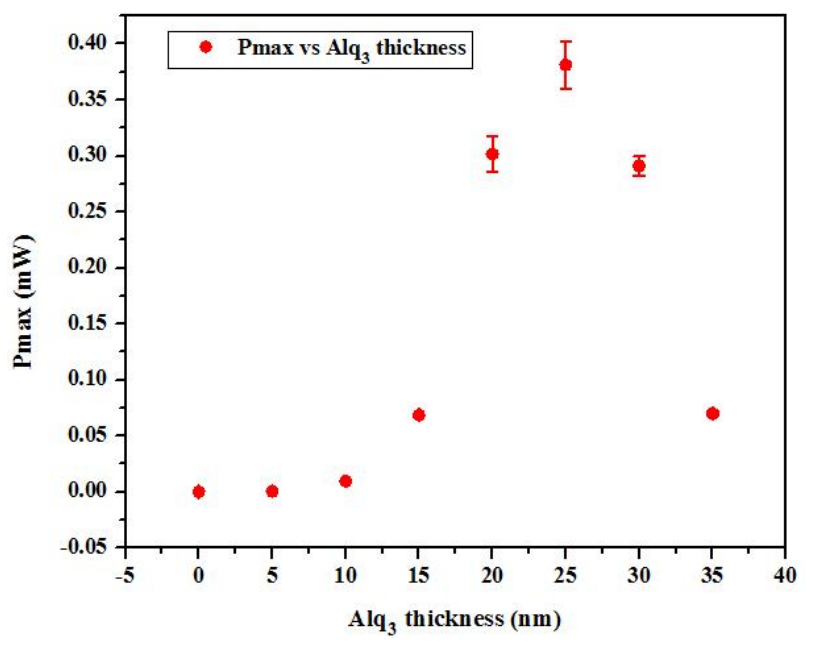


layer at a certain distance from the refractive electrode. Inserting an optically transparent layer can readjust the field distribution and shift the maximum to the donor/acceptor interface, which in turn can lead to a gain in $\mathrm{J}_{\mathrm{SC}}$ [83]. Thickness of the active layer for organic photovoltaic cells is limited by the short diffusion length and low mobility of the carriers. Introducing a buffer layer between the organic layer and each of the electrode layers can be one of the most effective ways to increase the $\mathrm{J}_{\mathrm{SC}}$ for thin film OSCs. As can be seen in Figure 8.6, increasing the $\mathrm{Alq}_{3}$ thickness from 10 to $30 \mathrm{~nm}$ results in an increase of $J_{\mathrm{SC}}$ from 0.13 tol.6 $\left(\mathrm{mA} / \mathrm{cm}^{2}\right)$ for the fabricated devices. As $\mathrm{Alq}_{3}$ thickness increases, the exponential diode curve degrades and an Sshape kink appears in the J-V plots. This effect results in a major drop in FF and has been observed for the devices with 30 and $35 \mathrm{~nm} \mathrm{Alq}_{3}$ thicknesses. As can been seen in Figure 8.8, the S-shape kink was not observed in the dark currents which imply that it is an effect of the illumination and photogenerated carriers. When the buffer layer is thin enough, photogenerated charge carriers move toward the cathode via the metal-induced defect states under the LUMU level of $\mathrm{Alq}_{3}$ as illustrated in Figure 8.9. These charges will be extracted by the cathode, resulting in an exponential curve. On the other hand, in devices with thicker buffer layers, charge extraction will be hindered due to the resistance of the bulk $\mathrm{Alq}_{3}$ layer, resulting in charge accumulation and recombination at the interface between the active and the buffer layers and degradation in the performance of the device [84]. 


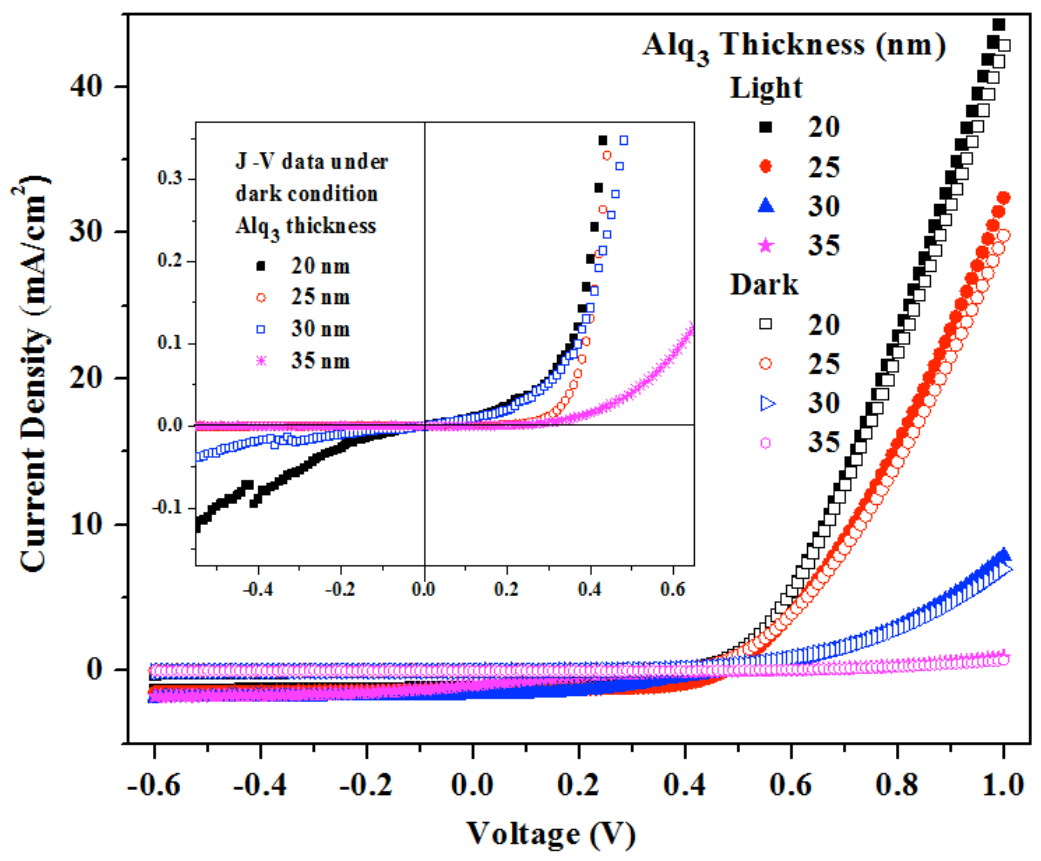

Figure $8.8-\mathrm{J}-\mathrm{V}$ curves of devices with 20,25, 30 and $35 \mathrm{~nm} \mathrm{Alq}_{3}$ thicknesses in dark and under illumination.

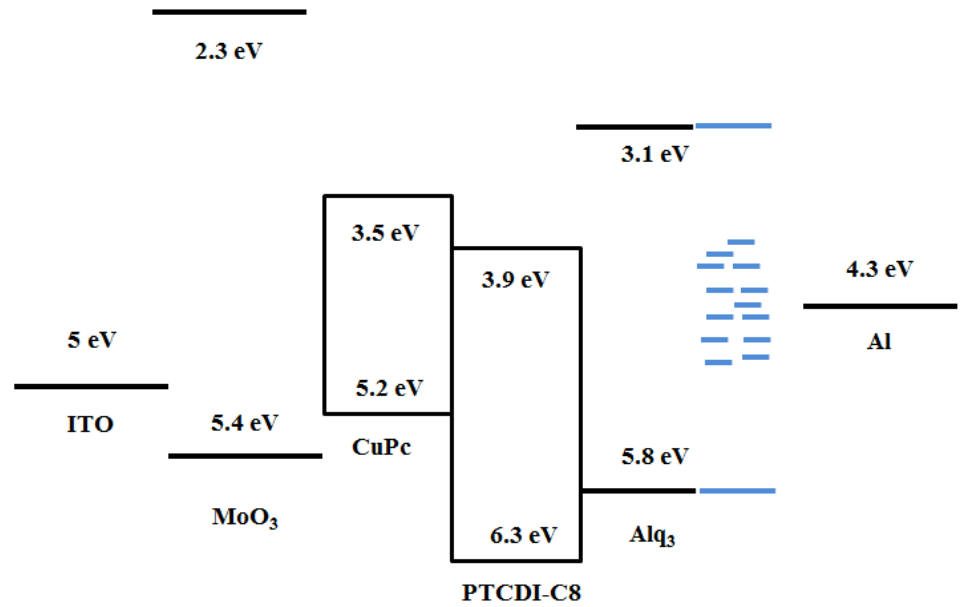

Figure 8.9 - Schematic energy diagram of $\mathrm{CuPc} / \mathrm{PTCDI}-\mathrm{C} 8$ bilayer solar cells showing the effect of metal-induces defect states distribution throughout the $\mathrm{Alq}_{3}$ buffer layer [80, 8587]. 


\subsubsection{Optical characteristics}

Typically an organic solar cell consists of the organic layers sandwiched between a transparent and a highly reflective electrode. There are different parameters that affect the power conversion efficiency of a solar cell such as light absorption and charge carrier extraction. Among these parameters, light absorption strongly depends on the optical properties of the layers and the device structure. The main goal of the device design is to optimize the light absorption which can be done, for instance, by light trapping utilizing folded solar cells. One other effective way to optimize the light absorption and eventually power conversion efficiency of organic solar cells is to fabricate devices with tuned layers. This layered structure can result in minimizing the reflectivity effect and bring most of the absorbed light to the interface between the two active layers where the charge separation occurs. In order to fabricate such a device, we need to study the effect of the optical properties of each layer. In this work, we have studied the effect of the

thickness variation of different layers on the light spectrum reaching the active layers by simulating the reflectivity of each layer. Our approach is to use the transfer matrix formalism which is a combination of a matching and a propagation matrix relating the fields across different interfaces. Using this method, the total reflectance can be modeled as follows [88, 89],

$$
\begin{aligned}
& {\left[\begin{array}{l}
B \\
C
\end{array}\right]=\prod_{r=1}^{q}\left[\begin{array}{cc}
\cos \left(d_{r}\right) & \frac{i \sin \left(d_{r}\right)}{n_{r}} \\
i n_{r} \sin \left(d_{r}\right) & \cos \left(d_{r}\right)
\end{array}\right]\left[\begin{array}{c}
1 \\
n_{s u b s}
\end{array}\right]} \\
& R=\left(\frac{1-Y}{1+Y}\right) \overline{\left(\frac{1-Y}{1+Y}\right)} \\
& d_{r}=\frac{\lambda}{4 n_{r}}, \quad Y=\frac{C}{B}
\end{aligned}
$$


where $\mathrm{d}$ is the quarterwave thickness,

$\mathrm{n}_{\mathrm{r}}$ is the refractive index of the layer $\mathrm{r}$,

$\mathrm{Y}$ is the optical admittance and $\mathrm{R}$ is the magnitude of the reflectance. This method uses the film thicknesses and refractive indices of each layer to calculate the optical impedance and the total reflectivity of the structure. The required information, thickness

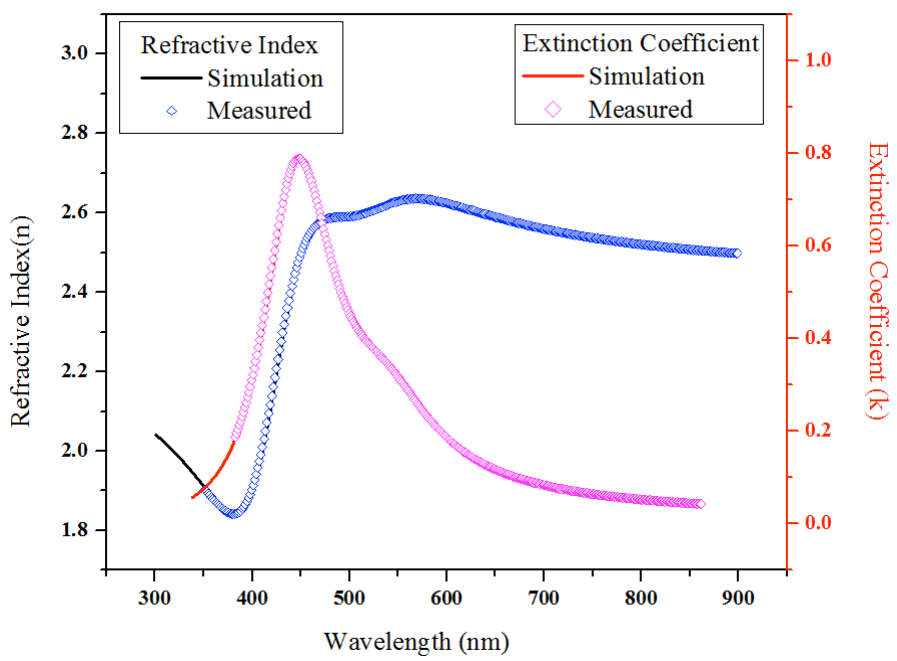

Figure 8.10 - Simulated and measured refractive indices and extinction coefficients for $\mathrm{MoO}_{3}$. and refractive index of each layer, were obtained by performing the ellipsometry technique. Ellipsometry data for organic layers and $\mathrm{MoO}_{3}$ film were fitted using Lorentz model. The MATLAB code used to calculate the refractive indices and extinction coefficients as well as the simulated data are presented in Appendix A. Figure 8.10 shows the simulated and measured data for $\mathrm{MoO}_{3}$.

In order to calculate reflectivity, a MATLAB code using the transfer matrix method was prepared (Appendix B). The effect of the thickness variation of different layers on the total reflectivity has been simulated. Simulated reflectivity for different ITO layer thicknesses is presented in Figure 8.11 (a). Figure 8.11 (b) shows the reflectivity for structures with $170 \mathrm{~nm}$ ITO and different $\mathrm{MoO}_{3}$ thicknesses from 0 to $40 \mathrm{~nm}$. Figure 8.11 (c) shows the reflectivity for the structures with the same thicknesses for ITO $(170 \mathrm{~nm})$ and $\mathrm{MoO}_{3}(20 \mathrm{~nm})$ and different $\mathrm{CuPc}$ thicknesses from 0 to $70 \mathrm{~nm}$. As can be seen in these figures, increasing the thickness of the $\mathrm{MoO}_{3}$ layer shifts the reflectivity peaks to higher wavelengths and also increases the total reflectance. For structures with different CuPc thicknesses, increasing the thickness results in an 
increase of the total reflectivity to about $30 \%$. Figures 8.11 (d) and 8.12 show the calculated reflectivity for different thicknesses of PTCDI-C8 and $\mathrm{Alq}_{3}$, respectively. As presented in these figures, thickness variation for each layer affects the total reflectivity of all layers. In order to verify the simulation results, samples were prepared and their reflectivities were measured and
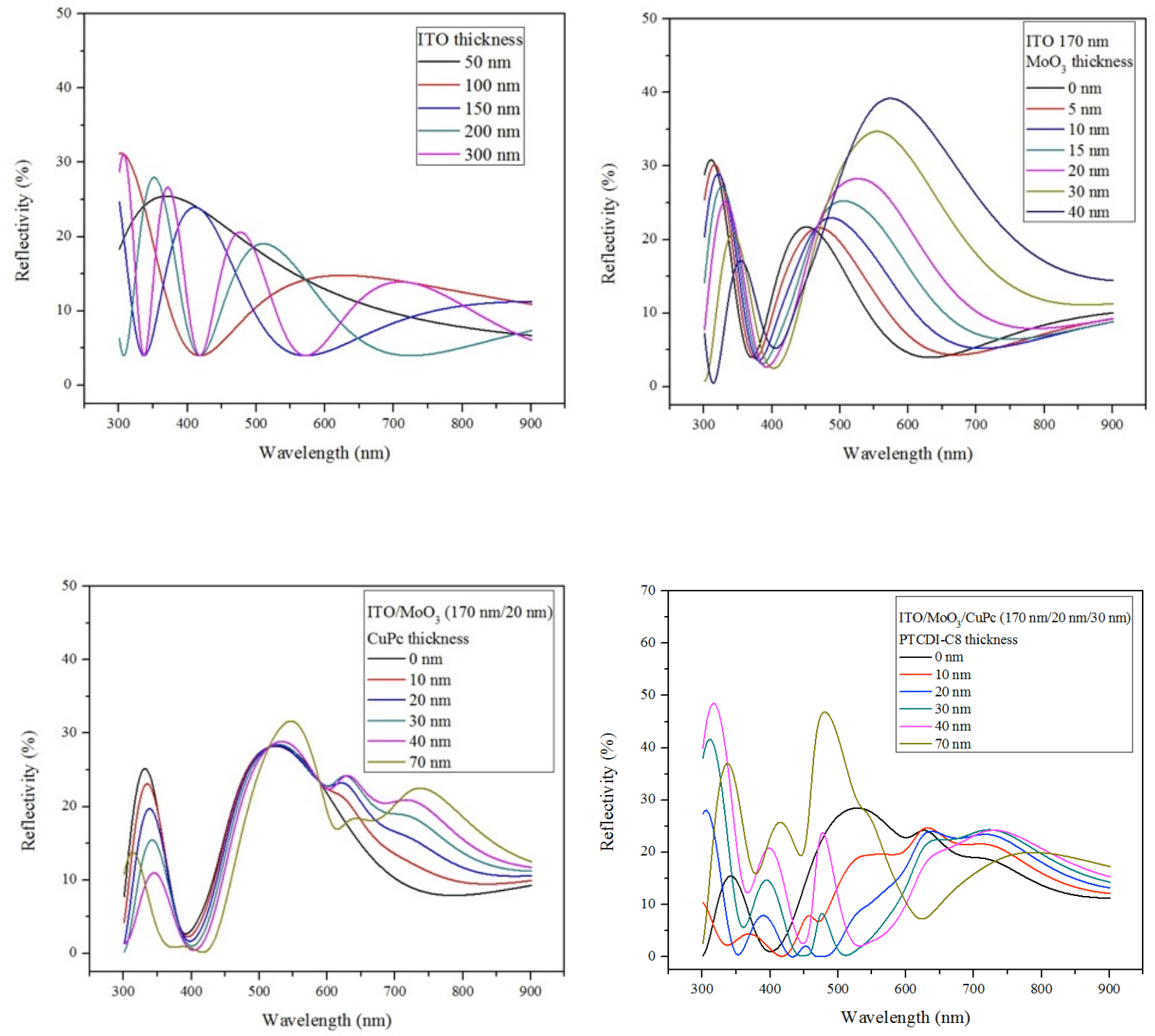

Figure 8.11 - Simulated reflectivity for samples with (a) different ITO thicknesses from 0 to 300 nm, (b) $170 \mathrm{~nm}$ ITO and different $\mathrm{MoO}_{3}$ thicknesses from 0 to $40 \mathrm{~nm}$, (c) $170 \mathrm{~nm}$ ITO, $20 \mathrm{~nm}$ $\mathrm{MoO}_{3}$ and different $\mathrm{CuPc}$ thicknesses from 0 to $70 \mathrm{~nm}$, (d) $170 \mathrm{~nm}$ ITO, $20 \mathrm{~nm} \mathrm{MoO}, 30 \mathrm{~nm}$ $\mathrm{CuPc}$ and different PTCDI-C8 thicknesses from 0 to $70 \mathrm{~nm}$. 


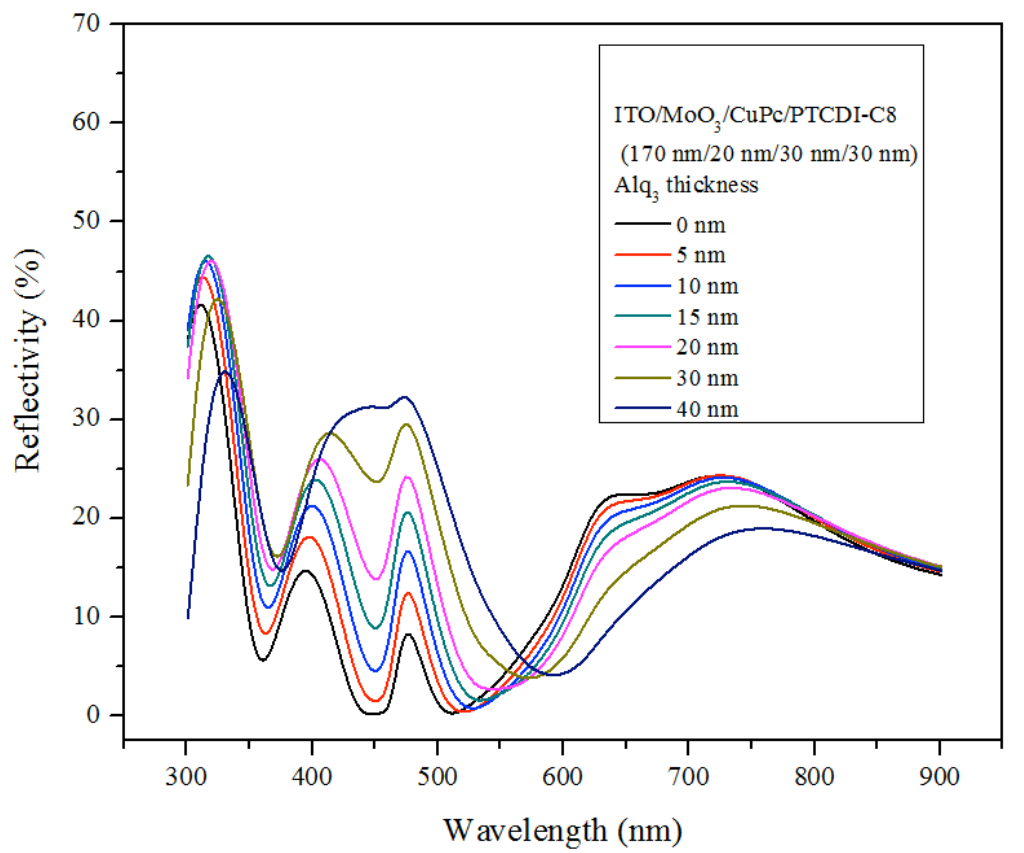

Figure 8.12 - Simulated reflectivity for the structures with $170 \mathrm{~nm}$ ITO, $20 \mathrm{~nm} \mathrm{MoO}_{3}, 30 \mathrm{~nm}$ $\mathrm{CuPc}, 30 \mathrm{~nm}$ PTCDI-C8 and different $\mathrm{Alq}_{3}$ thicknesses from 0 to $40 \mathrm{~nm}$.

compared to the simulation results. A summary of the simulation and the experimental results for these structures are presented in the Figures 8.13 and 8.14. As can be seen in these figures, the experimental results verify presented reflectivity simulation results.

Based on the results of the reflectivity simulations, the HTL reflectivity data indicate that devices with thin HTL $\left(\mathrm{MoO}_{3}\right)$ have a lower reflectivity effect and are more desirable because they allow most of the absorbed light to reach the interface between the two active layers where charge separation occurs. Analyzing reflectivity simulation data of the active layers (CuPc and PTCDI-C8) requires accounting for several additional optical and electrical parameters in order to optimize the efficiency of the device. In a general sense, on one level, more light will be 
absorbed in solar cells with thicker absorber materials. However, as mentioned before in this work, fabricating devices with thicker absorber materials brings significant cost increase. Therefore generally, thin film solar cells are of much higher interest. On another level, organic materials due to their high absorption coefficients are a promising group of materials for thin film solar cells. However, for organic materials, factors such as smaller carrier mobility and shorter lifetime compared to the inorganic materials remain significant factors to consider. Moving on to the reflectivity simulation data, there exists a $25 \%$ to $30 \%$ reflectivity for the different thicknesses of active layers (CuPC and PTCDI-C8), which results in light absorption loss. However, adding ETL $\left(\mathrm{Alq}_{3}\right)$ to the device structure will increase the efficiency due to electrical and optical modifications. An optimized thickness of ETL layer will block damage to the active layer from the metal electrode (Al) deposition, and at the same time will increase reflectivity and will bring back absorbed light to the interface between the two active layers where charge separation occurs. Electrical characterization of the fabricated solar cells has shown an increase in the short circuit current that results in higher efficiency for devices with thicker ETL. To summarize, for the fabricated devices in this work, a thin HTL and a relatively thick ETL should be added to the thin active layers in the fabrication of solar cells. 


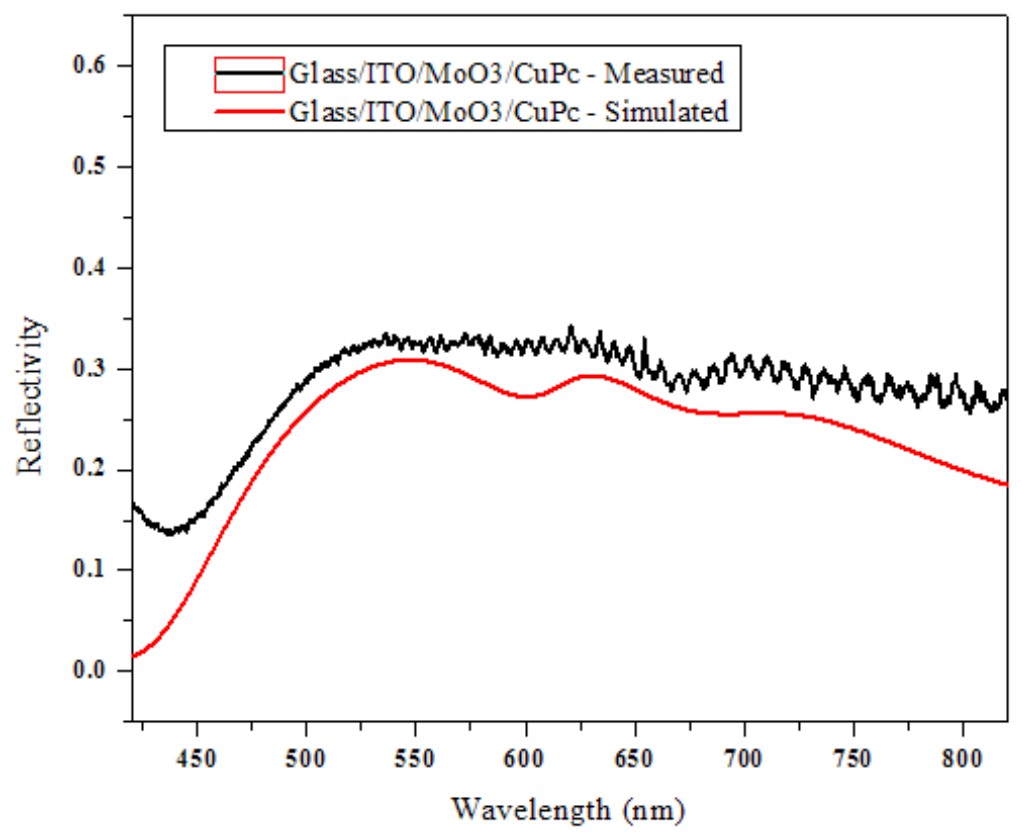

Figure 8.13 - Simulated and experimental reflectivity for the following structure: ITO (170nm) $/ \mathrm{MoO}_{3}(30 \mathrm{~nm}) / \mathrm{CuPc}(40 \mathrm{~nm})$.

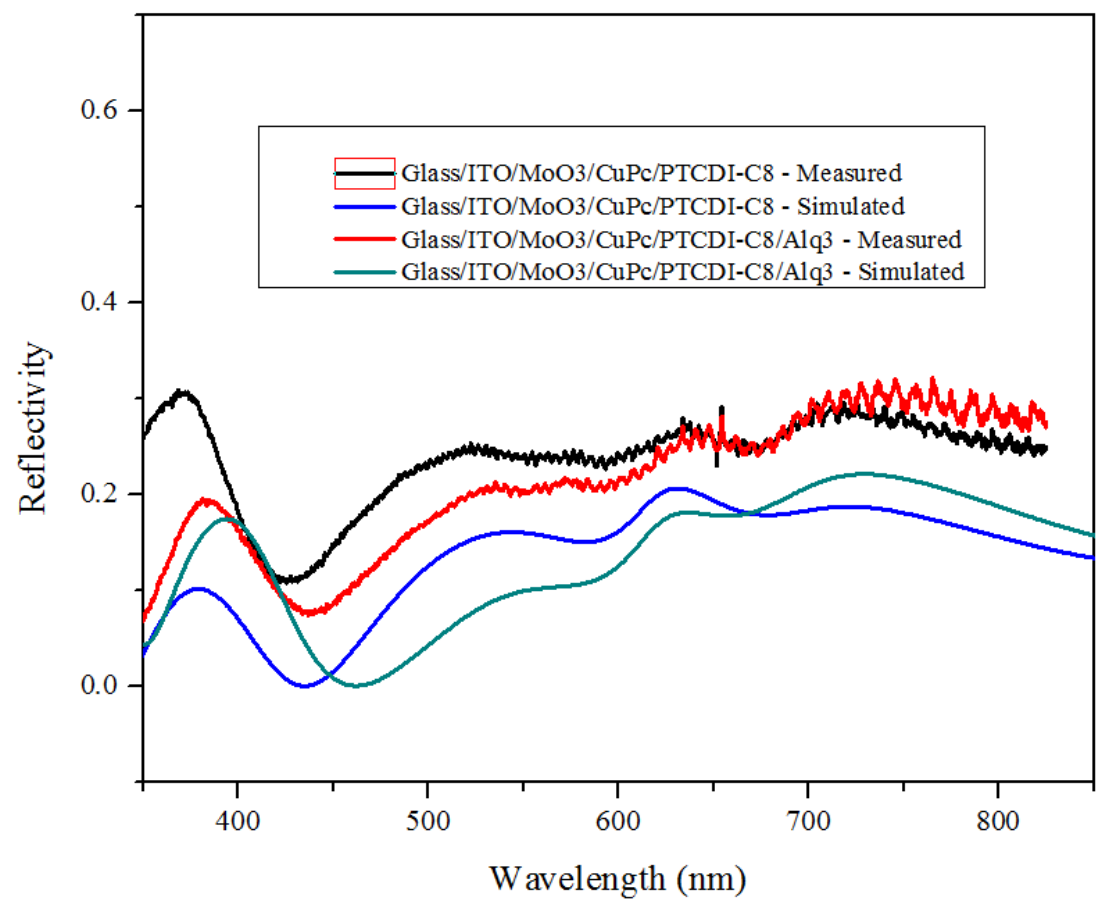

Figure 8.14 - Simulated and experimental reflectivity for the following structure: ITO (170nm) $/ \mathrm{MoO}_{3}(20 \mathrm{~nm}) / \mathrm{CuPc}(30 \mathrm{~nm}) / \mathrm{PTCDI}-\mathrm{C} 8(30 \mathrm{~nm}) / \mathrm{Alq}_{3}(20 \mathrm{~nm})$. 


\subsubsection{Organic/organic interface properties}

In order to study the effect of the active layers' structures on the device performance, several $\mathrm{CuPc} / \mathrm{PTCDI}-\mathrm{C} 8$ films with a total thickness of $40 \mathrm{~nm}$ but with different structures such as co-deposited, layered and bilayer were deposited. Films with layered structure were prepared by the periodic deposition of $5 \mathrm{~nm}$ of each organic material up to the total film thickness of 40 $\mathrm{nm}$. For bilayer structures, $20 \mathrm{~nm}$ of CuPc was deposited following by a deposition of a $20 \mathrm{~nm}$ of PTCDI-C8. Lastly, a co-deposition of CuPc and PTCDI-C8 with the ratio of 1:1 was performed in order to prepare the co-deposited films studied in this work.

The structural properties of these films were studied using X-ray diffraction. Solar cell devices based on these structures were fabricated and their electrical characteristics were studied. The correlation between active layers' structures and the electrical characteristics of the devices has been explored and the results are presented in this section. Figures 8.15 (a), 8.15 (b) and 8.16 show the measured and simulated X-ray patterns for the films with $\mathrm{CuPc} / \mathrm{PTCDI}-\mathrm{C} 8$ bilayer, layered and co-deposited structures, respectively. For bilayer structures, strong diffraction peaks at $4.3^{\circ}$ and $6.8^{\circ}$ were observed. These diffraction peaks are associated with the PTCDI-C 8 and $\mathrm{CuPc}$ material, respectively. The d-spacing of $2.1 \mathrm{~nm}$ and $1.38 \mathrm{~nm}$ corresponding to the (001) plane [31] of PTCDI-C8 and CuPc were calculated from X-ray patterns. However, for the films with the layered structure, a single diffraction peak at $2 \theta=6.8^{\circ}$ corresponding to $\mathrm{CuPc}$ was observed. Kiessig fringes were observed in the X-ray data for the co-deposited films (Figure 7.27). From these fringes, the total thickness of the film was calculated, which was close to the targeted thickness of $40 \mathrm{~nm}$. In the co-deposited structure with a mixture of the donor and the acceptor materials, the interface between the two organic materials is distributed over the entire 
blended film. However, there is a very low control over this distribution. Such distributed interfaces might affect the transport efficiency of both types of the charge carriers to the
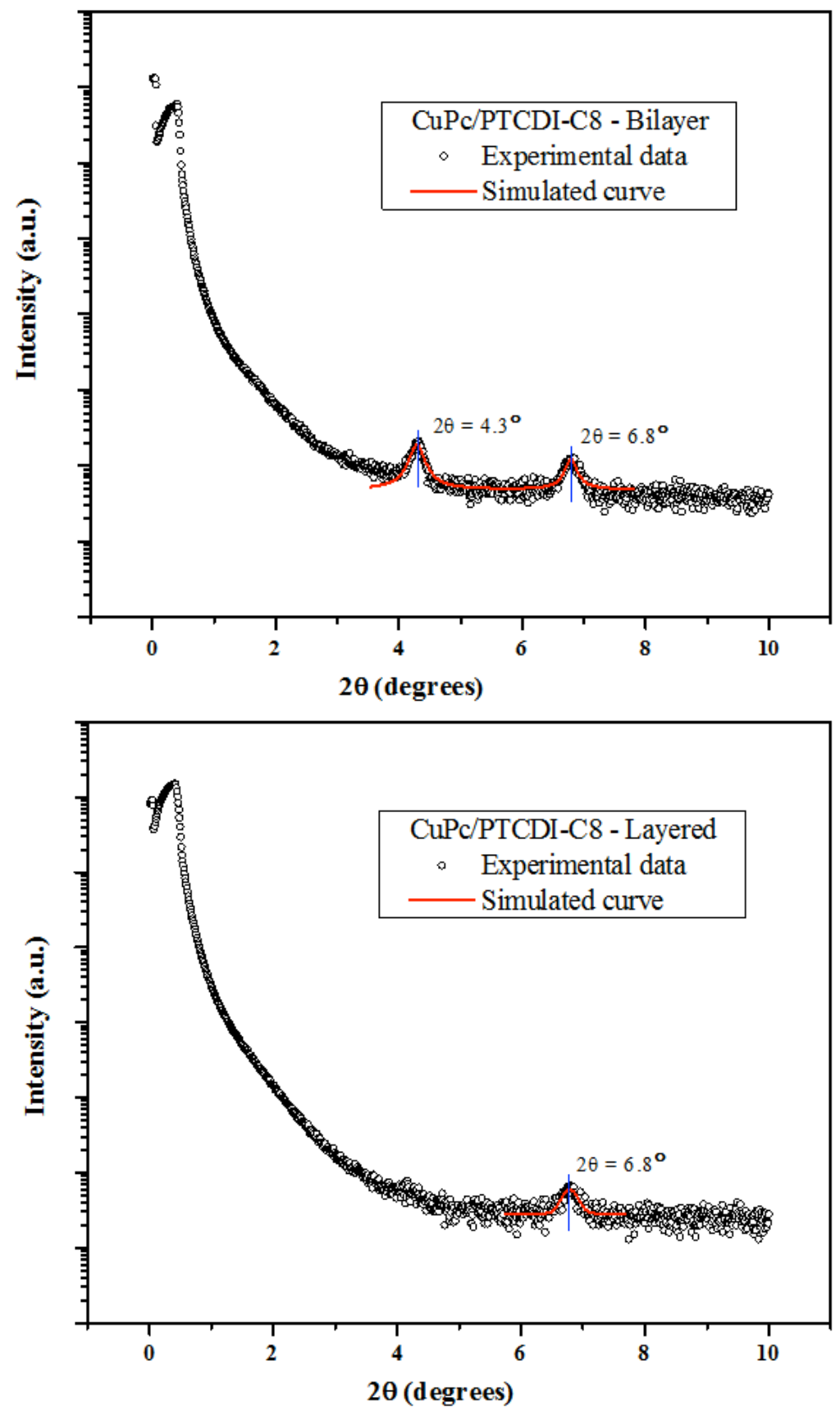

Figure 8.15 - Simulated and experimental XRR data for (a) bilayer and (b) layered CuPC/PTCDI-C8 films. 


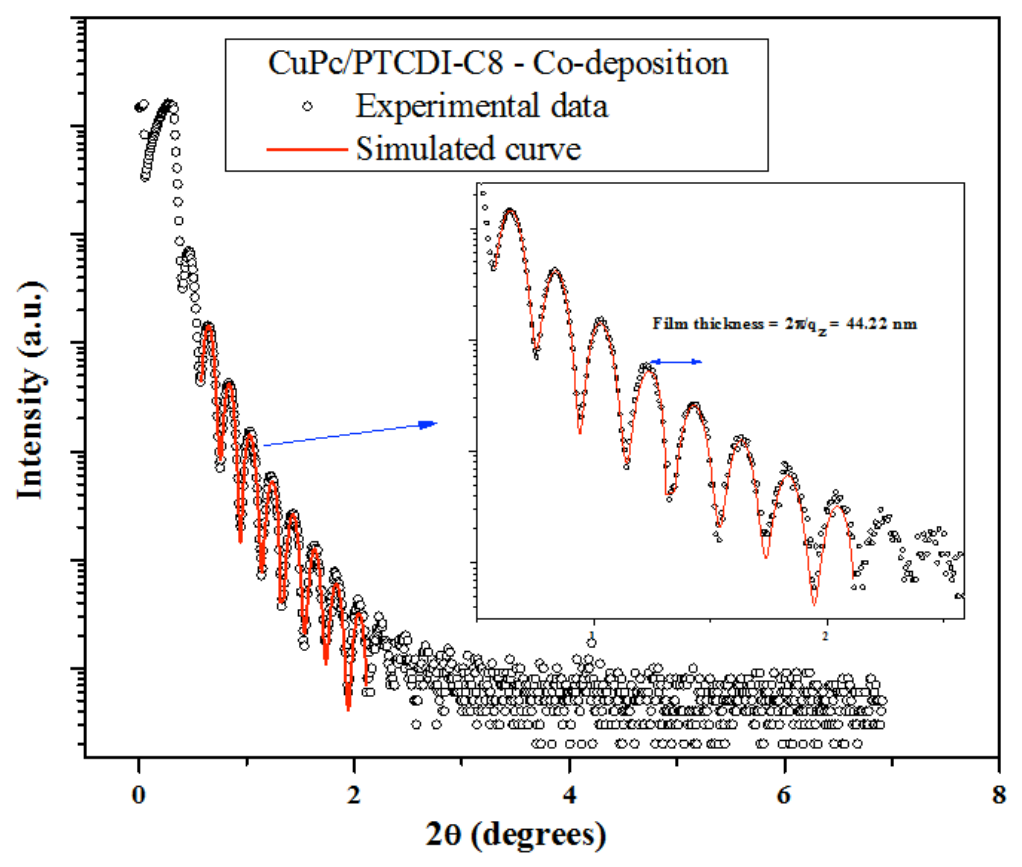

Figure 8.16 - Simulated and experimental XRR data for CuPC/PTCDI-C8 co-deposited film.

electrodes resulting in inferior electrical characteristics. Prominent diffraction peaks for $\mathrm{CuPc}$ and PTCDI-C8 indicate the formation of the higher degree of crystallinity for the films with the bilayer structure compared to the films with layered or co-deposited structures. The crystallinity of the bilayer film results in reduced recombination losses as well as microscopic shorts within the devices. For further analysis, OSCs based on these structures have been fabricated and their $\mathrm{J}-\mathrm{V}$ plots are presented in Figure 8.17. As presented in this figure, devices with bilayer structure exhibit superior electrical characteristics compared to the devices with layered or co-deposited structures of $\mathrm{CuPc} / \mathrm{PTCDI}-\mathrm{C} 8$ films. A summary of the $\mathrm{V}_{\mathrm{OC}}$ and $\mathrm{J}_{\mathrm{SC}}$ of these devices are presented in the Figure 8.18. Devices based on the co-deposited films exhibit lower $\mathrm{J}_{\mathrm{SC}}$ and higher $\mathrm{V}_{\mathrm{OC}}$ due to the amorphous properties of the active layers. Our results are in agreement with the previous reported results $[90,91]$. Organic layers with disordered grains may reduce the 
device performance due to the large number of defect states and recombination losses. These results emphasize the importance of the effect of the active layers' structures on the device performance.

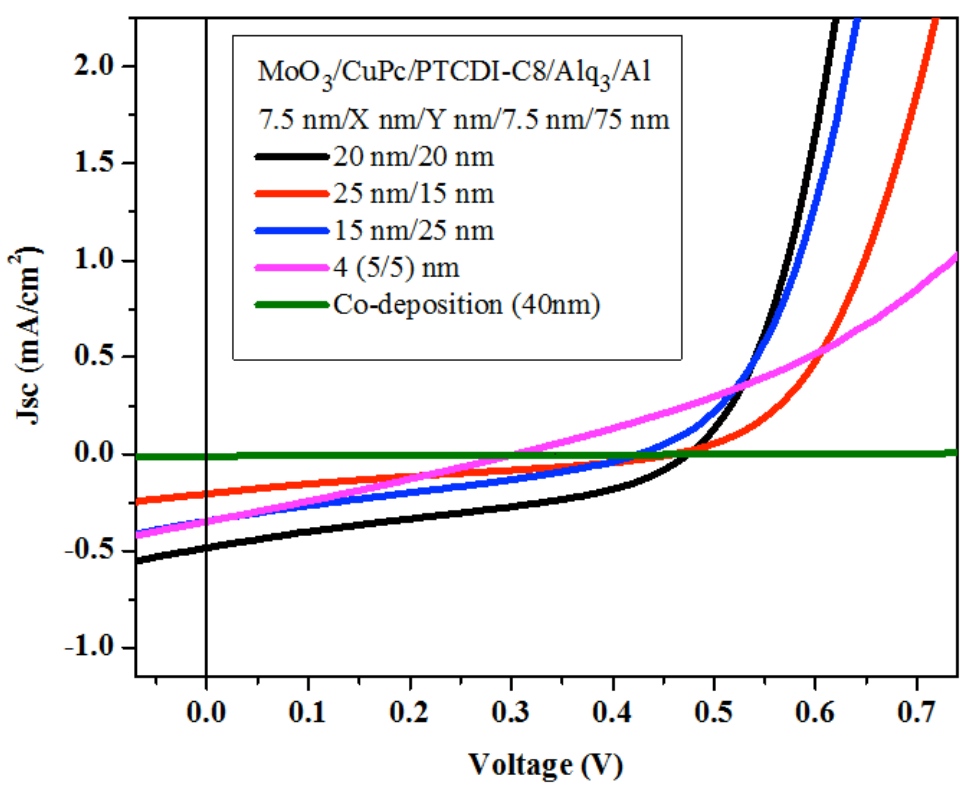

Figure $8.17-\mathrm{J}-\mathrm{V}$ results of OSCs with different active layers structures.
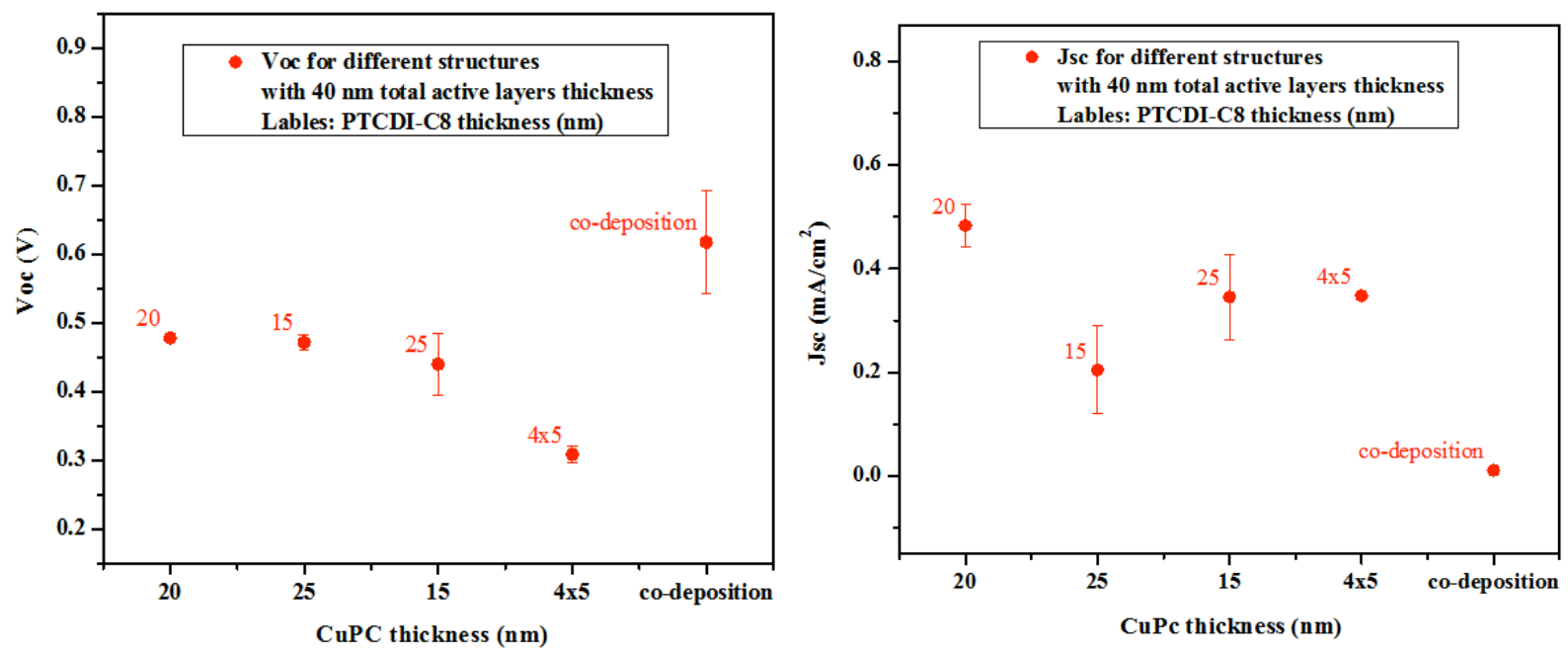

Figure 8.18 - Plots of $\mathrm{V}_{\mathrm{OC}}$ and $\mathrm{J}_{\mathrm{SC}}$ for the OSCs with different active layer structures. 


\subsection{ORGANIC THIN FILM TRANSISTORS}

\subsubsection{Electrical characteristics - ambipolar behavior}

The mechanical flexibility, low weight and low-cost processing of organic materials are some of the main reasons for considering organic materials as valuable alternatives to the more commonly used inorganic materials for applications in electronic devices [91 - 93]. In addition to these factors, organic semiconductor materials exhibit ambipolar charge carrier transport. This interesting feature provides ease of design and ability to fabricate low-cost and flexible electronic devices such as light-emitting field-effect transistors as well as organic complementary metal oxide semiconductor (CMOS) devices. Ambipolar conduction can also provide important information that helps with fundamental understanding of the transport processes in organic materials [94].

Ambipolar transport can be achieved by using blends or bilayers of n-type or p-type organic materials $[91,93]$. This conduction behavior can also be achieved in devices with a single layer organic material by employing a high or a low work function metal, respectively, for hole or electron injection $[95,96]$. Engineering semiconductor-dielectric interface properties by adding an additional layer between organic and dielectric layer is another possible way to fabricate ambipolar organic transistors [97]. Generally, charge transport in organic semiconductor materials are affected by their disordered structure that results in randomly distributed large density of trap states that can significantly change the electrical behavior of the fabricated structures [98]. In this work, it has been shown that ambipolar transport can be achieved within a single transistor channel using $\mathrm{LiF}$ gate dielectric in the transistors with pentacene active layer. This ambipolar behavior can be controlled by the applied source-drain 
and gate biases. The observed trends in the electrical behavior of these structures are well described by the multistep hopping and Fowler-Nordheim (F-N) tunneling mechanis.

Figure 8.19 shows the output characteristics of the fabricated OTFTs under different negative and positive gate voltages. Under negative gate voltages, when the transistors are operating as p-type devices, the output current is about an order of magnitude larger compared to the current at the positive gate voltages. Trapping of electrons at the semiconductor-dielectric interface, resulting in less mobile carriers for n-type behavior, has been suggested to be the reason for the observed I-V characteristics [32]. F-N theory and the corresponding equation (Eq.8.4) have been used to analyze the $\mathrm{I}-\mathrm{V}$ results at each applied gate bias. $\mathrm{J}$ is the field emission current; $\varphi$ is the work function; $\mathrm{E}=\beta \mathrm{V}$ represents the applied electric field; and $\beta$ is the field enhancement factor which is defined as the ratio of the local surface electric field to the applied bias [99].
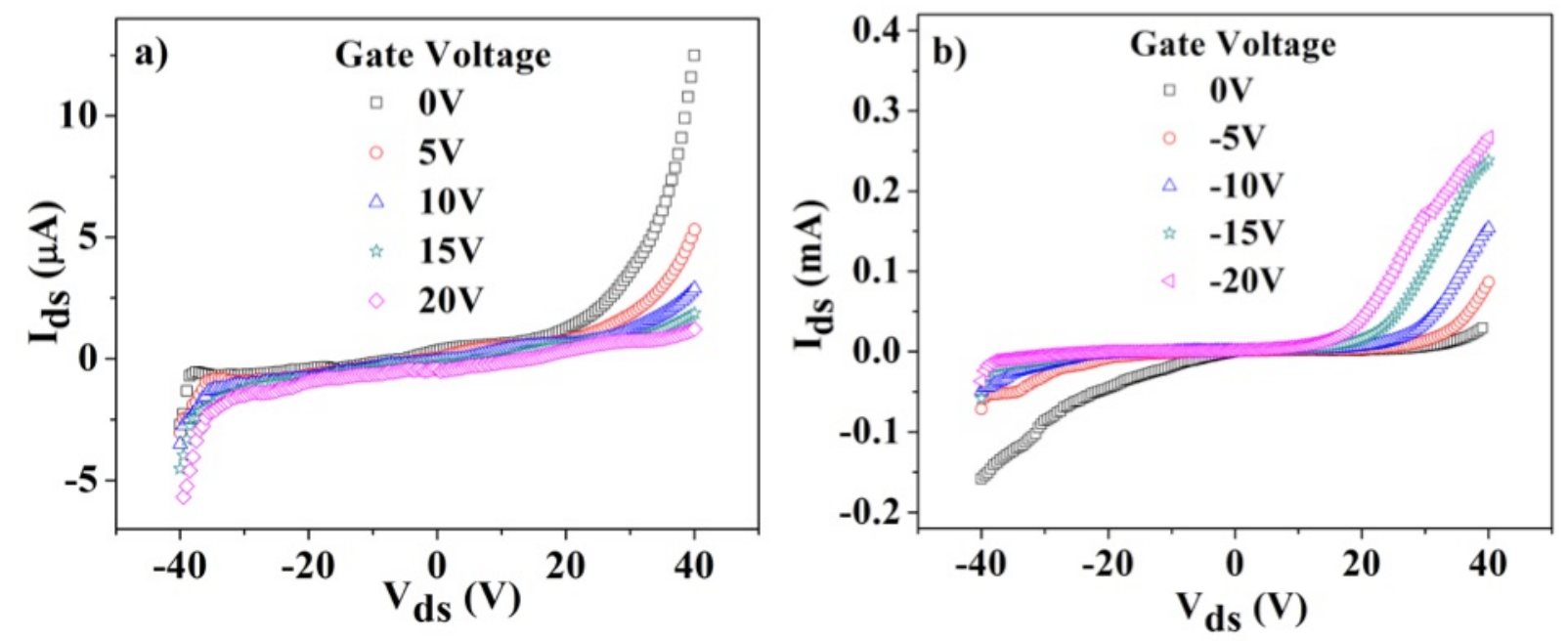

Figure 8.19 - Plot of drain-source current (Ids) vs. drain-source voltage (Vds) for different (a) positive and (b) negative gate voltages in the top-contacted pentacene-LiF ambipolar thin film transistor. 
$J=1.54 \times 10^{-6} \frac{E^{2}}{\varphi} \exp \left[-6.83 \times 10^{7} \frac{\varphi^{3 / 2}}{E}\right]$

Figure 8.20 shows the F-N plots for negative and positive gate voltages. These plots have been extracted from the I-V results. Least-square error fitting was utilized to determine the slopes of the F-N plots which correspond to $\left(\varphi^{3 / 2} / \beta\right)$. The insets of Figure 8.20 show the leastsquare error fit in the tunneling region for the gate voltage at $\pm 15 \mathrm{~V}$. In the F-N plots, under zero gate voltage, for reverse drain-source biases (Figure 8.20 (a)), as the applied drain-source voltage decreases, the ambipolar transport behavior can be observed. These results suggests that the transport mechanism switches from electron tunneling to electron hopping and then to hole tunneling. On the other hand, for the forward drain-source biases (Figure 8.20 (b)), in order to observe the charge carrier transition from hole hopping to electron tunneling, higher drain-source voltages are required. Since all of the I-V data for this work is taken in the range of -40 to $40 \mathrm{~V}$ for drain-source voltages, the transition in the charge carrier for forward biases cannot be presented in the corresponding F-N plots.

The dependency of the field enhancement factor and the work function on the applied gate bias was shown by calculating the slopes of the tunneling region. The value of $\beta$ is related to the geometry, crystal structure, surface morphology and electrical homogeneity [100, 101]. Although tunneling current is known to be affected by the field enhancement factor as well as the work function, as the effects of these two parameters are coupled together, it is difficult to analyze the F-N current based solely on one or the other [99]. The drain-source voltage at which the conduction mechanism switches from hopping to tunneling was extracted for each of the gate biases by calculating the slopes of the hopping and the tunneling regions in the F-N plots. Figures 8.21 (a) and (b) show the dependency of the slope of the F-N plots as a function of the 
gate voltage. As the gate voltages increase, for both negative and positive gate biases, the transition points occur at lower source-drain biases. The slope of the F-N plots as a function of gate voltage is plotted in Figures 8.21 (c) and (d). These figures show that for positive gate voltages, the slope follows $\beta$, while for negative gate voltages, the slope shows more dependency on $\varphi$. These results indicate that the current shows more dependency on the applied gate bias under negative gate voltages. This can be explained based on the Fermi level pinning occurring at the dielectric-semiconductor interface due to the trap levels $[98,102]$. Under negative gate biases, trap levels at the dielectric-organic interface are neutralized by the anions (F- in LiF) $[103,104]$, resulting in suppression of Fermi level pinning and a higher dependency of the $\varphi$ on the applied gate bias. However, trap levels are not neutralized for positive gate biases, which results in less dependency between $\varphi$ and the gate voltage.

In order to further understand the transport mechanisms in the fabricated structures, a thin layer of $\mathrm{V}_{2} \mathrm{O}_{5}$ as an interface layer was introduced between the gate dielectric and organic layers. As expected, due to the effect of the interface layer, a significant decrease in the amount of the source-drain current was observed. For devices without the interface layer, the output currents are about two orders of magnitude higher compared to the devices with the interface layer. Ambipolar transport behavior in pentacene-based organic thin film transistors with $\mathrm{LiF}$ gate dielectric has been studied. It has been shown that at low source-drain voltages, multistep hopping is the dominant transport mechanism, whereas electrical characteristics fit the FowlerNordheim (F-N) tunneling model for higher source-drain voltages. F-N plots have been used to show the dependency between field enhancement factor and the transition point in conduction mechanism upon gate bias. 

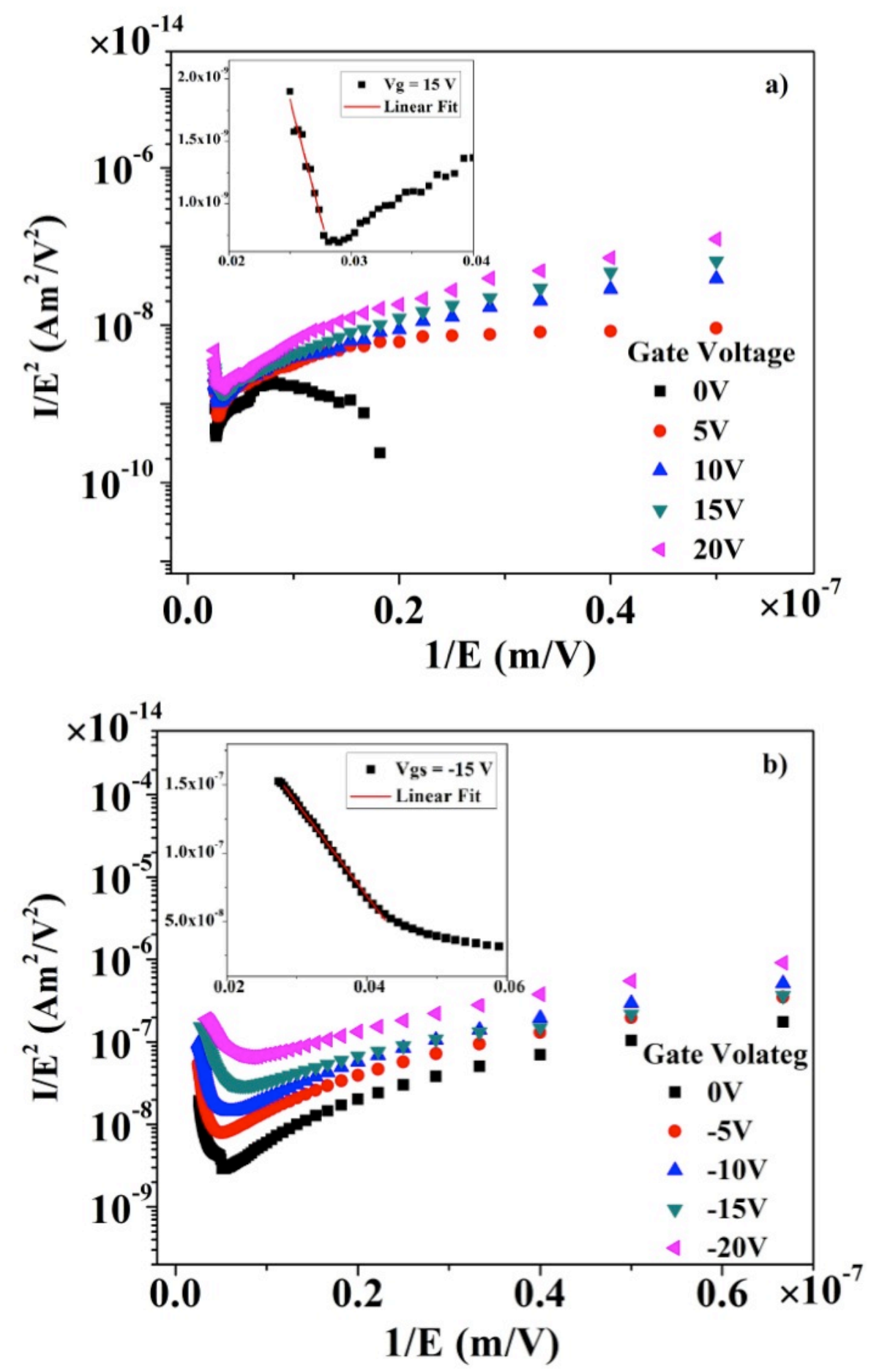

Figure 8.20 - The corresponding Fowler-Nordheim (ln (I/E2) vs. 1/E) plots for (a) positive, and (b) negative gate voltages. The insets show the least-squares error fit in the tunneling region for gate voltages at $\pm 15 \mathrm{~V}$. 

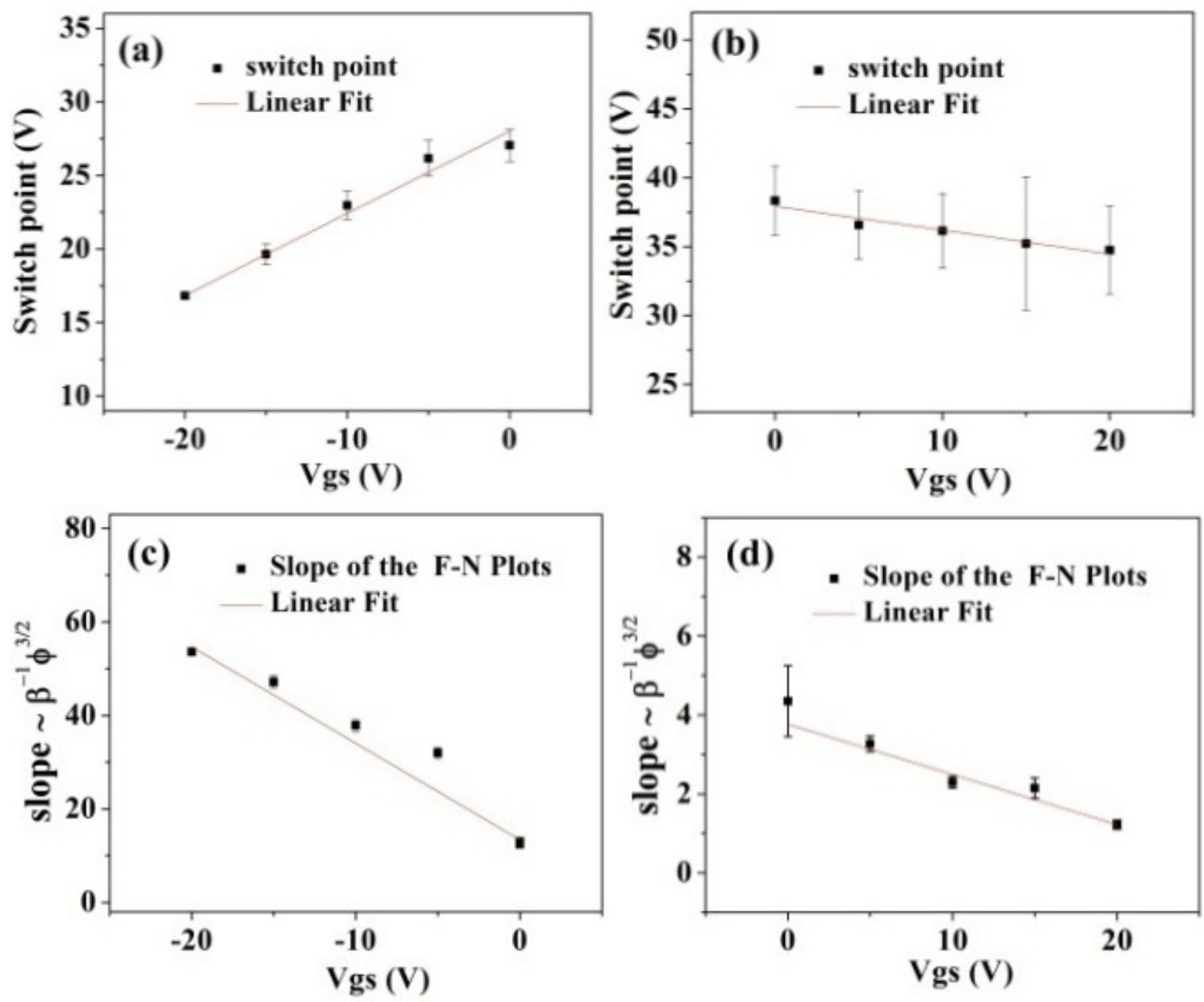

Figure 8.21 - Plot of the source-drain voltage, at which transition from hopping to tunneling occurs, vs (a) negative and (b) positive gate voltages. The gate-voltage dependence of the $\beta$ and $\varphi$ in Fowler-Nordheim law calculated from the slope of the tunneling regions in FN plots for (c) negative and (d) positive gate voltages.

The results of this study suggest that the charge transport behavior in OTFTs, and perhaps in other organic semiconductor devices in general, correlate not only with the organic semiconductor film structure, but also with other parameters such as barrier height at the metalsemiconductor interface, carrier traps at the dielectric-semiconductor interface and trap generation due to exposure to different environments. 


\subsubsection{Device structure effects - doping and photosensitivity}

Several samples were fabricated based on pentacene and PTCDI-C8 as organic materials.

Each sample was characterized at room temperature and under atmospheric conditions. The effect of light on the electrical properties of the samples was investigated as well. The output characteristics of the fabricated OTFTs described as the drain-source current $\left(\mathrm{I}_{\mathrm{ds}}\right)$ versus the drain-source voltage $\left(\mathrm{V}_{\mathrm{ds}}\right)$ were investigated for varying gate voltages and are shown in Figure 8.22 (a) and (b) for PTCDI-C8 and pentacene, respectively. As seen, with an increase in the gate voltage applied to the device, a relatively stronger field effect modulation of the conduction channel was obtained with the on/off ratio $\left(\mathrm{I}_{\mathrm{on}} / \mathrm{I}_{\mathrm{off}}\right)>10^{3}$ which is comparable to the results with the conventional structures $[105,106]$.
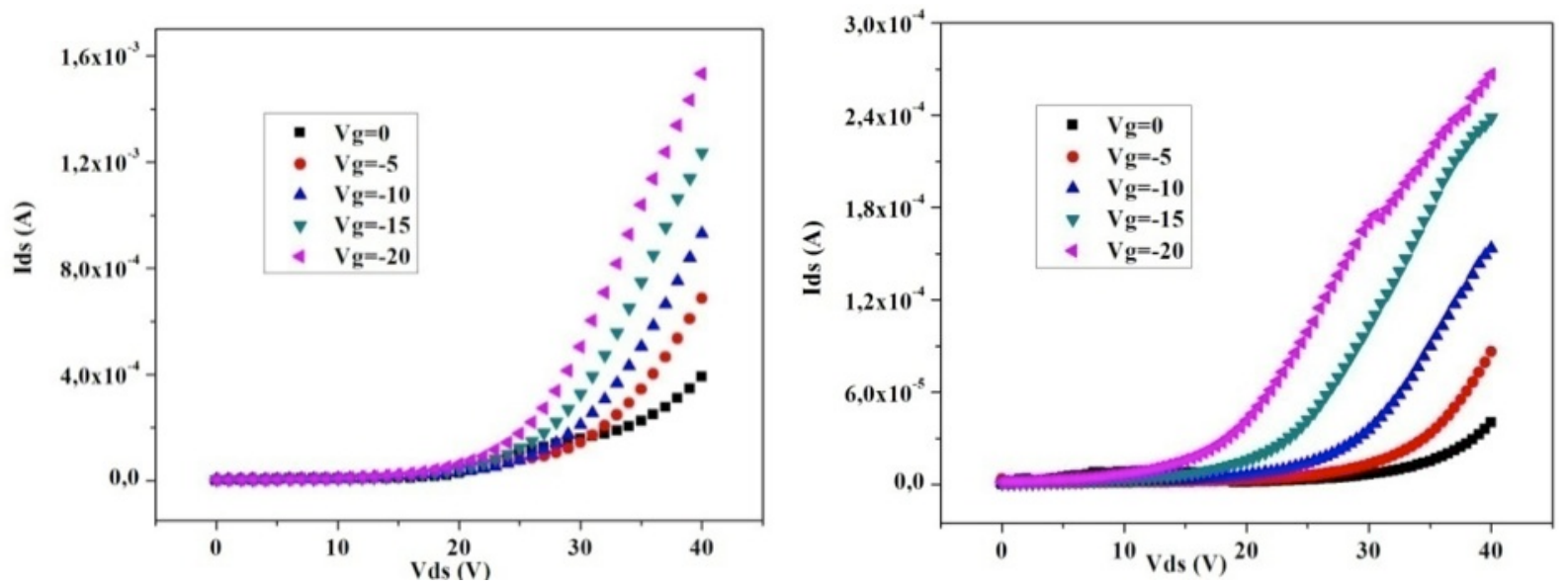

Figure 8.22 - Source-drain current-voltage characteristics of the OTFT with (a) PTCDI-C8 and (b) pentacene as the organic semiconductor materials.

As presented in Figures 8.22 (a) and (b), $\mathrm{I}_{\mathrm{ds}}$ at a constant $\mathrm{V}_{\mathrm{ds}}$ increases with applying higher negative gate voltage. In this work, an operating mechanism for the fabricated transistors with $\mathrm{LiF}$ as the gate dielectric layer is proposed after careful evaluation of the experimental 
results and thorough review of the related literature. This conduction behavior can possibly be attributed to the migration of the $\mathrm{Li}^{+}$and $\mathrm{F}^{-}$ions into the organic semiconductor as well as the doping process of the active layer. According to experimental data from various sources, it has been shown that under atmospheric conditions, $\mathrm{LiF}$ can form the anion $\mathrm{F}^{-}$and the cation $\mathrm{Li}^{+}$ which introduces dopant into organic semiconductors such as pentacene, PTCDI-C8 and P3HT $[103,104,107]$. For the fabricated devices in this work, it is believed that the ion drifting and doping process modifies the carrier injection barrier between the dielectric, semiconductor layer and the source-drain electrodes. The created anions and cations will be separated by the induced electric field inside the gate dielectric layer due to the applied gate voltage. These ions will diffuse into the organic semiconductor, here pentacene or PTCDI-C8, and serve as dopant that will change the carrier injection barrier between the organic layer and the source-drain electrodes. The separation and drifting directions of the created ions and the doping process can be controlled by the applied gate voltage [108, 109]. This doping process can be studied using Fourier transform infrared (FTIR) spectroscopy and micro-Raman spectroscopy [9, 15]. Kaake et al. has applied FTIR spectroscopy to study the effect of the gate bias on the doping process in the PTCDI-C8 films [108].

The FTIR results show the formation of anionic PTCDI-C8 film from electron injection under positive gate biases. For the fabricated pentacene transistors in our work, Raman spectroscopy under various applied gate voltages has been performed and the results present changes in the intensity of the peaks attributed to the bonding inside pentacene. Figure 8.23 shows the typical Raman spectrum for pentacene films. The observed peaks at 1158 and 1178 $\mathrm{cm}^{-1}$ are related to $\mathrm{C}-\mathrm{H}$ in-plane bending modes, and the 1353,1371 and $1379 \mathrm{~cm}^{-1}$ peak belong to the aromatic $\mathrm{C}-\mathrm{C}$ stretching modes $[9,15]$. The intensity of the observed peaks decreases as 
the applied positive gate biases increase, indicating the effect of the doping process on the intermolecular coupling between the pentacene molecules. Introducing more $\mathrm{Li}+$ dopants for positive gate biases decreases the intermolecular coupling that results in lower intensity of the Raman peaks.

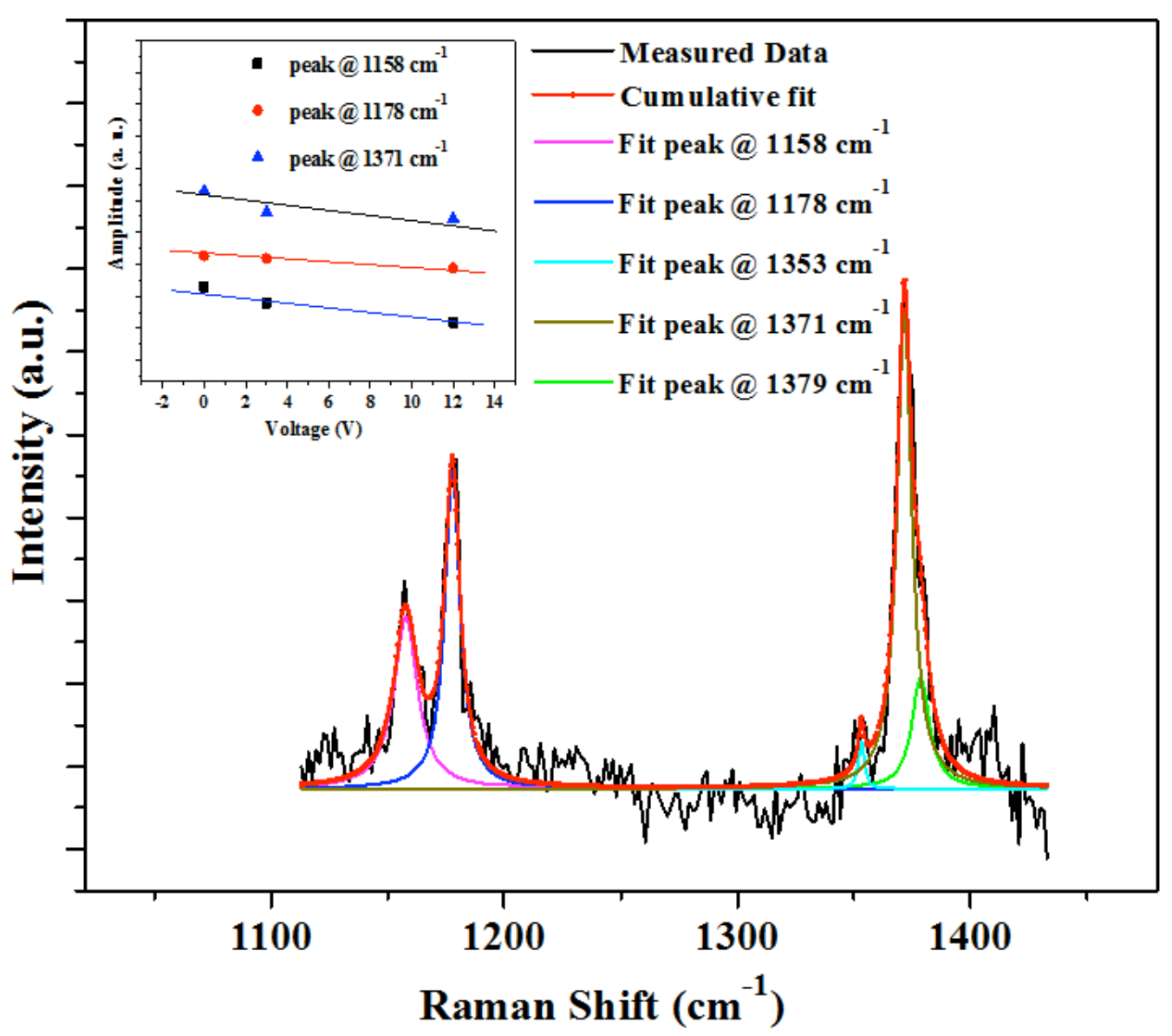

Figure 8.23 - Raman spectrum with the fitted peaks for pentacene films. The insets show the effect of gate bias on the Raman peaks.

Figure 8.24 shows the relationship between drain current and the thickness of the channel. By increasing the thickness of the organic semiconductor material, the amount of the drain- 
source current increases due to the availability of more charge carriers in the channel. Figure 8.25 presents $\mathrm{I}_{\mathrm{ds}}$ as a function of organic layer thickness for three OTFTs with pentacene

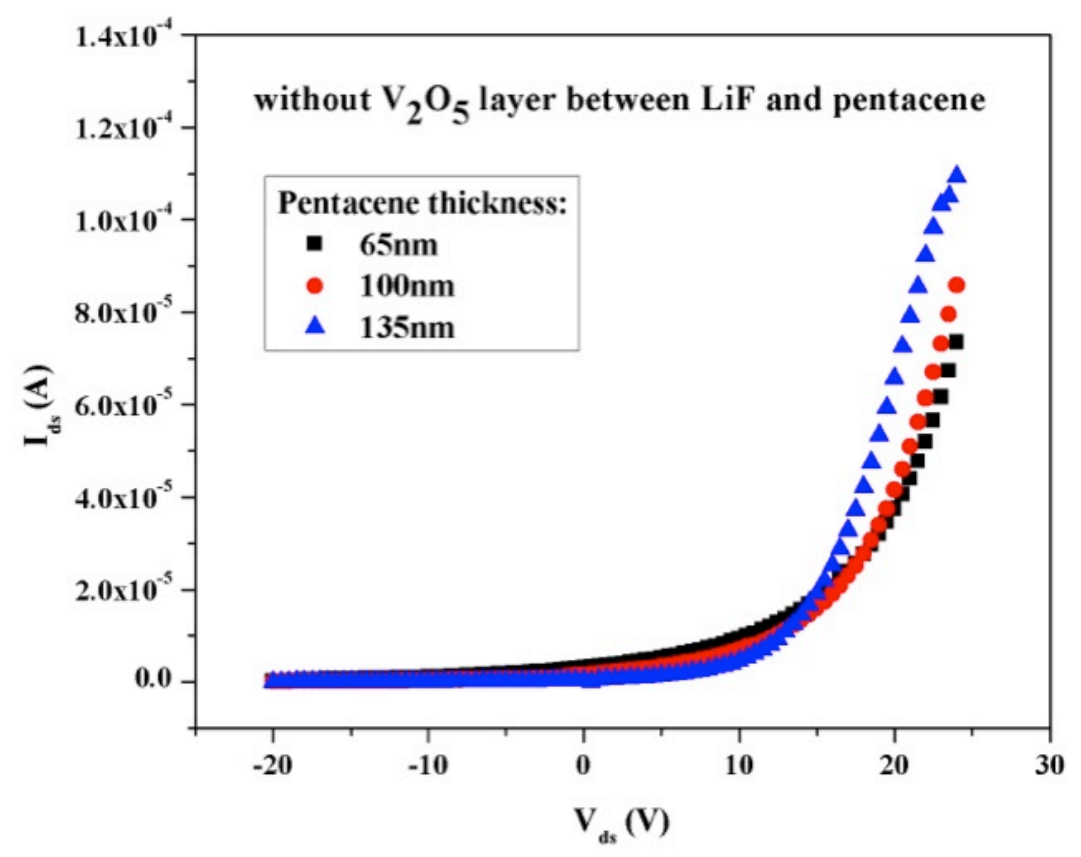

Figure 8.24 - Source-drain I-V characteristics of the OTFT without $\mathrm{V}_{2} \mathrm{O}_{5}$ layer between $\mathrm{LiF}$ and pentacene with different thickness of pentacene $(65 \mathrm{~nm}, 100 \mathrm{~nm}, 135 \mathrm{~nm})$ at $\mathrm{Vg}=-20 \mathrm{~V}$.

thicknesses of $65 \mathrm{~nm}, 100 \mathrm{~nm}, 135 \mathrm{~nm}$ at $\mathrm{V}_{\mathrm{ds}}=10 \mathrm{~V}$ and $25 \mathrm{~V}$. These data have been extracted from Figure 8.24. As can be seen in this plot, for lower voltages up to $15 \mathrm{~V}$, as the thickness of the pentacene layer increases, the channel current decreases. At higher voltages, however, the opposite trend exists and the channel current increases as the organic layer thickness increases. These results indicate that there are different mechanisms that control drain-source current. In order to further investigate the effect of $\mathrm{LiF}$ as the gate insulating layer and the doping process of the organic layer due to $\mathrm{LiF}$, new devices have been fabricated and characterized. These transistors have the same structure as the previous ones except for inserting a thin layer of $\mathrm{V}_{2} \mathrm{O}_{5}$ 
between $\mathrm{LiF}$ and the organic layer. It is believed that this layer will confine the created dopant, which leads to lower conductivity of the channel. Figure 8.26 shows the $I_{d s}$ versus $V_{d s}$ for two different thicknesses of pentacene $(65 \mathrm{~nm}$ and 100 nm) with and without the $\mathrm{V}_{2} \mathrm{O}_{5}$ layer between $\mathrm{LiF}$ and pentacene. Figure 8.27 shows the $\mathrm{I}_{\mathrm{ds}}$ versus $\mathrm{V}_{\mathrm{ds}}$ for the devices with $\mathrm{V}_{2} \mathrm{O}_{5}$ layer between $\mathrm{LiF}$ and pentacene for two different organic layer thicknesses (65 $\mathrm{nm}$ and $100 \mathrm{~nm})$. As can be seen in these figures, the amount of the current decreases

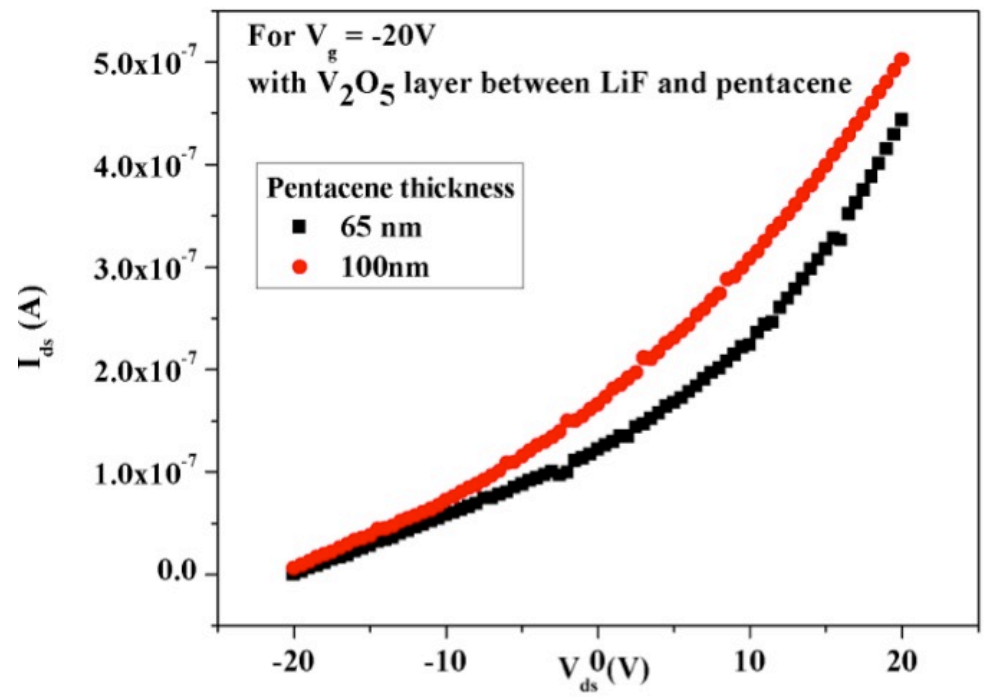

Figure 8.27 - Source-drain current-voltage characteristics of the transistors with $\mathrm{V}_{2} \mathrm{O}_{5}$ layer between $\mathrm{LiF}$ and pentacene with different thicknesses of pentacene at $\mathrm{Vg}=-20 \mathrm{~V}$. Drain current drops significantly compared to the current for the transistors without this layer. 
significantly for the transistors with the $\mathrm{V}_{2} \mathrm{O}_{5}$ layer compared to those without this layer. By inserting this layer, the generated cations and anions in the LiF will be confined in this layer, resulting in less dopant migration into the organic layer.

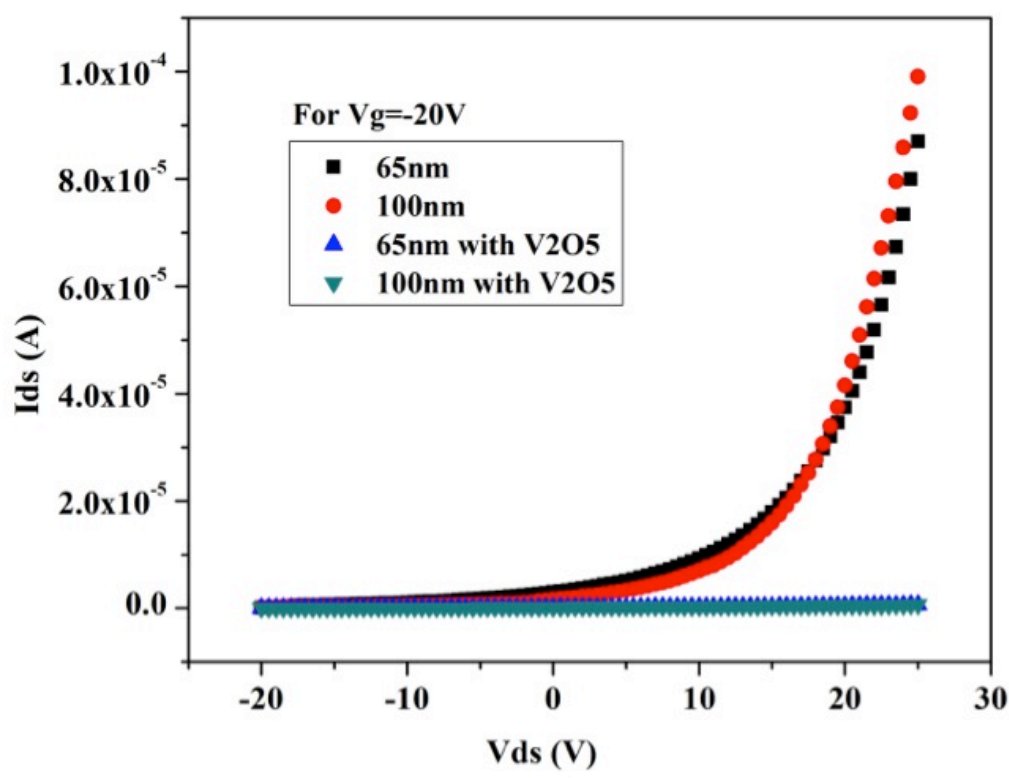

Figure 8.26 - Source-drain current-voltage characteristics of the transistors with and without $\mathrm{V}_{2} \mathrm{O}_{5}$ layer between $\mathrm{LiF}$ and pentacene with different thicknesses of pentacene at $\mathrm{Vg}=-20 \mathrm{~V}$.

The photosensitivity behavior of the fabricated devices has been studied as well and the results are shown in Figures 8.28 (a) and (b) for PTCDI-C8 and pentacene, respectively. One of the interesting applications of the OTFTs is in the organic photo-transistors (OPTs) that combine the detection of the light and the amplifying of the detected signal in a single device. OPTs can be fabricated by solution process or thermal evaporation methods using different organic materials. Pentacene and PTCDI-C8 are among the organic materials that have been found to be highly photosensitive [110]. 

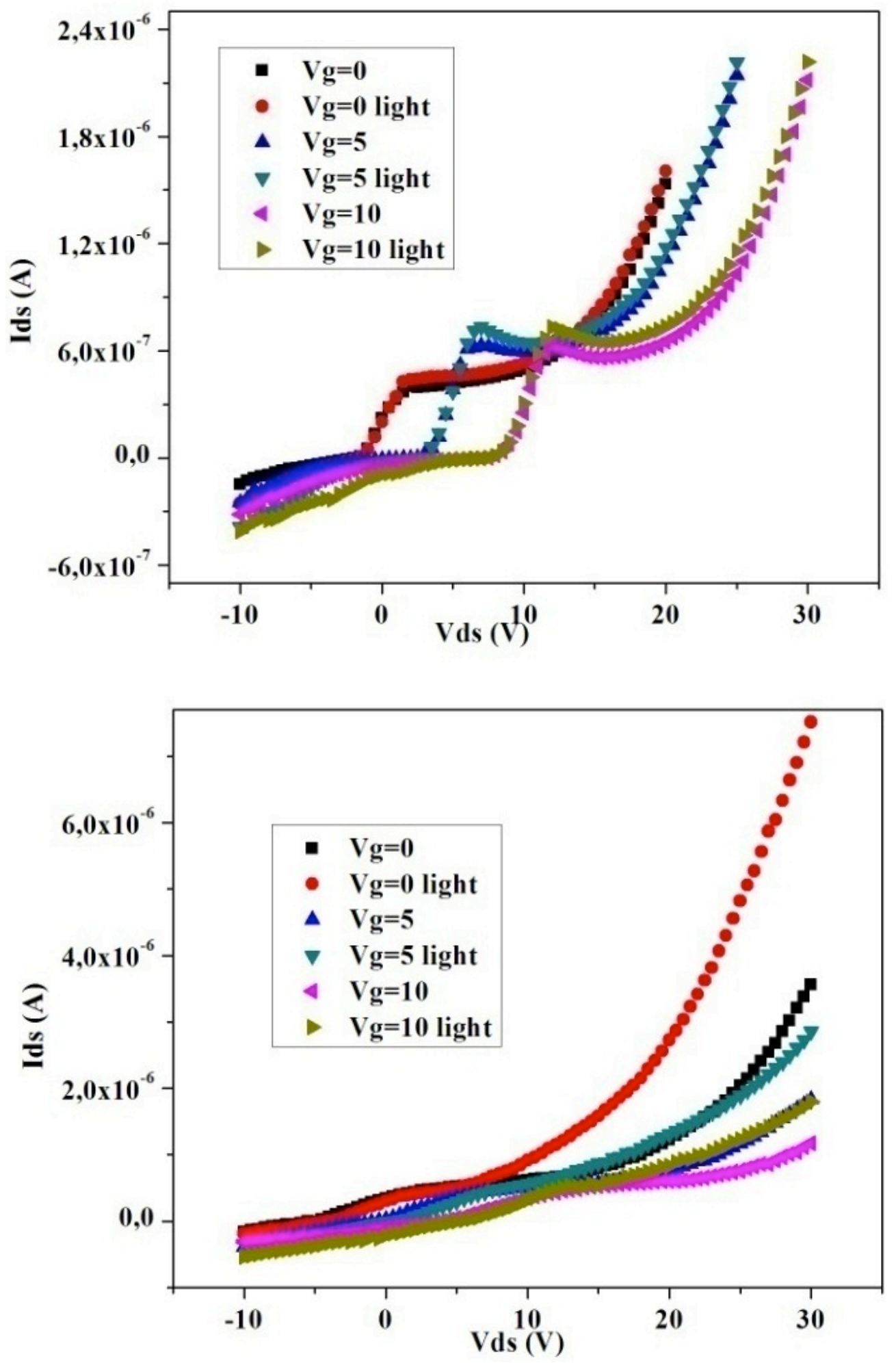
Figure 8.28 - The effect of light on the output characteristics of the transistor with (a) PTCDI-C8 and (b) pentacene as the semiconductor layer at different gate voltages.

A drain-source current increase and a threshold voltage shift upon illumination were observed for the evaluated devices. The current increment is due to the photogeneration of the electron-hole pairs in the channel [33]. Under illumination of light with a wavelength matching the organic semiconductor absorption spectrum, excitons or electron-hole pairs are generated in the channel of the transistor. Photons with lower energy than the optical band-gap of the organic material are absorbed weakly and generate excitons that will dissociate into electron-hole pairs $[110,111]$. Created electron-hole pairs due to the dissociation of excitons or the generated electron-hole pairs due to the absorption of high energy photons will drift in the channel under the source-drain bias, contributing to the drain current. However, some of the photogenerated charge carriers will not contribute to the drain current due to the interaction with each other and recombination. This is more pronounced when the gate voltage increases, and more charge carriers are generated in the channel that results in more recombination and slower rate of increase in the drain current. Figure 8.29 presents the change of the drain current under illumination as a function of the gate voltage for the transistors with pentacene as the organic layer. This structure shows more photosensitivity compared to the transistors with PTCDI-C8. The effect of the light on the current of the channel decreases when higher gate voltages are applied, a phenomenon that is due to higher generation and recombination of the charge carriers [111]. 


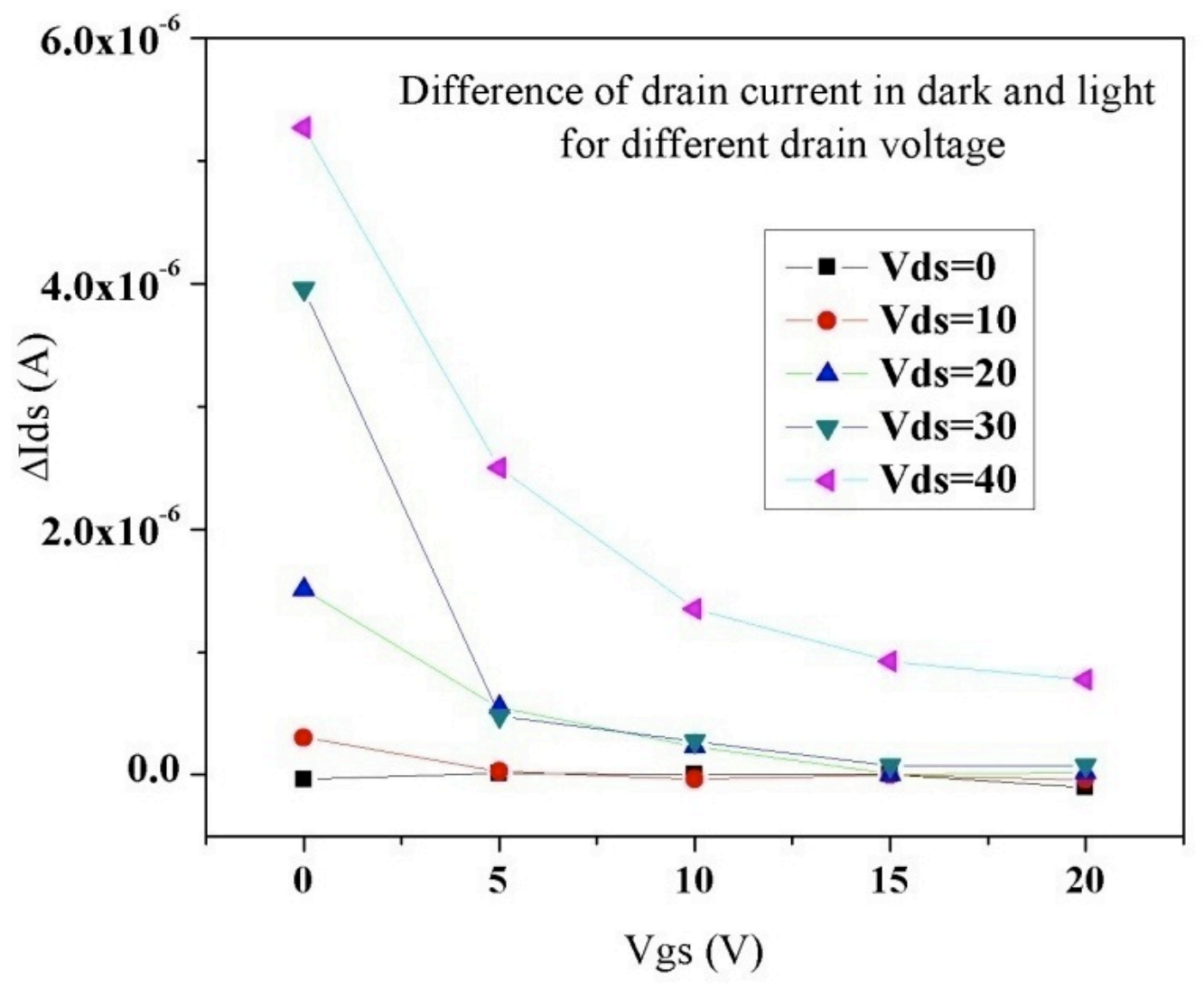

Figure 8.29 - Change of the $\mathrm{I}_{\mathrm{ds}}$ under illumination versus gate voltage for the pentecene-based transistors.

\subsubsection{Dielectric/organic interfacial effects}

Several bilayer structures of $\mathrm{LiF} / \mathrm{PTCDI}-\mathrm{C} 8$ and $\mathrm{LiF} /$ pentacene were prepared and their morphology and molecular structure were characterized using XRR technique. In order to study the effects of the films' structures and dielectric/organic interfacial properties on the device performance, organic thin film transistors based on these bilayers were fabricated and characterized. The fabrication process and the schematic structure of the fabricated devices have been presented in Chapter 6. 
Figure 8.30 shows the measured and simulated XRR data for the LiF/PTCDI-C8 and $\mathrm{LiF} /$ pentacene bilayers with different LiF thicknesses. The X-ray curves have been shifted for clarity. The reflectivity profiles for the LiF/PTCDI-C8 bilayers exhibit clear fringe modulations with two $\Delta$ qs. It implies that PTCDI-C8 layers maintain their multilayered film structures when deposited on $\mathrm{LiF}$ dielectric layer. However, for the $\mathrm{LiF} /$ pentacene bilayers, Kiessig fringes with a single $\Delta \mathrm{q}$ were observed indicating that these films do not maintain an ordered structure on LiF dielectric layer. Furthermore, for the LiF/PTCDI-C8 films, diffraction peak at $2 \theta=4.2^{\circ}$ corresponding to (001) plane of PTCDI-C8 was observed. The presence of this peak implies the polycrystallinity of the PTCDI-C8 thin films. Figure 8.31 shows the output characteristics of the

- Bilaveres

- $\underline{\mathrm{LiF} / \mathrm{PTCDI}-\mathrm{CB}}$

- LiF/Pentacene

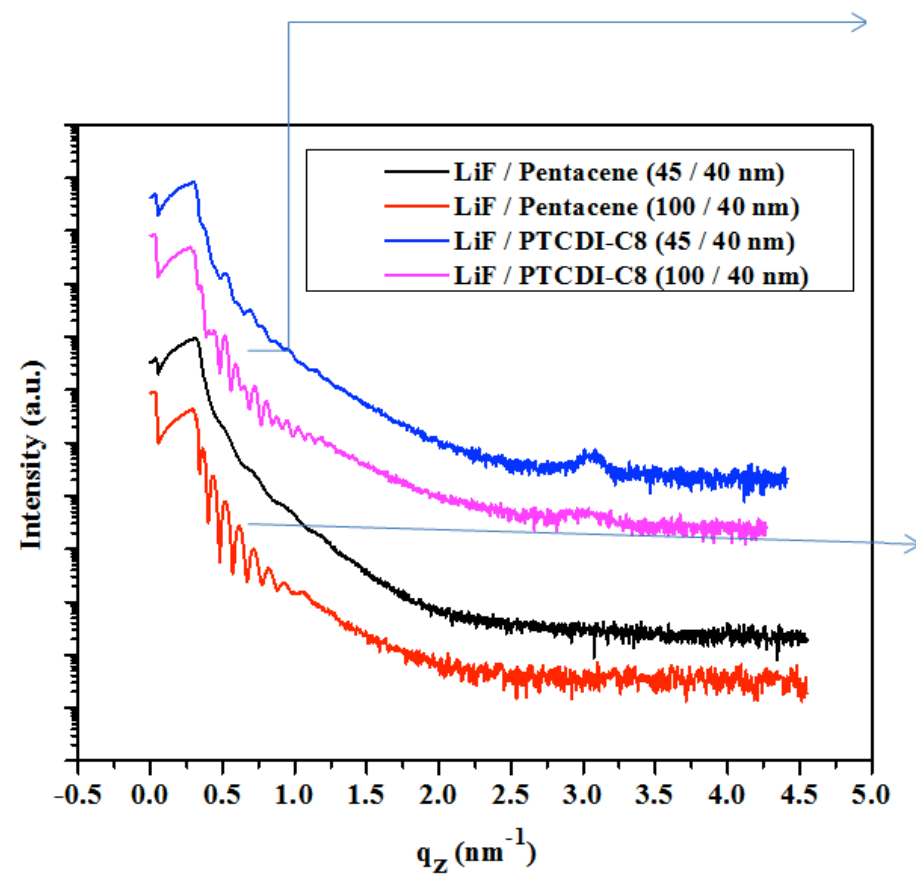

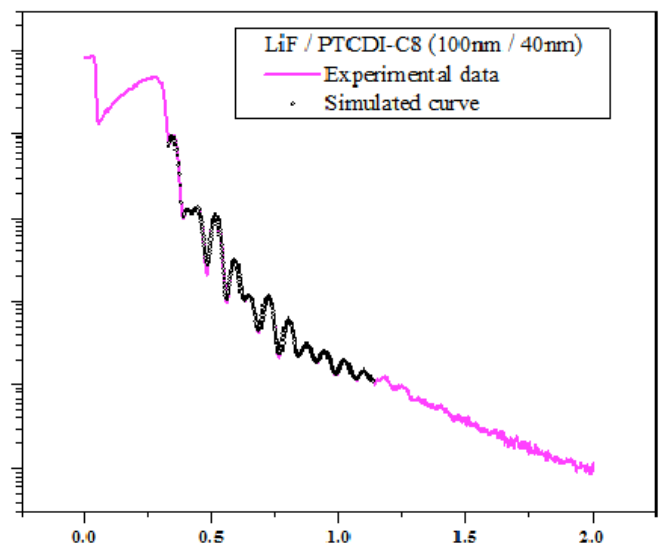

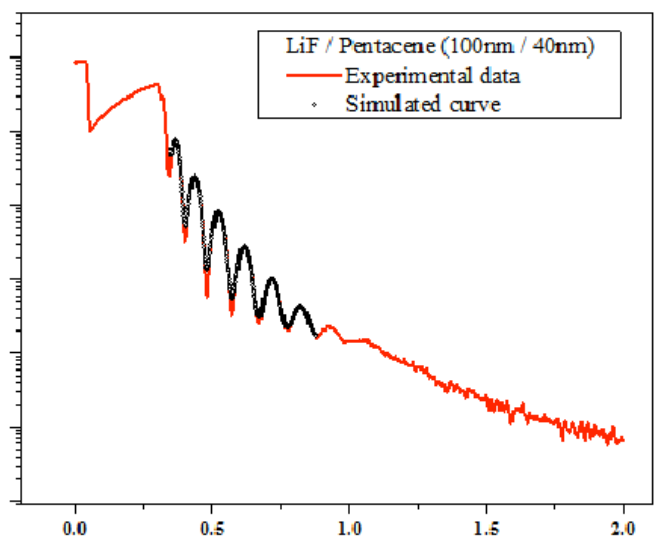

Figure 8.30 - Measured and fitted X-ray data for LiF/PTCDI-C8 and LiF/pentacene bilayers. 
OTFTs with the LiF/PTCDI-C8 and $\mathrm{LiF} /$ pentacene bilayers as the dielectric/organic layers. Ambiolar characteristics were observed for the fabricated transistors due to the effect of the LiF gate dielectric. As can be seen in Figure 8.31, devices with LiF/PTCDI-C8 bilayer exhibit about one order of magnitude higher output current $\left(\mathrm{I}_{\mathrm{ds}}\right)$ at a constant drain-source voltage $\left(\mathrm{V}_{\mathrm{ds}}\right)$ compared to the devices with $\mathrm{LiF} /$ pentacene bilayer. The observed differences in the electrical characteristics of these devices can be attributed to the effects of the dielectric/organic interface and the molecular structure of the organic layers. As confirmed from the XRR analysis, PTCDIC8 thin films have better molecular packing in the LiF/PICDI-C8 bilayer structure, which in turn leads to superior electrical characteristics for PTCDI-C8 OTFTs. 

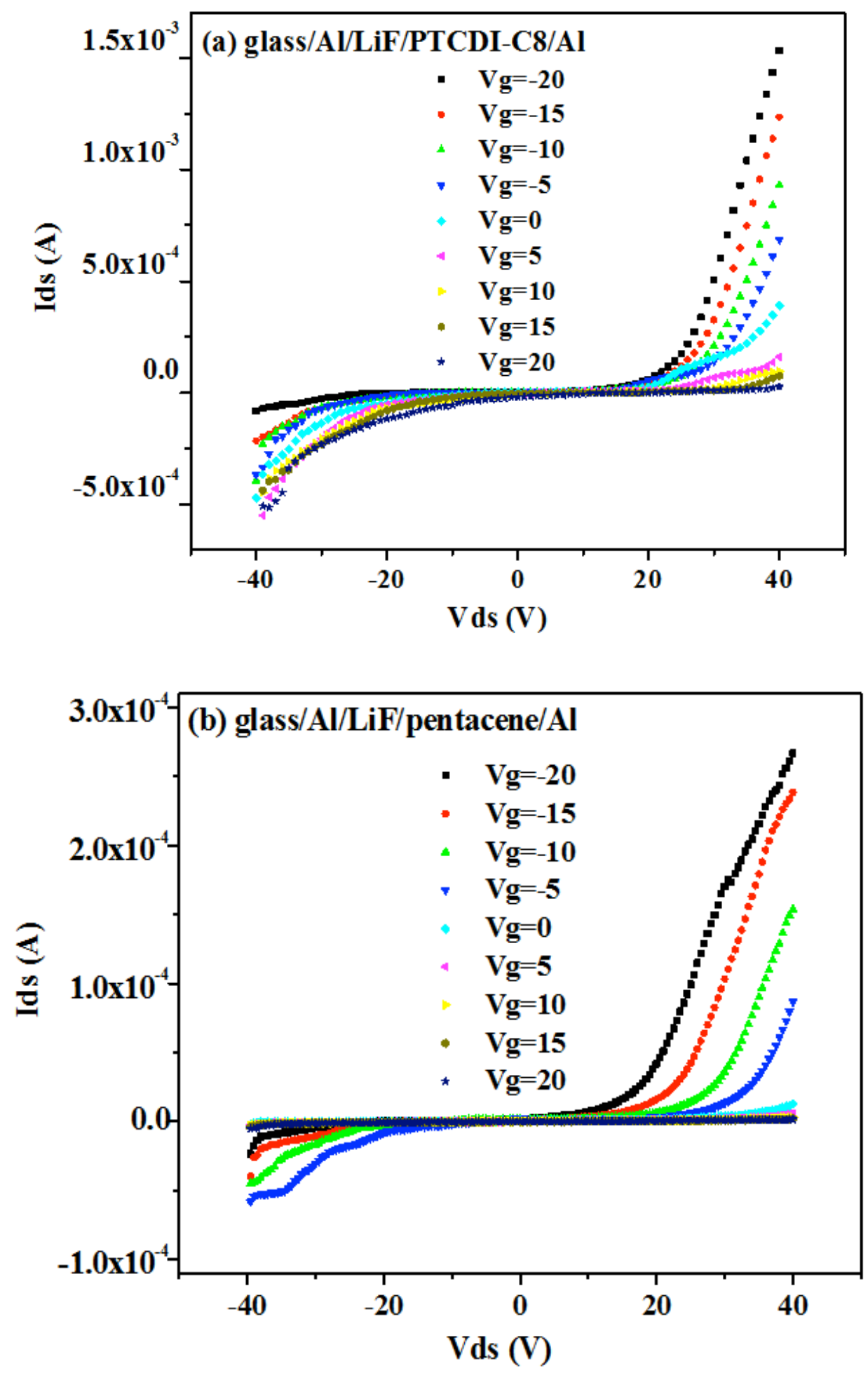

Figure 8.31 - Output characteristics of the organic thin film transistors based on (a) LiF/PTCDI$\mathrm{C} 8$ and (b) $\mathrm{LiF} /$ pentacene bilayers. 


\section{CHAPTER 9: CONCLUSIONS AND FUTURE DIRECTIONS}

In this study, several organic materials were deposited using vacuum thermal evaporation technique. The focus of this work has been on PTCDI-C8 thin films which have been shown to be a promising n-type organic material. Films with different thicknesses were prepared and their morphology and molecular structures were studied using different material characterization techniques such as X-ray reflectivity, AFM and ellipsometry. It has been shown that X-ray reflectivity is a powerful tool to study the structure of organic materials in detail. Thickness as well as surface and interface roughness can be derived from analyzing the $\mathrm{X}$-ray reflectivity measurements. Films studied in this work were shown to possess a smooth surface and a polycrystalline structure. Additionally, the effect of the substrate morphology on the film morphology were studied by preparing and characterizing organic thin films deposited on different type of substrates.

Organic solar cells with various structures of active layers such as bilayer, layered and co-deposited were fabricated. CuPc and PTCDI-C8 were used as the donor and the acceptor layers, respectively. $\mathrm{MoO}_{3}$ was used as a hole transport layer (HTL) and $\mathrm{Alq}_{3}$ was the electron transport layer (ETL). The effects of thickness variation of active layers, HTL and ETL on the device performance were studied. By increasing HTL thickness from 10 to $25 \mathrm{~nm}$, performance of the fabricated organic solar cell increases significantly. On the other hand, for thicker buffer layers, an S-shape kink has been observed in the J-V curves, which in turn reduces the efficiency of the fabricated devices significantly. The results of these studies can be used to understand and optimize organic thin film device performance. 
Moreover, organic thin film transistors based on LiF as the dielectric layer and PTCDIC8 or pentacene as the active layer were fabricated. The effects of the dielectric layer and its interface with organic layer on the electrical characteristics of the device were studied. Charge transport mechanisms in these devices were explained based on hopping and Fowler-Nordheim tunneling models. The results of this study emphasize the importance of the effects of the structural properties of organic materials as well as the device structure on the optical and electrical characteristics of organic electronic devices. Therefore, in order to enhance the performance of these devices, further detailed studies of the structure of the organic materials and charge transport mechanisms are warranted.

As for future directions, further exploration of device design, structures and parameters can be performed by utilizing new organic materials, utilizing different combinations of active and buffer layers, introducing new transparent/semi-transparent electrodes such as $\mathrm{ZnO}$, graphene and metal nano-wires instead of ITO, studying inorganic-organic hybrid structures utilizing quantum dots or photonic crystals as well as modeling the electrical and optical characteristics of various organic electronic devices via simulation. 


\section{REFERENCES}

1. M.S. Meruvia, I. A. Hümmelgen, "Hybrid molecular-inorganic semiconductor in vertical architectures”, Advanced Functional Materials, v 16,n 4, p 459-467, (2006)

2. S. Liu, W. M. Wang, A. L. Briseno, S. C. B. Mannsfeld, and Z. Bao, "Controlled deposition of crystalline organic semiconductors for field-effect-transistor applications”, Adv. Mater. 21, $1217(2009)$

3. M. E. Gershenson and V. Podzorov, A. F. Morpurgo, "Colloquium- electronic transport in single-crystal organic transistors", Rev. Mod. Phys. 78, 973 (2006).

4. C. D. Dimitrakopoulos, D. J. Mascaro "Organic thin-film transistors-a review of recent advances”, IBM J. Res. \& Dev. 45, 11 (2001)

5. P. R. L. Malenfant, C. D. Dimitrakopoulos, J. D. Gelorme, L. L. Kosbar, T. O. Graham, A. Curioni and W. Andreoni, "N-type organic thin film transistor with high field-effect mobility based on a N,N'-dialkyl-3,4,9,10-perylene tetracarboxylic diimide derivative", Appl. Phys. Lett. 80, 2517 (2002).

6. R. J. Chesterfield, J. C. McKeen, C. R. ewman, P. C. Ewbank, D. A. da Silva Filho, J.-L. Brédas, L. L. Miller, K. R. Mann, and C. D. Frisbie, "Organic Thin Film Transistors Based on N-Alkyl Perylene Diimides- Charge Transport Kinetics as a Function of Gate Voltage and Temperature", J. Phys. Chem. B 108, 19281 (2004)

7. Y. Tanabe, Macro-molecular Science and Engineering, New Aspects, Springer: New York, U.S., 319 (1999). 
8. André Moliton, Roger C.Hiorns, "Review of electronic and optical properties of semiconducting $\pi$-conjugated polymers- Applications in optoelectronics”, Polymer International, v 53, n 10, p 1397-1412 (2004)

9. I. Stenger, A. Frigout, D. Tondelier, B. Geffroy, R. Ossikovski, Y. Bonnassieux, "Polarized micro-Raman spectroscopy study of pentacene thin films", Applied Physics Letters, v 94, n 13 (2009)

10. Horng-Long Cheng, Xin-Wei Liang, Wei-Yang Chou, Yu-Shen Mai, Chou-Yu Yang, LiRen Chang, Fu-Ching Tang, "Raman spectroscopy applied to reveal polycrystalline grain structures and carrier transport properties of organic semiconductor films- application to pentacene-based organic transistors”, Organic Electronics: physics, materials, applications, v 10, n 2, p 289-298 (2009)

11. Andrew J. Lovinger, D.D. Davis, A. Dodabalapur, H.E. Katz, L. Torsi, "Single-crystal and polycrystalline morphology of the thiophene-based semiconductor $\alpha$-hexathienyl ( $\alpha-6 \mathrm{~T})$ ", Macromolecules, v 29, n 14, p $4952-4957$ (1996)

12. Cheng Horng-Long, Mai Yu-Shen, Chou Wei-Yang, Chang Li-Ren, Liang Xin-Wei, “Thickness-Dependent Structural Evolutions and Growth Models in Relation to Carrier Transport Properties in Polycrystalline Pentacene Thin Films”, Advanced Functional Materials, v 17, n 17, p 3639-49 (2007)

13. G.A. de Wijs, C.C. Mattheus, R.A. de Groot, T.T.M. Palstra, “Anisotropy of the mobility of pentacene from frustration”, Synthetic Metals, v 139, n 1, p 109-14 (2003)

14. C.C. Mattheus, A.B. Dros, J. Baas, G.T. Oostergetel, A. Meetsma, J.L. de Boer, T.T.M. Palstra, "Identification of polymorphs of pentacene", Synthetic Metals, v 138, n 3, p 475 $81(2003)$ 
15. Ingrid Stenger, Alexandre Frigout, Denis Tondelier, Bernard Geffroy, Razvigor Ossikovski, Yvan Bonnassieux, "Polarized micro-Raman spectroscopy study of pentacene thin films", Applied Physics Letters, v 94, n 13 (2009)

16. Lawrence F. Drummy, Christian Kübel, David C. Martin, "Molecular vacancies in herringbone crystals", Philosophical Magazine, v 84, n 19, p 1955-1968 (2004)

17. Zhenan Bao, Andrew J. Lovinger, Ananth Dodabalapur, "Organic field-effect transistors with high mobility based on copper phthalocyanine”, Applied Physics Letters, v 69, n 20, p $3066(1996)$

18. R. Zeis, T. Siegrist, and Ch. Kloc, "Single-crystal field-effect transistors based on copper phthalocyanine", Applied Physics Letters, v 86, n 2 (2005)

19. S. Uchida, J. Xue, B. P. Rand, and S. R. Forrest, "Organic small molecule solar cells with a homogeneously mixed copper phthalocyanine-C60 active layer”, Applied Physics Letters, v 84, n 21, (2004)

20. S. Heutz, P. Sullivan, B.M. Sanderson, S.M. Schultes, T.S. Jones, "Influence of molecular architecture and intermixing on the photovoltaic morphological and spectroscopic properties of CuPc-C60 heterojunctions", Solar Energy Materials and Solar Cells, v 83, n 2-3, p 229$245(2004)$

21. Akitaka Hoshino, Yoshiko Takenaka, Hideki Miyaji, "Redetermination of the crystal structure of $\alpha$-copper phthalocyanine grown on KCl”, Crystallographica Section B: Structural Science, v 59, n 3 (2003)

22. Christelle Vergnat, Virginie Landais, Jean-François Legrand, Martin Brinkmann, "Orienting semiconducting nanocrystals on nanostructured polycarbonate substrates- Impact of substrate 
temperature on polymorphism and in-plane orientation”, Macromolecules, v 44, n 10, p 3817-3827 (2011)

23. Daocheng Xia, Shukun Yu, Rensheng Shen, Chunyu Ma, Chuanhui Cheng, Dongmei Ji, Zhaoqi Fan, Xu Wang, Guotong Du, “A novel method for the direct synthesis of crystals of copper phthalocyanine”, Dyes and Pigments, v 78, n 1(2008)

24. O. Berger, W.-J. Fischer, B. Adolphi, S. Tierbach, V. Melev, J. Schreiber, "Studies on phase transformations of $\mathrm{Cu}$ phthalocyanine thin films", Journal of Materials Science: Materials in Electronics, v 11, n 4 (2000)

25. S. Karak, V. S. Reddy, S. K. Ray, A. Dhar, "Organic photovoltaic devices based on pentacene-N,N-dioctyl-3,4,9,10-perylenedicarboximide heterojunctions”, Organic Electronics: physics, materials, applications, v 10, n 5 (2009)

26. Shuhei Tatemichi, Musubu Ichikawa, Shimpei Kato, Toshiki Koyama, Yoshio Taniguchi, “Low-voltage, high-gain, and high-mobility organic complementary inverters based on N,N ditridecyl-3,4,9,10-perylenetetracarboxylic diimide and pentacene”, Physica Status Solidi Rapid Research Letters, v 2, n 2, (2008)

27. Joon Hak Oh, Shuhong Liu, Zhenan Bao, Rüdiger Schmidt, Frank Würthner, "Air-stable nchannel organic thin-film transistors with high field-effect mobility based on N,N'-bis (heptafluorobutyl) -3,4:9,10-perylene diimide”, Applied Physics Letters, v 91, n 21 (2007)

28. R. Thomas Weitz, Konstantin Amsharov, Ute Zschieschang, Esther Barrena Villas, Dipak K. Goswami, Marko Burghard, Helmut Dosch, Martin Jansen, Klaus Kern, Hagen Klauk, “Organic n-channel transistors based on core-cyanated perylene carboxylic diimide derivatives", Journal of the American Chemical Society, v 130, n 14 (2008) 
29. Reid J. Chesterfield, John C. McKeen, Christopher R. Newman, Paul C. Ewbank, Filho Da Silva, A. Demétrio, Jean-Luc Brédas, Larry L. Miller, Kent R. Mann, Daniel C. Frisbie, "Organic thin film transistors based on $\mathrm{N}$-alkyl perylene diimides- Charge transport kinetics as a function of gate voltage and temperature", Journal of Physical Chemistry B, v 108, n 50 (2004)

30. Patrick R.L. Malenfant, Christos D. Dimitrakopoulos, Jeffrey D. Gelorme, Laura L. Kosbar, Teresita O. Graham, Alessandro Curioni, Wanda Andreoni, "N-type organic thin-film transistor with high field-effect mobility based on a N,N prime -dialkyl-3,4,9,10-perylene tetracarboxylic diimide derivative", Applied Physics Letters, v 80, n 14 (2002).

31. Tobias N Krauss, Esther Barrena, Xue N. Zhang, Dimas G. De Oteyza, János Major, Volker Dehm, Frank Würthner, Leide P. Cavalcanti, Helmut Dosch, “Three-dimensional molecular packing of thin organic films of PTCDI determined by surface X-ray diffraction”, Langmuir, v 24, n $22(2008)$

32. Jana Zaumseil, Henning Sirringhaus, "Electron and ambipolar transport in organic fieldeffect transistors", Chemical Reviews, v 107, n 4 (2007)

33. M.C. Hamilton, J. Kanicki, “Organic polymer thin-film transistor photosensors”, IEEE Journal of Selected Topics in Quantum Electronics, v 10, n 4, p 840-8 (2004)

34. Gavin Conibeer, "Third-generation photovoltaics", Materials today, v. 10, n. 11, (2007)

35. S.O. Kasap, Optoelectronics and photonics-Principles and practices, Printice-Hall, Inc. (2001)

36. A. Rohatgi, D.S. Kim, K. Nakayashiki, V. Yelundur, B. Rounsaville, "High-efficiency solar cells on edge-defined film-fed grown (18.2\%) and string ribbon (17.8\%) silicon by rapid thermal processing", Applied Physics Letters, v 84, n 1 (2004) 
37. C. W. Tang, “Two-layer organic photovoltaic cell “, Applied Physics Letters, v 48, n 18 (1986)

38. www.nrel.gov

39. D. Wynands, M.Levichkova, M.Riede, M. Pfeiffer, P.Baeuerle, R.Rentenberger, P.Denner, K. Leo, "Correlation between morphology and performance of low bandgap oligothiopheneC60 mixed heterojunctions in organic solar cells", Journal of Applied Physics, v 107, n 1 (2010).

40. Yang Fan, M.Shtein,S.R. Forrest, "Morphology control and material mixing by hightemperature organic vapor-phase deposition and its application to thin-film solar cells", Journal of Applied Physics, v 98, n 1 (2005).

41. R. Schueppel, K. Schmidt, C.Uhrich, K.Schulze, D.Wynands, J.L.Bredas, E.Brier, E.Reinold, H.-B.Bu, P.Baeuerle, B.Maennig, M.Pfeiffer, K. Leo, “Optimizing organic photovoltaics using tailored heterojunctions-a photoinduced absorption study of oligothiophenes with low band gaps “, Physical Review B (Condensed Matter and Materials Physics), v 77, n 8 (2008).

42. R. Mondal, H.A. Becerril, E. Verploegen, Kim Dongwook, J.E. Norton, N. Miyaki, Lee Sangjun, M.F. Toney, J.-L. Bre'das, M.D. McGehee, Zhenan Bao, “Thiophene-rich fusedaromatic thienopyrazine acceptor for donor-acceptor low band-gap polymers for OTFT and polymer solar cell applications “, Journal of Materials Chemistry, v 20, n 28, (2010).

43. Srinivas Sista, Mi-Hyae Park, Ziruo Hong, Yue Wu, Jianhui Hou, Wei Lek Kwan, Gang Li, Yang Yang, "Highly efficient tandem polymer photovoltaic cells", Advanced Materials, v 22, n 3 (2010). 
44. R. Timmreck, S.O thof, K. Leo, M.K. Riede, "Highly doped layers as efficient electron-hole recombination contacts for tandem organic solar cells", Journal of Applied Physics, v 108, n $3,(2010)$.

45. G.D. Sharma, "Solution processable organic polymers and small molecules for bulkheterojunction solar cells: A review “, AIP Conference Proceedings, v 1391, (2011)

46. http://www.plusplasticelectronics.com/energy/solarmer-organic-solar-cell-efficiency-nears-85-percent-43149.aspx

47. http://www.konarka.com/index.php/site/pressreleasedetail/konarka_technologies_advances_a ward_winning_power_plastic_solar_cell_effici

48. A. Moliton, W. Rammal, B. Lucas, "How to model the behaviour of organic photovoltaic cells", EPJ Applied Physics, v 33, n 3 (2006)

49. Christoph Brabec, Ullrich Scherf, Vladimir Dyakonov, Organic Photovoltaics: Materials, Device Physics, and Manufacturing Technologies, Wiley-VCH, (2008)

50. Jenny Nelson, The Physics of Solar Cells, Imperial College, UK, (2003)

51. P. Peumans, A. Yakimov, S. R. Forrest, "Small molecular weight organic thin-film photodetectors and solar cells “,Journal of Applied Physics, v 93, n 7 (2003)

52. P. A. van Hal, R. A. J. Janssen, G. Lanzani, G. Cerullo,M. Zavelani-Rossi, and S. D. Silvestri, "Full temporal resolution of the two-step photoinduced energy-electron transfer in a fullerene-oligothiophene-fullerene triad using sub-10 fs pump-probe spectroscopy”, Chem. Phys. Lett., vol. 345 (2001)

53. P. Peumans, S. Uchida, and S. Forrest, "Efficient bulk heterojunction photovoltaic cells using small-molecular-weight organic thin films “, Nature, vol. 425 (2003) 
54. S.-B. Rim and P. Peumans, "The effects of optical interference on exciton diffusion length measurements using photocurrent spectroscopy “, J. Appl.Phys., vol. 103,n 12 (2008)

55. J. J. M. Halls, C. A. Walsh, N. C. Greenham, E. A. Marseglia, R. H. Friend, S. C. Moratti, and A. B. Holmes, "Efficient photodiodes from interpenetrating polymer networks ", Nature, vol. 376, n 6540 (1995)

56. G. Yu, J. Gao, J. C. Hummelen, F. Wudl, and A. J. Heeger, "Polymer photovoltaic cells: enhanced efficiencies via a network of internal donor-acceptor heterojunctions “, Science, vol. 270, n 5243 (1995)

57. C.J. Brabec, C. Winder, N. S. Sariciftci, J. C. Hummelen, A. Dhanabalan, P. A. Van Hal, R.A.J. Janssen, “A low-bandgap semiconducting polymer for photovoltaic devices and infrared emitting diodes “, Adv. Funct. Mater., v 12, n 10 (2002)

58. M. A. Green, K. Emery, Y. Hishikawa and W. Warta, "Solar cell efficiency tables", Progress in Photovoltaics: Research and Applications, vol. 17, n 1 (2009)

59. Bernard Kippelen, Jean-Luc Brédas, "Organic photovoltaics “, Energy and Environmental Science, v 2, n 3 (2009)

60. G. Dennler, M. C. Scharber, and C. J. Brabec, "Polymer-fullerene bulk-heterojunction solar cells", Adv. Mater., vol. 21, n 130 (2009)

61. C. Mueller, T. A. M. Ferenczi, M. Campoy-Quiles, J. M. Frost, D. D. C. Bradley, P. Smith, N. Stingelin-Stutzmann, and J. Nelson, "Binary organic photovoltaic blends- A simple rationale for optimum compositions", Adv. Mater., vol. 20, n 18 (2008)

62. G. Hadziioannou and P.F. Van Hutten, Semiconducting Polymers - Chemistry, Physics and Engineering, WILEY-VCH, U.S., 25 (2000) 
63. J. K. Rath, H. Meiling, R. E. I. Schropp, "Low-temperature deposition of polycrystalline silicon thin films by hot-wire CVD”, Solar Energy Materials and Solar Cells, v 48, n 1 (1997)

64. B. Stannowski, R. E. I. Schropp, "Hot-wire amorphous silicon thin-film transistors on glass “, Thin Solid Films, v 383, n 1-2, p 125-8 (2001)

65. C.H. Ahn, J. M. Triscone, J. Mannhart, "Electric field effect in correlated oxide systems", Nature, v 424, n 6952 (2003)

66. F. Garnier, "Thin-film transistors based on organic conjugated semiconductors", Chemical Physics, v 227, n 1-2 (1998)

67. Chu Chih-Wei, Li Sheng-Han , Chen Chieh-Wei, V. Shrotriya, Yang Yang," Highperformance organic thin-film transistors with metal oxide/metal bilayer electrode", Applied Physics Letters, v 87, n 19 (2005)

68. Claudia N. Hoth, Stelios A. Choulis, Pavel Schilinsky, Christoph J. Brabec, "High photovoltaic performance of inkjet printed polymer-Fullerene blend", Advanced Materials, v 19, n $22(2007)$

69. www.zurich.ibm.com/news/09/pentacene.htm

70. M.T. Bray, Samuel H. Cohen, Marcia L. Lightbody, Atomic Force Microscopy/Scanning Tunneling Microscopy, New York : Kluwer Academic/Plenum Publishers, (1999)

71. www.science.siu.edu/chemistry/zang/image/AFM-force.JPG

72. www.mf.mpg.de/en/abteilungen/dosch/barrena/spm-principle_en.shtml

73. Vaclav Holy, T. Baumbach, U. Pietsch, High-Resolution X-ray scattering from thin films and multilayers, Springer, (1999) 
74. Jens Als-Nielsen and Des McMorrow, Elements of Modern X-ray Physics, New York : Wiley, (1992)

75. B. E. Warren, X-ray diffraction, Courier Dover Publications, (1990)

76. L. G. Parratt, "Surface Studies of Solids by Total Reflection of X-Rays", Physical Review, v95, n2 (1954)

77. J.O. Osso, F. Schreiber, V. Kruppa, H. Dosch, M. Garriga, M.I. Alonso, F. Cerdeira, "Controlled molecular alignment in phthalocyanine thin films on stepped sapphire surfaces ", Adv. Funct. Mater. 12 (2002)

78. Harald Hoppe, Niyazi Serdar Sariciftc, "Organic solar cells: An overview”, Journal of Materials Research, v 19, n 7 (2004).

79. J.C. Wang, X.C. Ren, S.Q. Shi, C.W. Leung, Paddy K.L. Chan, “Charge accumulation induced S-shape J-V curves in bilayer heterojunction organic solar cells “, Organic Electronics: physics, materials, applications, v 12, n 6 (2011)

80. P. Peumans, S. R. Forrest, "Very-high-efficiency double-heterostructure copper phthalocyanine/C60 photovoltaic cells “, Applied Physics Letters, v 80, n 2 (2002)

81. Ankit Kumar, Srinivas Sista, Yang Yang, "Dipole induced anomalous S-shape I-V curves in polymer solar cells “, Journal of Applied Physics, v 105, n 9 (2009)

82. Wolfgang Tress, Karl Leo, Moritz Riede, "Influence of hole-transport layers and donor materials on open-circuit voltage and shape of I-V curves of organic solar cells “, Advanced Functional Materials, v 21, n 11 (2011)

83. Jin Young Kim, Sun Hee Kim, Hyun-Ho Lee, Kwanghee Lee, Wanli Ma, Xiong Gong, Alan J. Heeger, "New architecture for high-efficiency polymer photovoltaic cells using solution-based titanium oxide as an optical spacer “, Advanced Materials, v 18, n 5 (2006) 
84. Z.R. Hong, Z.H. Huang, X.T. Zeng, "Utilization of copper phthalocyanine and bathocuproine as an electron transport layer in photovoltaic cells with copper phthalocyanine/buckminsterfullerene heterojunctions: Thickness effects on photovoltaic performances “, Thin Solid Films, v 515, n 5 (2007)

85. Cheng Fei, Fang Guojia, Fan Xi, Liu Nishuang, Sun Nanhai Qin Pingli, Zheng Qiao, Wan Jiawei, Zhao Xingzhong, "Enhancing the short-circuit current and efficiency of organic solar cells using $\mathrm{MoO}_{3}$ and $\mathrm{CuPc}$ as buffer layers “, Solar Energy Materials and Solar Cells, v 95, n $10(2011)$

86. Nana Wang, Junsheng Yu, Yue Zang, Jiang Huang, Yadong Jiang, "Effect of buffer layers on the performance of organic photovoltaic cells based on copper phthalocyanine and C60 ", Solar Energy Materials and Solar Cells, v 94, n 2 (2010)

87. Shuang-hong Wu, Wen-lian Li, Bei Chu, Zi-sheng Su, Feng Zhang, C.S. Lee, "High performance small molecule photodetector with broad spectral response range from 200 to 900 nm “, Applied Physics Letters, v 99, n 2 (2011)

88. H. M. Ng, D. Doppalapudi, E. Iliopoulos, and T. D. Moustakas. "Distributed Bragg reflectors based on AlN/GaN multilayers “, Appl. Phys. Lett. 74, 1036 (1999)

89. H. A. Macleod, “Thin Film Optical Filters, Second Edition,” McGraw-Hill, (1989)

90. M. Dolores Perez, C. Borek, S.R. Forrest, M. E. Thompson, "Molecular and morphological influences on the open circuit voltages of organic photovoltaic devices", Journal of American Chemical Society, v131, n26 (2009)

91. A. Opitz, J. Wagner, W. Brutting, I. Salzmann, N. Koch, J. Manara, J. Pflaum, A. Hinderhofer, F. Schreiber, “Charge Separation at Molecular Donor-Acceptor Interfaces: 
Correlation Between Morphology and Solar Cell Performance “, IEEE Journal of Selected Topics in Quantum Electronics, v 16, n 6 (2010)

92. C. Rost, S. Karg, W. Riess, M. A. Loi, M. Murgia, and M. Muccini, “Ambipolar lightemitting organic field-effect transistor”, Appl. Phys. Lett, v 85, n 9, p 1613-15 (2004)

93. H. Yan, T. Kagata, and H. Okuzaki, “Ambipolar pentacene/C60-based field-effect transistors with high hole and electron mobilities in ambient atmosphere", Appl. Phys. Lett, v 94, p 23305 (2009)

94. J. Zaumseil, R. H. Friend, and H. Sirringhaus, "Spatial control of the recombination zone in an ambipolar light-emitting organic transistor”, Nature Mater., v 5, n 69 (2006)

95. T. Yasuda, T. Goto, K. Fujita, and T. Tsutsui, “Ambipolar pentacene field-effect transistors with calcium source-drain electrodes", Appl. Phys. Lett, v 85, n11 (2004)

96. S. Noro, T. Takenobu, Y. Iwasa, H. C. Chang, S. Kitagawa, T. Akutagawa and T. Nakamura, “Ambipolar, Single-Component, Metal-Organic Thin-Film Transistors with High and Balanced Hole and Electron Mobilities”, Advanced Materials (Weinheim, Ger.) v 20 (2008)

97. S. R. Saudari, P. R. Frail, and C. R. Kagan, “Ambipolar transport in solution-deposited pentacene transistors enhanced by molecular engineering of device contacts ", Appl. Phys. Lett, v 95, n 2 (2009)

98. J.A. Letizia, J. Rivnay, A. Facchetti, R. Ratner, A. Mark, and T.J. Marks, "Variable temperature mobility analysis of $\mathrm{n}$-channel, $\mathrm{p}$-channel and ambipolar organic field-effect transistors “, Adv. Func. Mater. 20, 50 (2010)

99. A. Kabulski and D. Korakakis, "Transport mechanism in aluminum nitride-metal multilayer junctions”, Journal of Vacuum Science \& Technology B, v 27, n 6 (2009) 
100. F. Cicoira and C. Santato, “Organic Light Emitting Field Effect Transistors: Advances and Perspectives “, Adv.Func. Mat. 17, 3421 (2007)

101. E. Mizuno, M. Taniguchi and T. Kawai, "Ambipolar organic field-effect transistor using gate insulator hysteresis”, Appl. Phys. Lett. 86, 143513 (2005)

102. Lay-Lay Chua, J. Zaumsell, C. Jul-Fen, E.C.W. Ou, P.K.H. Ho, H. Sirringhaus, and R.H. Friend, "General observation of n-type field-effect behaviour in organic semiconductors “, Nature 434, 194-9 (2005)

103. M.J. Panzer, and C.D. Frisbie, "Polymer electrolyte-gated organic field-effect transistors: Low-voltage, high-current switches for organic electronics and testbeds for probing electrical transport at high charge carrier density”, Ame. Chem. Soc. V 129, n 20 (2007)

104. T. Mills, L.G. Kaake, and X.-Y. Zhu, "Polaron and ion diffusion in a poly(3hexylthiophene) thin-film transistor gated with polymer electrolyte dielectric", Appl. Phys. A: Mat. Sci. and Proc. v 95, n 1 (2009)

105. Liu Shiyong, $\mathrm{Hu}$ Wei, Zhao Yi, Hou Jingying, Ma Chunsheng, "Improving the performance of organic thin-film transistor with a doped interlayer", Microelectronics Journal, v 38, n 4-5, p 632-6 (2007)

106. Chih-Wei Chu, Chieh-Wei Chen, Shrotriya, V., Yang Yang, "High-performance organic thin-film transistors with metal oxide/metal bilayer electrode”, Applied Physics Letters, v 87, n 19, p 193508-1-3 (2005)

107. Sheng-Han Li, Zheng Xu, Guanwen Yang,; Liping Ma, Yang Yang, "Solution-processed poly(3-hexylthiophene) vertical organic transistor", Applied Physics Letters, v 93, n 21, p $213301(2008)$ 
108. L.G. Kaake Y. Zou, M.J. Panzer, C.D.Frisbie, X.-Y.Zhu, "Vibrational spectroscopy reveals electrostatic and electrochemical doping in organic thin film transistors gated with a polymer electrolyte dielectric “, Journal of the American Chemical Society, v 129, n 25, p $7824-7830$ (2007)

109. Z. Xu, S.-h. Li, L. Ma, G. Li, G. Yang, Y. Yang, “A photoelectron spectroscopy study of tunable charge injection barrier between metal/organic interface”, Applied Physics Letters, v 93, n 2, p 023302-1-3 (2008)

110. Mok Sheung Man, Yan Feng H.L.W. Chan, "Organic phototransistor based on poly(3hexylthiophene)/ TiO2 nanoparticle composite”, Applied Physics Letters, v 93, n 2, p 023310-1-3 (2008)

111. Kamol Wasapinyokul, W.I. Milne, D.P. Chu, "Photoresponse and saturation behavior of organic thin film transistors", Journal of Applied Physics, v 105, n 2 (2009) 


\section{APPENDIX A}

Refractive Indices Calculation

clear all;

close all;

\%Wavelength Range

$\mathrm{L} 1=300$; L2 = 900;

\%Lorentz Model - Refractive Index Coefficient

$\%$ Glass substrate

$\%$ Layer $1=$ ITO

$\%$ Layer2 $=\mathrm{MoO}_{3}$

ei $2=4.9306$;

$\mathrm{A} \overline{2} 1=6.721 ; \mathrm{B} 21=0.66343 ; \mathrm{E} 21=2.8789$;

$\mathrm{A} 22=1.3088 ; \mathrm{B} 22=0.59296 ; \mathrm{E} 22=2.337$;

$\%$ Layer3 $=\mathrm{CuPc}$

ei_3 $=2.91$;

$\mathrm{A} \overline{3} 1=1.4697 ; \mathrm{B} 31=0.30464 ; \mathrm{E} 31=1.7744$;

$\mathrm{A} 32=1.2063 ; \mathrm{B} 32=0.21115 ; \mathrm{E} 32=2.0191$;

\%Layer4 = PTCDI-C8

ei_4 $=4.4099$;

$\mathrm{A} \overline{4} 1=2.9622 ; \mathrm{B} 41=0.29538 ; \mathrm{E} 41=2.7375$;

$\mathrm{A} 42=0.42802 ; \mathrm{B} 42=0.23807 ; \mathrm{E} 42=2.3213$;

$\%$ Layer $5=\mathrm{Alq}_{3}$

ei_5 $=2.3725$;

$\mathrm{A} 51=6.237 ; \mathrm{B} 51=0.11 ; \mathrm{E} 51=4.5212$;

$\mathrm{A} 52=0.8842 ; \mathrm{B} 52=0.7958 ; \mathrm{E} 52=3.4358$;

$\%$ Layer6 $=\mathrm{Al}$

\%Lorentz Model - Refractive Index

nGlass $=1.5$;

for $\mathrm{L}=\mathrm{L} 1: 1: \mathrm{L} 2$

$\mathrm{E}=1240 / \mathrm{L}$;

$\mathrm{Lm}=\mathrm{L} / 1000$;

$\mathrm{x}=\mathrm{L}+1$ - L1;

$\%$ Layer0 $=\mathrm{SiO} 2$

nLayer0 $(\mathrm{x})=\operatorname{sqrt}\left(1.28604141+1.07044083 * \mathrm{Lm}^{\wedge} 2 /\left(\mathrm{Lm}^{\wedge} 2-1.00585997 \mathrm{e}-2\right)+\right.$

$\left.1.10202242 * \mathrm{Lm}^{\wedge} 2 /\left(\mathrm{Lm}^{\wedge} 2-100\right)\right)$;

$\%$ Layer $1=\mathrm{ITO}$ 


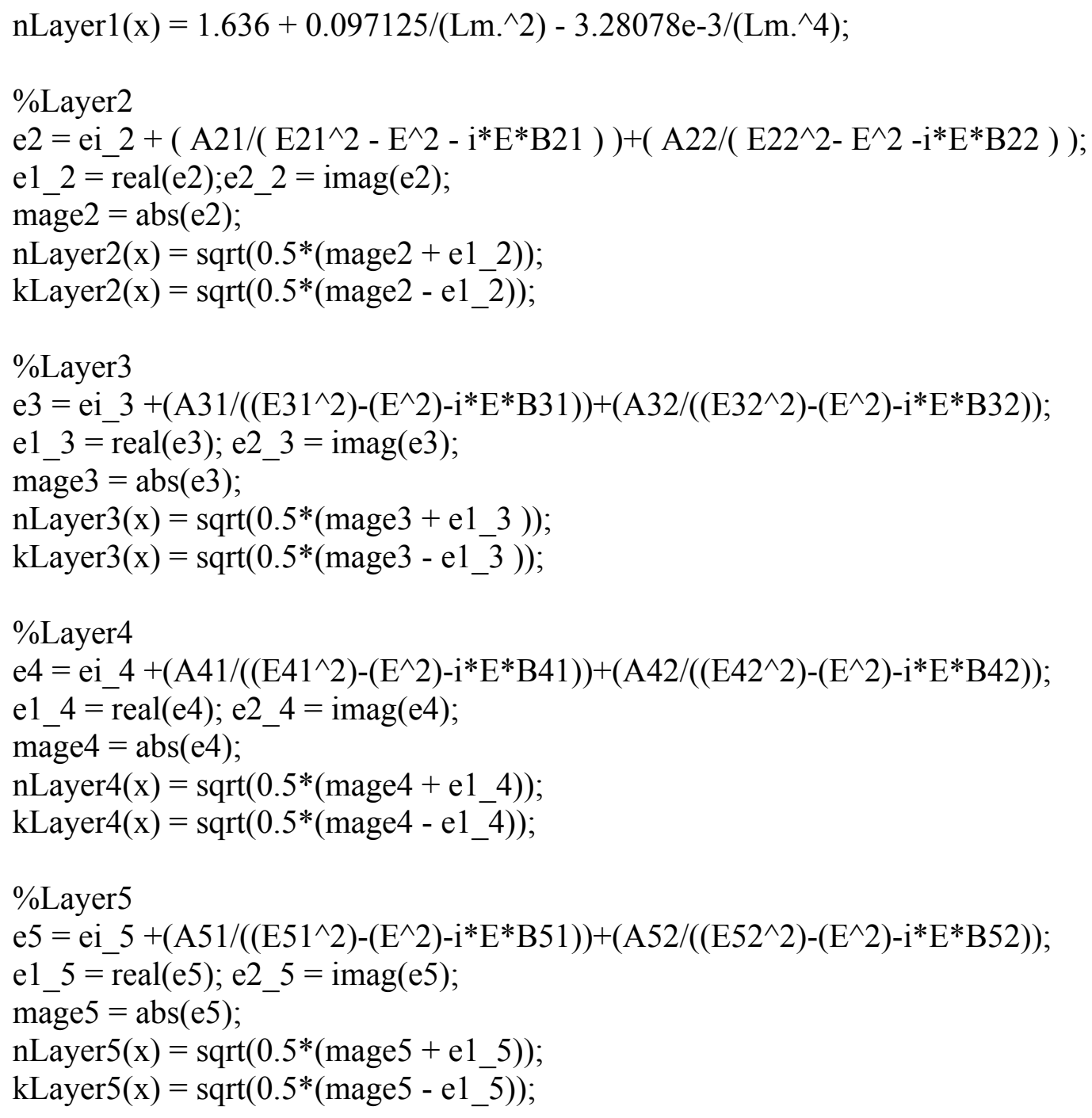

end

$\operatorname{set}\left(0\right.$, 'DefaultAxesColorOrder', $\left[\begin{array}{lll}0 & 0 & 0\end{array}\right], \ldots$

'DefaultAxesLineStyleOrder','-|-.|--|:')

subplot $(2,1,1)$;

plot(L,nLayer0,'y', L,nLayer1,'c', L,nLayer2,'r', L,nLayer3,'b',L,nLayer4,'m',L,nLayer5,'g') xlabel('Wavelenght');

ylabel('Refractive Index');

subplot $(2,1,2)$;

plot(L,kLayer2,'r', L,kLayer3,'b',L,kLayer4,'m',L,kLayer5,'g')

xlabel('Wavelenght');

ylabel('Extinction Coefficient'); 

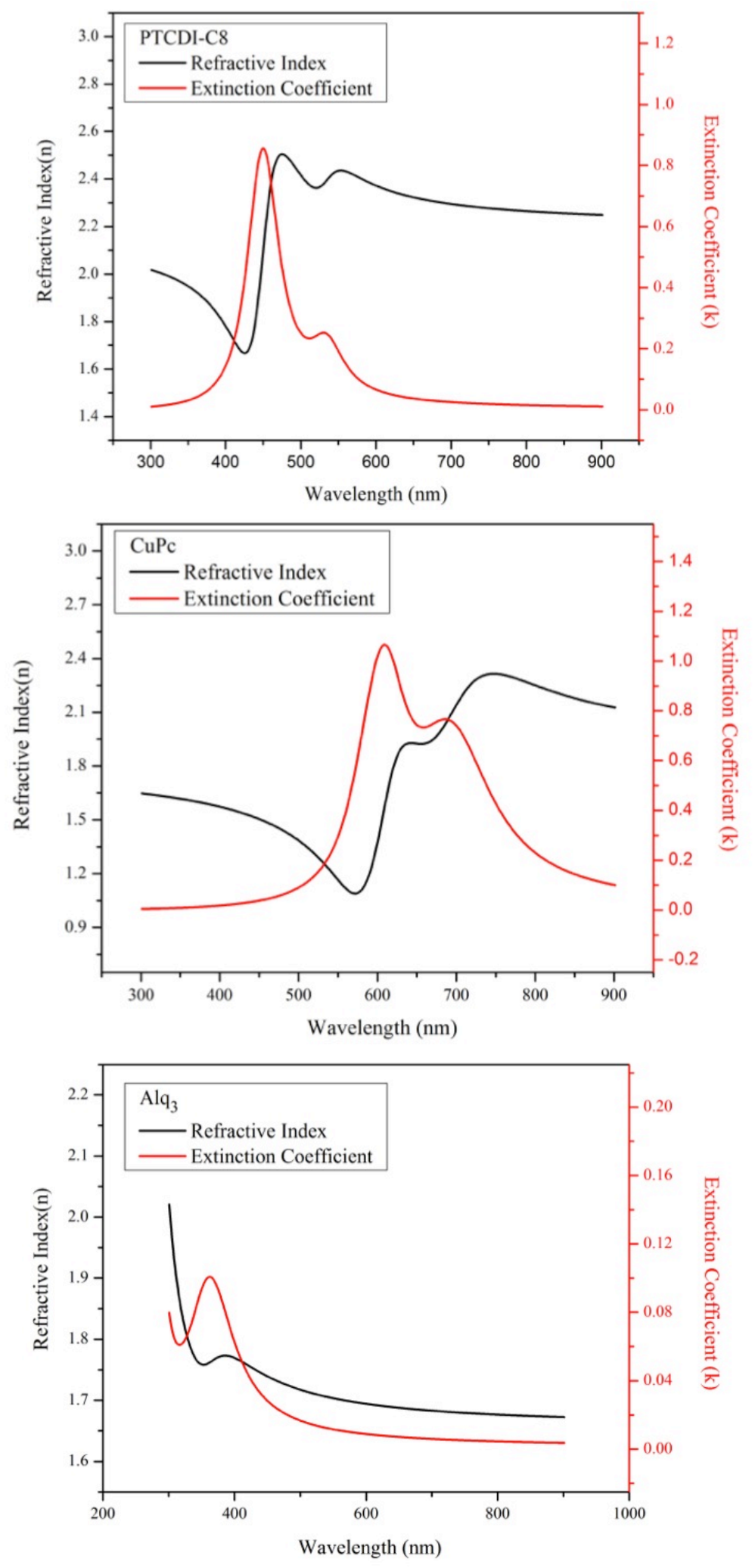

Figure A.1 -Refractive indices and extinction coefficients for CuPc, PTCDI-C8 and Alq $\mathrm{q}_{3}$ films. 


\section{APPENDIX B}

Refractivity Calculation

clear all;

close all;

\%Wavelength Range

$\mathrm{L} 1=300$; L2 = 900;

\%\&Refractive Index@500nm

nGlass $=1.5$;

$\%$ nLayer $1=1.97$

nLayer6 $=0.8$;

\%Lorentz Model - Refractive Index Coefficient

$\%$ Layer2 $=\mathrm{MoO}_{3}$

ei_2 $=4.9306$;

$\mathrm{A} 21=6.721 ; \mathrm{B} 21=0.66343 ; \mathrm{E} 21=2.8789$;

$\mathrm{A} 22=1.3088 ; \mathrm{B} 22=0.59296 ; \mathrm{E} 22=2.337$;

$\%$ Layer3 $=\mathrm{CuPc}$

ei_3 $=2.91$;

$\mathrm{A} \overline{3} 1=1.4697 ; \mathrm{B} 31=0.30464 ; \mathrm{E} 31=1.7744$;

$\mathrm{A} 32=1.2063 ; \mathrm{B} 32=0.21115 ; \mathrm{E} 32=2.0191$;

\%Layer4 = PTCDI-C8

ei_ $4=4.4099$;

$\mathrm{A} \overline{4} 1=2.9622 ; \mathrm{B} 41=0.29538 ; \mathrm{E} 41=2.7375$;

$\mathrm{A} 42=0.42802 ; \mathrm{B} 42=0.23807 ; \mathrm{E} 42=2.3213$;

$\%$ Layer5 $=\mathrm{Alq}_{3}$

ei_ $5=2.1878$;

$\mathrm{A} \overline{5} 1=6.313 ; \mathrm{B} 51=0.0328 ; \mathrm{E} 51=4.1419$;

$\mathrm{A} 52=0.67426 ; \mathrm{B} 52=0.50304 ; \mathrm{E} 52=3.1657$;

for $\mathrm{L}=\mathrm{L} 1: 1: \mathrm{L} 2$

$\mathrm{E}=1240 / \mathrm{L}$;

$\mathrm{Lm}=\mathrm{L} / 1000$

$\mathrm{x}=\mathrm{L}+1-\mathrm{L} 1$;

$\mathrm{BC}=\operatorname{eye}(2)$;

$\%$ Layer6(Al)

dLayer6 = 0 ;

n6 = nLayer6;

delta $=\left(2 *\right.$ pi $^{*}$ n6*dLayer6 $) / \mathrm{L}$;

$\mathrm{BC}=\mathrm{BC} *\left[\cos (\right.$ delta $), \mathrm{i}^{*} \sin ($ delta $) / \mathrm{n} 6 ; \mathrm{i}^{*} \mathrm{n} 6^{*} \sin ($ delta $), \cos ($ delta $\left.)\right]$; 


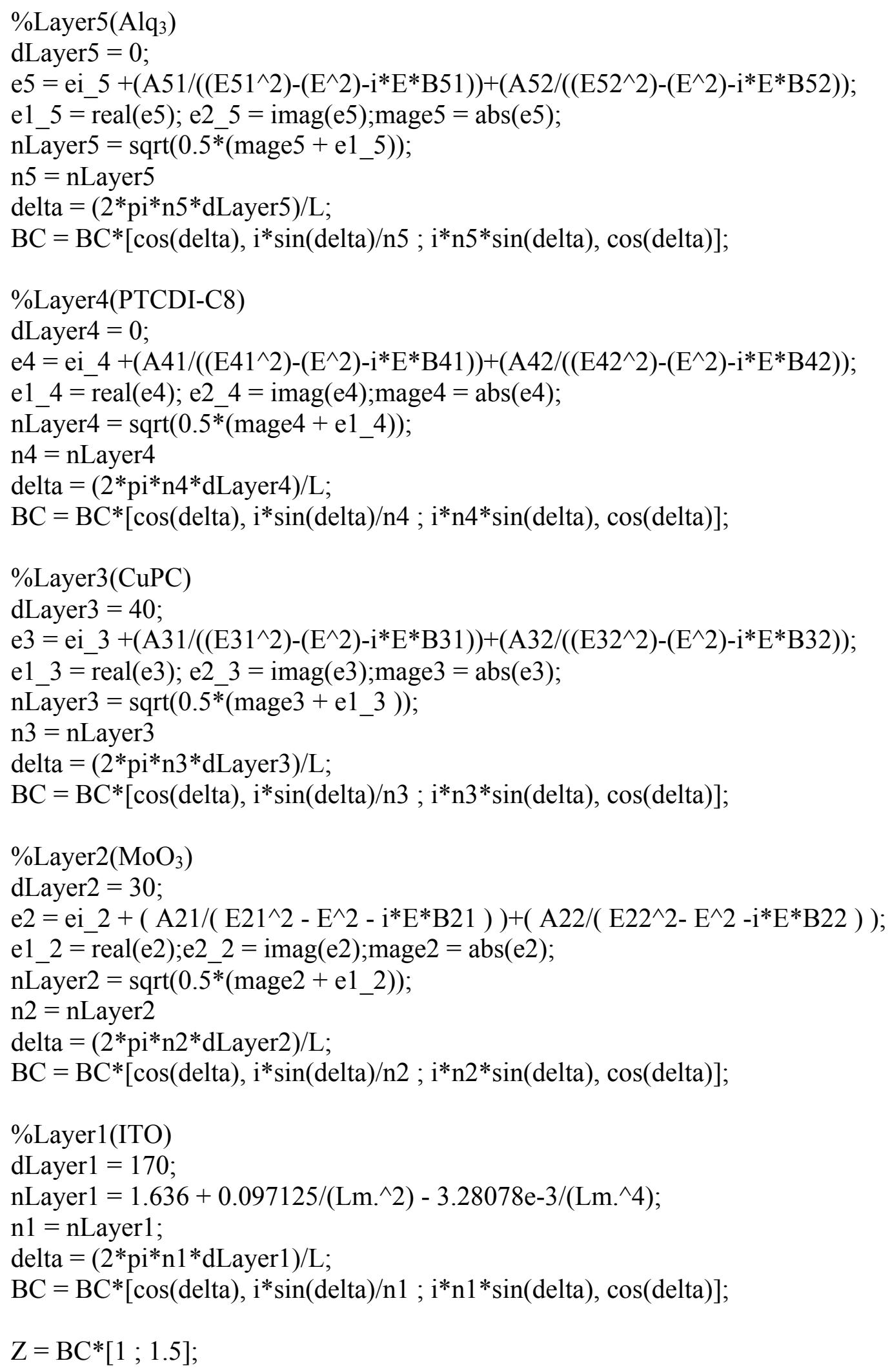


$\mathrm{Y}=\mathrm{Z}(2) / \mathrm{Z}(1)$

$\mathrm{R}(\mathrm{x})=((1-\mathrm{Y}) /(1+\mathrm{Y})) *((1-\mathrm{Y}) /(1+\mathrm{Y}))^{\prime}$;

end

$\mathrm{L}=\mathrm{L} 1: 1: \mathrm{L} 2$

$\operatorname{plot}(\mathrm{L}, \mathrm{R})$

xlabel('Wavelenght');

ylabel('Reflectivity'); 